\title{
On the exceptional set of Goldbach numbers in a short interval
}

\author{
by \\ Chao Hua Jia (Beijing)
}

1. Introduction. An even number which can be written as a sum of two primes is called a Goldbach number.

In 1937, I. M. Vinogradov proved the famous three primes theorem. Soon after that, employing Vinogradov's idea, Hua Loo Keng [3] proved that if $B$ is a sufficiently large positive constant and $N$ is sufficiently large, then the even numbers in $(2, N)$, except for $O\left(N \log ^{-B} N\right)$ values, are all Goldbach numbers.

In 1973, Ramachandra [18] obtained the result in a short interval. He showed that if $A=N^{\varphi+\varepsilon}$ with $\varphi=\frac{3}{5}$, then the even numbers in $(N, N+A)$, except for $O\left(A \log ^{-B} N\right)$ values, are all Goldbach numbers. If the estimation for the zero density of Huxley [4] is applied, $\varphi=\frac{7}{12}$ can be arrived at. In 1991, Jia Chao Hua [8] employed the sieve method combined with the circle method to get $\varphi=\frac{23}{42}$.

In 1993, Perelli and Pintz [17] developed a new technique in the circle method to make great progress in solving this problem. They showed $\varphi=\frac{7}{36}$. Mikawa [13] proved $\varphi=\frac{7}{48}$ by the sieve method.

In 1994, Jia Chao Hua [10] proved $\varphi=\frac{7}{78}$. Li Hongze [12] improved it to $\varphi=\frac{7}{81}$. Jia Chao Hua [11] got further $\varphi=\frac{1}{12}$. In [10], the new technique of [17], the sieve method and the mean value estimation for Dirichlet polynomials were applied.

In this paper, we shall develop further the technique of [10] to prove the following:

TheOREM. Suppose that $B$ is a sufficiently large positive constant, $\varepsilon$ is a sufficiently small positive constant, $N$ is sufficiently large and $A=$ $N^{\frac{7}{108}+12 \varepsilon}$. Then the even numbers in $(N, N+A)$, except for $O\left(A \log ^{-B} N\right)$ values, are all Goldbach numbers.

Project supported by the Tian Yuan Item in the National Natural Science Foundation of China. 
For $A=N^{\frac{7}{108}+12 \varepsilon}$, we can also get the conclusion of Theorem 2 of [10].

Throughout this paper, we always suppose that $B$ is a sufficiently large positive constant, $\varepsilon$ is a sufficiently small positive constant and $E=B^{2}$, $\delta=\varepsilon^{\frac{1}{3}}$. We also suppose that $N$ is sufficiently large and that $A=N^{\frac{7}{108}+12 \varepsilon}$, $Y=N^{\frac{7}{12}+\varepsilon}, Q=\frac{1}{2} \sqrt{A} \log ^{-64 B} N$. Let $c, c_{1}$ and $c_{2}$ denote positive constants which have different values at different places. $m \sim M$ means that there are positive constants $c_{1}$ and $c_{2}$ such that $c_{1} M<m \leq c_{2} M$. We often use $M(s, \chi)(M$ may be another capital letter) to denote a Dirichlet polynomial of the form

$$
M(s, \chi)=\sum_{m \sim M} \frac{a(m) \chi(m)}{m^{s}},
$$

where $a(m)$ is a complex number with $a(m)=O(1)$, and $\chi$ is a character $\bmod q$.

The author would like to thank Professors Wang Yuan and Pan Chengbiao for their encouragement.

2. Some preliminary work. Let $\chi$ be a character $\bmod q$ and let $\chi_{0}$ be a principal character. Set $E_{0}=1$ if $\chi=\chi_{0}$, and $E_{0}=0$ if $\chi \neq \chi_{0}$.

We divide the characters $\bmod q$ into two classes. We call $\chi$ a good character if $L(s, \chi)$ has no zeros in the region

$$
1-\frac{24 E \log \log Y}{\varepsilon \log Y} \leq \sigma \leq 1, \quad|t| \leq 6 Y .
$$

Otherwise, we call $\chi$ a bad character.

By Siegel's theorem and the zero free region of the $L$-function (see pp. 255 and 257 of [15]), we know that $L(s, \chi) \neq 0$ in the region

$$
\sigma>1-\frac{c(\mu)}{\max \left(q^{\mu}, \log ^{\frac{4}{5}}(|t|+2)\right)},
$$

where $\mu(>0)$ is arbitrary and $c(\mu)>0$.

Take $\mu=\varepsilon /(500 E)$. If a bad character exists, then

$$
q \geq \log \frac{300 E}{\varepsilon} N .
$$

Moreover, by the estimation for the zero density (see [14] and [5]),

$$
\sum_{\chi(\bmod q)} N(\sigma, T, \chi) \ll(q T)^{\left(\frac{12}{5}+\varepsilon\right)(1-\sigma)} \log ^{14} q T,
$$

we know that for any modulus $q \leq Q$, the number of bad characters is $O\left(\log \frac{64 E}{\varepsilon} N\right)$. Let

$$
I(a, q)=\left(\frac{a}{q}-\frac{\log ^{2 E} N}{q Y}, \frac{a}{q}+\frac{\log ^{2 E} N}{q Y}\right)
$$


and let

$$
E_{1}=\bigcup_{q \leq \log ^{E}} \bigcup_{N}^{q} I(a, q), \quad E_{2}=[1 / Q, 1+1 / Q)-E_{1} .
$$

Lemma 1. Assume that $r(\geq 2)$ and $q$ are positive integers, $d_{r}(n)=$ $\sum_{n=n_{1} \ldots n_{r}} 1$ and $x^{\varepsilon}<y \leq x$. Then

$$
\sum_{x-y<n \leq x} d_{r}^{q}(n) \ll y(\log x)^{r^{q}-1} .
$$

Proof. See Theorem 2 of [20].

Let

$$
\tau(\chi)=\sum_{r=1}^{q} \chi(r) e\left(\frac{r}{q}\right) .
$$

Lemma 2. $\tau\left(\chi_{0}\right)=\mu(q)$. For any character $\chi \bmod q,|\tau(\chi)| \leq \sqrt{q}$.

Pr o of. See pp. 24 and 28 of [15].

Lemma 3. For any complex numbers a $(n)$,

$$
\int_{-\eta}^{\eta}\left|\sum_{n} a(n) e(n t)\right|^{2} d t \ll \eta^{2} \int_{-\infty}^{\infty}\left|\sum_{x<n \leq x+1 /(2 \eta)} a(n)\right|^{2} d x .
$$

Proof. See Lemma 1 of [1].

Lemma 4. Suppose $T \geq 1, \chi$ is a character $\bmod q$, and $\sum_{m \sim M}|a(m)|^{2}$ $\ll M \log ^{c} T$. Then

$$
\sum_{\chi(\bmod q)} \int_{-T}^{T}\left|\sum_{m \sim M} \frac{a(m) \chi(m)}{m^{\frac{1}{2}+i t}}\right|^{2} d t \ll(M+q T) \log ^{c} T .
$$

Proof. See Theorem 3 on p. 38 of [15].

\section{Mean value estimate (I)}

Lemma 5. Assume that $Y^{\delta} \ll H \ll Y^{\frac{16}{135}}, M H=Y, q \leq Q$, $\chi$ is a character $\bmod q, M(s, \chi)$ is a Dirichlet polynomial and

$$
H(s, \chi)=\sum_{h \sim H} \frac{\Lambda(h) \chi(h)}{h^{s}} .
$$


Let $\eta=q Q / Y, b=1+1 / \log N$ and $T_{0}=\log ^{\frac{E}{\delta^{2}}} Y$. Then for $T_{0} \leq T \leq Y$, we have

$$
\min ^{2}\left(\eta, \frac{1}{T}\right) \sum_{\chi(\text { good })} \int_{T}^{2 T}|M(b+i t, \chi) H(b+i t, \chi)|^{2} d t \ll \eta^{2} \log ^{-6 E} N .
$$

Proof. Let $s=b+i t$ and let $\chi$ be a good character $\bmod q$. By (1) and the zero free region of the $L$-function, we know that for $|t| \leq 2 Y$,

$$
\sum_{c_{1} H<h \leq c_{2} H} \frac{\Lambda(h) \chi(h)}{h^{s}}=E_{0} \frac{\left(c_{2} H\right)^{1-s}-\left(c_{1} H\right)^{1-s}}{1-s}+O\left(\log ^{-\frac{2 E}{\delta^{2}}} Y\right) .
$$

So, for $T_{0} \leq|t| \leq 2 Y$,

$$
H(s, \chi) \ll \log ^{-\frac{E}{\delta^{2}}} Y .
$$

According to the discussion in [2], there are $O\left(\log ^{2} Y\right)$ sets $S(V, W)$, where $S(V, W)=\left\{t_{j}(\chi): \chi\right.$ runs through all good characters $\bmod q, j=$ $\left.1, \ldots, J(\chi),\left|t_{r}(\chi)-t_{s}(\chi)\right| \geq 1(r \neq s)\right\}$. For $t_{j}(\chi) \in S(V, W)$,

$$
\begin{aligned}
V & \leq M^{\frac{1}{2}}\left|M\left(b+i t_{j}(\chi), \chi\right)\right|<2 V, \\
W & \leq H^{\frac{1}{2}}\left|H\left(b+i t_{j}(\chi), \chi\right)\right|<2 W,
\end{aligned}
$$

where $Y^{-1} \leq M^{-\frac{1}{2}} V, Y^{-1} \leq H^{-\frac{1}{2}} W$ and $V \ll M^{\frac{1}{2}}, W \ll H^{\frac{1}{2}} \log ^{-\frac{E}{\delta^{2}}} Y$.

Thus

(6) $\sum_{\chi \text { (good) }} \int_{T}^{2 T}|M(b+i t, \chi) H(b+i t, \chi)|^{2} d t \ll V^{2} W^{2} Y^{-1} \log ^{2} Y|S(V, W)|$,

where $S(V, W)$ is one of the sets with the above properties.

Assume $Y^{\frac{1}{k+1}} \leq H \leq Y^{\frac{1}{k}}$, where $k$ is a positive integer, $k \geq 8$ and $k \delta \ll 1$. Applying the mean value estimate (see Theorem 3 on p. 632 of [16]) and Lemma 1 to $M(s, \chi)$ and $H^{k}(s, \chi)$, we have

$$
\begin{aligned}
& |S(V, W)| \ll V^{-2}(M+q T) \log ^{d} Y, \\
& |S(V, W)| \ll W^{-2 k}\left(H^{k}+q T\right) \log ^{d} Y,
\end{aligned}
$$

where $d=c / \delta^{2}$. Applying the Halász method (see Theorem 6 on p. 650 of [16]) to $M(s, \chi)$ and $H^{k}(s, \chi)$, we have

$$
\begin{aligned}
& |S(V, W)| \ll\left(V^{-2} M+V^{-6} q T M\right) \log ^{d} Y, \\
& |S(V, W)| \ll\left(W^{-2 k} H^{k}+W^{-6 k} q T H^{k}\right) \log ^{d} Y .
\end{aligned}
$$

Thus,

$$
V^{2} W^{2}|S(V, W)| \ll V^{2} W^{2} F \log ^{d} Y
$$


where

$$
\begin{array}{r}
F=\min \left\{V^{-2}(M+q T), V^{-2} M+V^{-6} q T M, W^{-2 k}\left(H^{k}+q T\right),\right. \\
\left.W^{-2 k} H^{k}+W^{-6 k} q T H^{k}\right\} .
\end{array}
$$

It will be proved that

$$
\min ^{2}\left(\eta, \frac{1}{T}\right) V^{2} W^{2} F \ll \eta^{2} Y \log ^{-\frac{E}{2 \delta^{2}}} N
$$

We consider four cases.

(a) $F \leq 2 V^{-2} M, 2 W^{-2 k} H^{k}$. Then

$$
\begin{aligned}
V^{2} W^{2} F & \ll V^{2} W^{2} \min \left\{V^{-2} M, W^{-2 k} H^{k}\right\} \\
& \leq V^{2} W^{2}\left(V^{-2} M\right)^{1-\frac{1}{2 k}}\left(W^{-2 k} H^{k}\right)^{\frac{1}{2 k}} \\
& =V^{\frac{1}{k}} W M^{1-\frac{1}{2 k}} H^{\frac{1}{2}} \ll Y \log ^{-\frac{E}{2 \delta^{2}}} N .
\end{aligned}
$$

(b) $F>2 V^{-2} M, 2 W^{-2 k} H^{k}$. Then

$$
\begin{aligned}
V^{2} W^{2} F & \ll V^{2} W^{2} \min \left\{V^{-2} q T, V^{-6} q T M, W^{-2 k} q T, W^{-6 k} q T H^{k}\right\} \\
& \leq V^{2} W^{2}\left(V^{-2}\right)^{1-\frac{3}{2 k}}\left(V^{-6} M\right)^{\frac{1}{2 k}}\left(W^{-2 k}\right)^{\frac{1}{k}} q T=q T M^{\frac{1}{2 k}} .
\end{aligned}
$$

Since $k \geq 8$, we have $H \geq Y^{\frac{1}{k+1}} \geq Y^{1-\frac{k}{9}}, M^{\frac{1}{2 k}} \ll Y^{\frac{1}{18}}$ and so

$$
\min ^{2}\left(\eta, \frac{1}{T}\right) V^{2} W^{2} F \ll \frac{\eta}{T} Y^{\frac{1}{18}} q T \ll \eta^{2} Y^{1-\varepsilon} .
$$

(c) $F \leq 2 V^{-2} M, F>2 W^{-2 k} H^{k}$. Then

$$
\begin{aligned}
V^{2} W^{2} F & \ll V^{2} W^{2} \min \left\{V^{-2} M, W^{-2 k} q T, W^{-6 k} q T H^{k}\right\} \\
& \leq V^{2} W^{2}\left(V^{-2} M\right)^{1-\frac{1}{3 k}}\left(W^{-6 k} q T H^{k}\right)^{\frac{1}{3 k}} \\
& \ll M H^{\frac{1}{3}}(q T)^{\frac{1}{3 k}}
\end{aligned}
$$

since $V \ll M^{\frac{1}{2}}$. As $H \geq Y^{\frac{1}{k+1}} \geq\left(\frac{Y}{Q}\right)^{\frac{1}{2 k}} Y^{2 \varepsilon}$, we have

$$
\begin{aligned}
& \min ^{2}\left(\eta, \frac{1}{T}\right) V^{2} W^{2} F \ll \eta^{2-\frac{1}{3 k}} T^{-\frac{1}{3 k}} Y\left(\frac{Y}{Q}\right)^{-\frac{1}{3 k}}(q T)^{\frac{1}{3 k}} Y^{-\varepsilon} \ll \eta^{2} Y^{1-\varepsilon} . \\
& \begin{aligned}
& \text { (d) } F>2 V^{-2} M, F \leq 2 W^{-2 k} H^{k} . \text { Then } \\
& V^{2} W^{2} F \ll V^{2} W^{2} \min \left\{V^{-2} q T, V^{-6} q T M, W^{-2 k} H^{k}\right\} \\
& \leq V^{2} W^{2}\left(V^{-2} q T\right)^{1-\frac{3}{2 k}}\left(V^{-6} q T M\right)^{\frac{1}{2 k}}\left(W^{-2 k} H^{k}\right)^{\frac{1}{k}} \\
&=(q T)^{1-\frac{1}{k}} H M^{\frac{1}{2 k}} .
\end{aligned}
\end{aligned}
$$


If $k \geq 9$, then $H \leq Y^{\frac{1}{k}} \leq Y^{1-\frac{17(k-1)}{9(2 k-1)}}, M \gg Y^{\frac{17(k-1)}{9(2 k-1)}}$, and so

$$
\min ^{2}\left(\eta, \frac{1}{T}\right) V^{2} W^{2} F \ll \eta^{2} Y\left(\frac{Y}{Q}\right)^{1-\frac{1}{k}} Y^{-\frac{17}{18}\left(1-\frac{1}{k}\right)} \ll \eta^{2} Y^{1-\varepsilon} .
$$

If $k=8$, then $Y^{\frac{1}{9}} \leq H \ll Y^{\frac{16}{135}}, M \gg Y^{\frac{119}{135}}$, and so

$$
\min ^{2}\left(\eta, \frac{1}{T}\right) V^{2} W^{2} F \ll \eta^{2} Y\left(\frac{Y}{Q}\right)^{\frac{7}{8}} Y^{-\frac{119}{144}} \ll \eta^{2} Y^{1-\varepsilon} .
$$

Combining the above, we obtain (7). Hence, Lemma 5 follows.

Lemma 6. Assume that $M \ll Y^{\frac{19}{36}}, M L=Y, q \leq Q, \chi$ is a character $\bmod q, M(s, \chi)$ is a Dirichlet polynomial, and

$$
F(s, \chi)=M(s, \chi) \sum_{l \sim L} \frac{\chi(l)}{l^{s}} .
$$

Let $\eta=q Q / Y, b=1+1 / \log N$ and $T_{1}=\sqrt{L / q}$. Then for $T_{1} \leq T \leq Y$, we have

$$
\min ^{2}\left(\eta, \frac{1}{T}\right) \sum_{\chi(\bmod q)} \int_{T}^{2 T}|F(b+i t, \chi)|^{2} d t \ll \eta^{2} Y^{-\varepsilon} .
$$

Pro of. Perron's formula yields

$$
\begin{aligned}
\sum_{l \sim L} \frac{\chi(l)}{l^{b+i t}}= & \frac{1}{2 \pi i} \int_{-4 i T}^{4 i T} L(b+i t+s, \chi) \frac{\left(c_{2} L\right)^{s}-\left(c_{1} L\right)^{s}}{s} d s \\
& +O\left(\frac{Y^{\varepsilon}}{T}\right)+O\left(\frac{1}{L}\right) \\
= & \frac{1}{2 \pi i} \int_{\frac{1}{2}-b-4 i T}^{\frac{1}{2}-b+4 i T} L(b+i t+s, \chi) \frac{\left(c_{2} L\right)^{s}-\left(c_{1} L\right)^{s}}{s} d s \\
& +O\left(\frac{Y^{\varepsilon}}{T}\right)+O\left(\frac{1}{L}\right)+O\left(\frac{L^{-\frac{1}{2}}(q T)^{\frac{1}{4}+\varepsilon}}{T}\right), \\
\left|\sum_{l \sim L} \frac{\chi(l)}{l^{b+i t}}\right|^{2} \ll & L^{-1} \log T \int_{-6 T}^{6 T}\left|L\left(\frac{1}{2}+i u, \chi\right)\right|^{2} \frac{d u}{1+|t-u|} \\
& +O\left(\frac{Y^{2 \varepsilon}}{T^{2}}\right)+O\left(\frac{1}{L^{2}}\right)+O\left(\frac{L^{-1}(q T)^{\frac{1}{2}+2 \varepsilon}}{T^{2}}\right) .
\end{aligned}
$$


By Lemma 4 and the mean value estimate for the fourth power of the $L$-function, we have

$$
\begin{aligned}
& \sum_{\chi(\bmod q)} \int_{T}^{2 T}|F(b+i t, \chi)|^{2} d t \\
& \ll L^{-1} \log N \sum_{\chi(\bmod q)} \int_{T}^{2 T}|M(b+i t, \chi)|^{2} d t\left(\int_{-6 T}^{6 T}\left|L\left(\frac{1}{2}+i u, \chi\right)\right|^{2} \frac{d u}{1+|t-u|}\right) \\
& \quad+O\left(\frac{Y^{2 \varepsilon}}{T^{2}}+\frac{1}{L^{2}}+\frac{L^{-1}(q T)^{\frac{1}{2}+2 \varepsilon}}{T^{2}}\right) \sum_{\chi(\bmod q)} \int_{T}^{2 T}|M(b+i t, \chi)|^{2} d t \\
& \ll L^{-1} \log ^{2} N\left(\sum_{\chi(\bmod q)} \int_{T}^{2 T}|M(b+i t, \chi)|^{4} d t\right)^{\frac{1}{2}} \\
& \quad \times\left(\sum_{\chi(\bmod q)}^{2 T} \int_{T}^{6 T} d t \int_{-6 T}^{6 T} \frac{\left|L\left(\frac{1}{2}+i u, \chi\right)\right|^{4}}{1+|t-u|} d u\right)^{\frac{1}{2}} \\
& \quad+O\left(\frac{Y^{2 \varepsilon}}{T^{2}}+\frac{1}{L^{2}}+\frac{L^{-1}(q T)^{\frac{1}{2}+2 \varepsilon}}{T^{2}}\right)\left(1+\frac{q T}{M}\right) \\
& \ll L^{-1}\left(1+\frac{q T}{M^{2}}\right)^{\frac{1}{2}}(q T)^{\frac{1}{2}} \log ^{10} N+Y^{-2 \varepsilon} .
\end{aligned}
$$

Hence,

$$
\begin{aligned}
\min ^{2}(\eta, & \left.\frac{1}{T}\right) \sum_{\chi(\bmod q)} \int_{T}^{2 T}|F(b+i t, \chi)|^{2} d t \\
& \ll \min ^{2}\left(\eta, \frac{1}{T}\right) Y^{-1}\left(Y^{\frac{19}{18}}+q T\right)^{\frac{1}{2}}(q T)^{\frac{1}{2}} \log ^{10} N+\eta^{2} Y^{-2 \varepsilon} \\
& \ll \eta^{2} Y^{-1}\left(Y^{\frac{19}{18}}+\frac{Y}{Q}\right)^{\frac{1}{2}}\left(\frac{Y}{Q}\right)^{\frac{1}{2}} \log ^{10} N+\eta^{2} Y^{-2 \varepsilon} \ll \eta^{2} Y^{-2 \varepsilon} .
\end{aligned}
$$

Thus Lemma 6 follows.

\section{Mean value estimate (II)}

Lemma 7. Assume that $M H K=Y, q \leq Q, \chi$ is a character $\bmod q$, $M(s, \chi), H(s, \chi)$ and $K(s, \chi)$ are Dirichlet polynomials and $G(s, \chi)=$ $M(s, \chi) H(s, \chi) K(s, \chi)$. Let $\eta=q Q / Y, b=1+1 / \log N, T_{0}=\log \frac{E}{\delta^{2}} Y$. Assume further that for $T_{0} \leq|t| \leq 2 Y, M(b+i t, \chi) \ll \log ^{-\frac{E}{\delta^{2}}} Y$ and 
$H(b+i t, \chi) \ll \log ^{-\frac{E}{\delta^{2}}} Y$. Moreover, suppose that $M$ and $H$ satisfy one of the following four conditions:

1) $M H \ll Y^{\frac{142}{261}}, Y^{\frac{17}{99}} \ll H, M^{29} / H \ll Y^{\frac{91}{9}}, Y^{\frac{17}{57}} \ll M, H^{29} / M \ll Y^{\frac{19}{3}}$, $Y^{\frac{17}{33}} \ll M^{\frac{12}{11}} H$

2) $M H \ll Y^{\frac{47}{81}}, Y^{\frac{16}{135}} \ll H, M^{\frac{29}{19}} H \ll Y^{\frac{89}{114}}, Y^{\frac{68}{81}} \ll M^{2} H, H^{4} / M \ll$ $Y^{\frac{1}{2}}, Y^{\frac{85}{147}} \ll M^{\frac{58}{49}} H$

3) $M H \ll Y^{\frac{113}{198}}, Y^{\frac{17}{90}} \ll H, M^{6} H \ll Y^{\frac{20}{9}}, Y^{\frac{17}{63}} \ll M, M^{\frac{1}{8}} H \ll Y^{\frac{7}{24}}$, $Y^{\frac{17}{30}} \ll M^{\frac{6}{5}} H$

4) $M H \ll Y^{\frac{25}{42}}, Y^{\frac{16}{135}} \ll H, M^{\frac{23}{15}} H \ll Y^{\frac{7}{9}}, Y^{\frac{17}{21}} \ll M^{2} H, H^{3} / M \ll$ $Y^{\frac{7}{18}}, Y^{\frac{68}{135}} \ll M^{\frac{14}{15}} H, Y^{\frac{5}{9}} \ll M H$.

Then for $T_{0} \leq T \leq Y$, we have

$$
\min ^{2}\left(\eta, \frac{1}{T}\right) \sum_{\chi(\text { good })} \int_{T}^{2 T}|G(b+i t, \chi)|^{2} d t \ll \eta^{2} \log ^{-6 E} N .
$$

Proof. We only show that for $T=1 / \eta=Y /(q Q)$,

$$
I=\sum_{\chi \text { (good) }} \int_{T}^{2 T}|G(b+i t, \chi)|^{2} d t \ll \log ^{-6 E} N .
$$

I. First, we assume condition 1). On applying the mean value estimate and Halász method to $M^{3}(s, \chi), H^{5}(s, \chi)$ and $K^{2}(s, \chi)$, we get

$$
I \ll U^{2} V^{2} W^{2} Y^{-1} F \log ^{c} N,
$$

where

$$
\begin{gathered}
F=\min \left\{V^{-6}\left(M^{3}+q T\right), V^{-6} M^{3}+V^{-18} q T M^{3}, W^{-10}\left(H^{5}+q T\right),\right. \\
\left.W^{-10} H^{5}+W^{-30} q T H^{5}, U^{-4}\left(K^{2}+q T\right), U^{-4} K^{2}+U^{-12} q T K^{2}\right\} .
\end{gathered}
$$

We discuss the following cases:

(a) $F \leq 2 V^{-6} M^{3}, 2 W^{-10} H^{5}, 2 U^{-4} K^{2}$. Then

$$
\begin{aligned}
U^{2} V^{2} W^{2} F & \ll U^{2} V^{2} W^{2} \min \left\{V^{-6} M^{3}, W^{-10} H^{5}, U^{-4} K^{2}\right\} \\
& \leq U^{2} V^{2} W^{2}\left(V^{-6} M^{3}\right)^{\frac{5}{16}}\left(W^{-10} H^{5}\right)^{\frac{1}{5}}\left(U^{-4} K^{2}\right)^{\frac{39}{80}} \\
& =V^{\frac{1}{8}} M^{\frac{15}{16}} U^{\frac{1}{20}} K^{\frac{39}{40}} H \ll Y \log ^{-7 E} N .
\end{aligned}
$$

(b) $F \leq 2 V^{-6} M^{3}, 2 W^{-10} H^{5}, F>2 U^{-4} K^{2}$. Then

$$
\begin{aligned}
U^{2} V^{2} W^{2} F & \ll U^{2} V^{2} W^{2} \min \left\{V^{-6} M^{3}, W^{-10} H^{5}, U^{-4} q T, U^{-12} q T K^{2}\right\} \\
& \leq U^{2} V^{2} W^{2}\left(V^{-6} M^{3}\right)^{\frac{1}{3}}\left(W^{-10} H^{5}\right)^{\frac{1}{5}}\left(U^{-4} q T\right)^{\frac{9}{20}}\left(U^{-12} q T K^{2}\right)^{\frac{1}{60}} \\
& =(q T)^{\frac{7}{15}} M H K^{\frac{1}{30}} \ll Y^{1-\varepsilon} .
\end{aligned}
$$


(c) $F \leq 2 V^{-6} M^{3}, F>2 W^{-10} H^{5}, F \leq 2 U^{-4} K^{2}$. Then

$$
\begin{aligned}
& U^{2} V^{2} W^{2} F \ll U^{2} V^{2} W^{2} \min \left\{V^{-6} M^{3}, W^{-10} q T, W^{-30} q T H^{5}, U^{-4} K^{2}\right\} \\
& \quad \leq U^{2} V^{2} W^{2}\left(V^{-6} M^{3}\right)^{\frac{1}{3}}\left(W^{-10} q T\right)^{\frac{3}{20}}\left(W^{-30} q T H^{5}\right)^{\frac{1}{60}}\left(U^{-4} K^{2}\right)^{\frac{1}{2}} \\
& \quad=(q T)^{\frac{1}{6}} M K H^{\frac{1}{12}} \ll Y^{1-\varepsilon} . \\
& \text { (d) } F \leq 2 V^{-6} M^{3}, F>2 W^{-10} H^{5}, 2 U^{-4} K^{2} \text {. Then } \\
& U^{2} V^{2} W^{2} F \\
& \ll U^{2} V^{2} W^{2} \min \left\{V^{-6} M^{3}, W^{-10} q T, W^{-30} q T H^{5}, U^{-4} q T, U^{-12} q T K^{2}\right\} \\
& \leq U^{2} V^{2} W^{2}\left(V^{-6} M^{3}\right)^{\frac{1}{3}}\left(W^{-10} q T\right)^{\frac{1}{5}}\left(U^{-4} q T\right)^{\frac{9}{20}}\left(U^{-12} q T K^{2}\right)^{\frac{1}{60}} \\
& =(q T)^{\frac{2}{3}} M K^{\frac{1}{30}} \ll Y^{1-\varepsilon} .
\end{aligned}
$$

(e) $F>2 V^{-6} M^{3}, F \leq 2 W^{-10} H^{5}, 2 U^{-4} K^{2}$. Then

$$
\begin{aligned}
U^{2} V^{2} W^{2} F & \ll U^{2} V^{2} W^{2} \min \left\{V^{-6} q T, V^{-18} q T M^{3}, W^{-10} H^{5}, U^{-4} K^{2}\right\} \\
& \leq U^{2} V^{2} W^{2}\left(V^{-6} q T\right)^{\frac{17}{60}}\left(V^{-18} q T M^{3}\right)^{\frac{1}{60}}\left(W^{-10} H^{5}\right)^{\frac{1}{5}}\left(U^{-4} K^{2}\right)^{\frac{1}{2}} \\
& =(q T)^{\frac{3}{10}} M^{\frac{1}{20}} H K \ll Y^{1-\varepsilon} .
\end{aligned}
$$

(f) $F>2 V^{-6} M^{3}, F \leq 2 W^{-10} H^{5}, F>2 U^{-4} K^{2}$. Then $U^{2} V^{2} W^{2} F$

$$
\begin{aligned}
& \ll U^{2} V^{2} W^{2} \min \left\{V^{-6} q T, V^{-18} q T M^{3}, W^{-10} H^{5}, U^{-4} q T, U^{-12} q T K^{2}\right\} \\
& \leq U^{2} V^{2} W^{2}\left(V^{-6} q T\right)^{\frac{1}{3}}\left(W^{-10} H^{5}\right)^{\frac{1}{5}}\left(U^{-4} q T\right)^{\frac{9}{20}}\left(U^{-12} q T K^{2}\right)^{\frac{1}{60}} \\
& =(q T)^{\frac{4}{5}} H K^{\frac{1}{30}} \ll Y^{1-\varepsilon} .
\end{aligned}
$$

(g) $F>2 V^{-6} M^{3}, 2 W^{-10} H^{5}, F \leq 2 U^{-4} K^{2}$. Then $U^{2} V^{2} W^{2} F$

$$
\begin{aligned}
& \ll U^{2} V^{2} W^{2} \min \left\{V^{-6} q T, V^{-18} q T M^{3}, W^{-10} q T, W^{-30} q T H^{5}, U^{-4} K^{2}\right\} \\
& \leq U^{2} V^{2} W^{2}\left(V^{-6} q T\right)^{\frac{1}{3}}\left(W^{-10} q T\right)^{\frac{3}{20}}\left(W^{-30} q T H^{5}\right)^{\frac{1}{60}}\left(U^{-4} K^{2}\right)^{\frac{1}{2}} \\
& =(q T)^{\frac{1}{2}} H^{\frac{1}{12}} K \ll Y^{1-\varepsilon} .
\end{aligned}
$$

(h) $F>2 V^{-6} M^{3}, 2 W^{-10} H^{5}, 2 U^{-4} K^{2}$. Then

$$
\begin{aligned}
U^{2} V^{2} W^{2} F & \\
& \ll U^{2} V^{2} W^{2} \min \left\{V^{-6}, V^{-18} M^{3}, W^{-10}, W^{-30} H^{5}, U^{-4}, U^{-12} K^{2}\right\} q T \\
& \leq U^{2} V^{2} W^{2}\left(V^{-6}\right)^{\frac{17}{60}}\left(V^{-18} M^{3}\right)^{\frac{1}{60}}\left(W^{-10}\right)^{\frac{1}{5}}\left(U^{-4}\right)^{\frac{1}{2}} q T \\
& =q T M^{\frac{1}{20}} \ll Y^{1-\varepsilon},
\end{aligned}
$$

since $M \ll Y$. 
II. Next, we assume condition 2). On applying the mean value estimate and Halász method to $M^{2}(s, \chi) H(s, \chi), H^{5}(s, \chi)$ and $K^{2}(s, \chi)$, we get

$$
I \ll U^{2} V^{2} W^{2} Y^{-1} F \log ^{c} N,
$$

where

$$
\begin{aligned}
F=\min \{ & V^{-4} W^{-2}\left(M^{2} H+q T\right), V^{-4} W^{-2} M^{2} H+V^{-12} W^{-6} q T M^{2} H, \\
& W^{-10}\left(H^{5}+q T\right), W^{-10} H^{5}+W^{-30} q T H^{5}, U^{-4}\left(K^{2}+q T\right), \\
& \left.U^{-4} K^{2}+U^{-12} q T K^{2}\right\} .
\end{aligned}
$$

We consider several cases:

(a) $F \leq 2 V^{-4} W^{-2} M^{2} H, 2 W^{-10} H^{5}, 2 U^{-4} K^{2}$. Then

$$
\begin{aligned}
U^{2} V^{2} W^{2} F & \ll U^{2} V^{2} W^{2} \min \left\{V^{-4} W^{-2} M^{2} H, W^{-10} H^{5}, U^{-4} K^{2}\right\} \\
& \leq U^{2} V^{2} W^{2}\left(V^{-4} W^{-2} M^{2} H\right)^{\frac{1}{2}}\left(W^{-10} H^{5}\right)^{\frac{1}{12}}\left(U^{-4} K^{2}\right)^{\frac{5}{12}} \\
& =W^{\frac{1}{6}} H^{\frac{11}{12}} U^{\frac{1}{3}} K^{\frac{5}{6}} M \ll Y \log ^{-7 E} N .
\end{aligned}
$$

(b) $F \leq 2 V^{-4} W^{-2} M^{2} H, 2 W^{-10} H^{5}, F>2 U^{-4} K^{2}$. Then

$$
\begin{aligned}
& U^{2} V^{2} W^{2} F \\
& \ll U^{2} V^{2} W^{2} \min \left\{V^{-4} W^{-2} M^{2} H, W^{-10} H^{5}, U^{-4} q T, U^{-12} q T K^{2}\right\} \\
& \leq U^{2} V^{2} W^{2}\left(V^{-4} W^{-2} M^{2} H\right)^{\frac{1}{2}}\left(W^{-10} H^{5}\right)^{\frac{1}{10}}\left(U^{-4} q T\right)^{\frac{7}{20}}\left(U^{-12} q T K^{2}\right)^{\frac{1}{20}} \\
& =(q T)^{\frac{2}{5}} M H K^{\frac{1}{10}} \ll Y^{1-\varepsilon} \text {. } \\
& \text { (c) } F \leq 2 V^{-4} W^{-2} M^{2} H, F>2 W^{-10} H^{5}, F \leq 2 U^{-4} K^{2} \text {. Then } \\
& U^{2} V^{2} W^{2} F \\
& \ll U^{2} V^{2} W^{2} \min \left\{V^{-4} W^{-2} M^{2} H, W^{-10} q T, W^{-30} q T H^{5}, U^{-4} K^{2}\right\} \\
& \leq U^{2} V^{2} W^{2}\left(V^{-4} W^{-2} M^{2} H\right)^{\frac{1}{2}}\left(W^{-30} q T H^{5}\right)^{\frac{1}{30}}\left(U^{-4} K^{2}\right)^{\frac{7}{15}} \\
& =(q T)^{\frac{1}{30}} U^{\frac{2}{15}} K^{\frac{14}{15}} M H^{\frac{2}{3}} \\
& \ll(q T)^{\frac{1}{30}} M H^{\frac{2}{3}} K \ll Y^{1-\varepsilon} . \\
& \text { (d) } F \leq 2 V^{-4} W^{-2} M^{2} H, F>2 W^{-10} H^{5}, 2 U^{-4} K^{2} \text {. Then } \\
& U^{2} V^{2} W^{2} F \\
& \ll U^{2} V^{2} W^{2} \min \left\{V^{-4} W^{-2} M^{2} H, W^{-10} q T, W^{-30} q T H^{5},\right. \\
& \left.U^{-4} q T, U^{-12} q T K^{2}\right\} \\
& \leq U^{2} V^{2} W^{2}\left(V^{-4} W^{-2} M^{2} H\right)^{\frac{1}{2}}\left(W^{-30} q T H^{5}\right)^{\frac{1}{30}}\left(U^{-4} q T\right)^{\frac{9}{20}}\left(U^{-12} q T K^{2}\right)^{\frac{1}{60}} \\
& =(q T)^{\frac{1}{2}} M H^{\frac{2}{3}} K^{\frac{1}{30}} \ll Y^{1-\varepsilon} \text {. }
\end{aligned}
$$


(e) $F>2 V^{-4} W^{-2} M^{2} H, F \leq 2 W^{-10} H^{5}, 2 U^{-4} K^{2}$. Then

$$
\begin{aligned}
& U^{2} V^{2} W^{2} F \\
& \ll U^{2} V^{2} W^{2} \min \left\{V^{-4} W^{-2} q T, V^{-12} W^{-6} q T M^{2} H, W^{-10} H^{5}, U^{-4} K^{2}\right\} \\
& \leq U^{2} V^{2} W^{2}\left(V^{-4} W^{-2} q T\right)^{\frac{7}{20}}\left(V^{-12} W^{-6} q T M^{2} H\right)^{\frac{1}{20}}\left(W^{-10} H^{5}\right)^{\frac{1}{10}}\left(U^{-4} K^{2}\right)^{\frac{1}{2}} \\
& =(q T)^{\frac{2}{5}} M^{\frac{1}{10}} H^{\frac{11}{20}} K \ll Y^{1-\varepsilon} \text {. } \\
& \text { (f) } F>2 V^{-4} W^{-2} M^{2} H, F \leq 2 W^{-10} H^{5}, F>2 U^{-4} K^{2} \text {. Then } \\
& U^{2} V^{2} W^{2} F \\
& \ll U^{2} V^{2} W^{2} \min \left\{V^{-4} W^{-2} q T, V^{-12} W^{-6} q T M^{2} H, W^{-10} H^{5}, U^{-4} q T,\right. \\
& \left.U^{-12} q T K^{2}\right\} \\
& \leq U^{2} V^{2} W^{2}\left(V^{-4} W^{-2} q T\right)^{\frac{1}{2}}\left(W^{-10} H^{5}\right)^{\frac{1}{10}}\left(U^{-4} q T\right)^{\frac{7}{20}}\left(U^{-12} q T K^{2}\right)^{\frac{1}{20}} \\
& =(q T)^{\frac{9}{10}} H^{\frac{1}{2}} K^{\frac{1}{10}} \ll Y^{1-\varepsilon} \text {. } \\
& \ll U^{2} V^{2} W^{2} \min \left\{V^{-4} W^{-2} q T, V^{-12} W^{-6} q T M^{2} H, W^{-10} q T, W^{-30} q T H^{5},\right. \\
& \left.U^{-4} K^{2}\right\} \\
& \leq U^{2} V^{2} W^{2}\left(V^{-4} W^{-2} q T\right)^{\frac{9}{20}}\left(V^{-12} W^{-6} q T M^{2} H\right)^{\frac{1}{60}}\left(W^{-30} q T H^{5}\right)^{\frac{1}{30}} \\
& \times\left(U^{-4} K^{2}\right)^{\frac{1}{2}} \\
& =(q T)^{\frac{1}{2}} M^{\frac{1}{30}} H^{\frac{11}{60}} K \ll Y^{1-\varepsilon} \text {. } \\
& \text { (h) } F>2 V^{-4} W^{-2} M^{2} H, 2 W^{-10} H^{5}, 2 U^{-4} K^{2} \text {. Then } \\
& U^{2} V^{2} W^{2} F \ll U^{2} V^{2} W^{2} \min \left\{V^{-4} W^{-2}, V^{-12} W^{-6} M^{2} H, W^{-10}, W^{-30} H^{5}\right. \text {, } \\
& \left.U^{-4}, U^{-12} K^{2}\right\} q T \\
& \leq U^{2} V^{2} W^{2}\left(V^{-4} W^{-2}\right)^{\frac{1}{2}}\left(W^{-10}\right)^{\frac{1}{10}}\left(U^{-4}\right)^{\frac{7}{20}}\left(U^{-12} K^{2}\right)^{\frac{1}{20}} q T \\
& =q T K^{\frac{1}{10}} \ll Y^{1-\varepsilon} \text {, }
\end{aligned}
$$

since $Y^{\frac{4}{9}} \ll M H$ (the latter follows from $Y^{\frac{16}{135}} \ll H$ and $Y^{\frac{68}{81}} \ll M^{2} H$ ).

III. Now, we assume condition 3$)$. On applying the mean value estimate and Halász method to $M^{3}(s, \chi), H^{4}(s, \chi)$ and $K^{2}(s, \chi)$, we get

$$
I \ll U^{2} V^{2} W^{2} Y^{-1} F \log ^{c} N,
$$

where

$$
\begin{gathered}
F=\min \left\{V^{-6}\left(M^{3}+q T\right), V^{-6} M^{3}+V^{-18} q T M^{3}, W^{-8}\left(H^{4}+q T\right), W^{-8} H^{4}\right. \\
\left.+W^{-24} q T H^{4}, U^{-4}\left(K^{2}+q T\right), U^{-4} K^{2}+U^{-12} q T K^{2}\right\} .
\end{gathered}
$$


Consider the following cases:

(a) $F \leq 2 V^{-6} M^{3}, 2 W^{-8} H^{4}, 2 U^{-4} K^{2}$. Then

$$
\begin{aligned}
U^{2} V^{2} W^{2} F & \ll U^{2} V^{2} W^{2} \min \left\{V^{-6} M^{3}, W^{-8} H^{4}, U^{-4} K^{2}\right\} \\
& \leq U^{2} V^{2} W^{2}\left(V^{-6} M^{3}\right)^{\frac{3}{10}}\left(W^{-8} H^{4}\right)^{\frac{1}{4}}\left(U^{-4} K^{2}\right)^{\frac{9}{20}} \\
& =V^{\frac{1}{5}} M^{\frac{9}{10}} U^{\frac{1}{5}} K^{\frac{9}{10}} H \ll Y \log ^{-7 E} N .
\end{aligned}
$$

(b) $F \leq 2 V^{-6} M^{3}, 2 W^{-8} H^{4}, F>2 U^{-4} K^{2}$. Then

$$
\begin{aligned}
U^{2} V^{2} W^{2} F & \ll U^{2} V^{2} W^{2} \min \left\{V^{-6} M^{3}, W^{-8} H^{4}, U^{-4} q T, U^{-12} q T K^{2}\right\} \\
& \leq U^{2} V^{2} W^{2}\left(V^{-6} M^{3}\right)^{\frac{1}{3}}\left(W^{-8} H^{4}\right)^{\frac{1}{4}}\left(U^{-4} q T\right)^{\frac{3}{8}}\left(U^{-12} q T K^{2}\right)^{\frac{1}{24}} \\
& =(q T)^{\frac{5}{12}} M H K^{\frac{1}{12}} \ll Y^{1-\varepsilon} .
\end{aligned}
$$

(c) $F \leq 2 V^{-6} M^{3}, F>2 W^{-8} H^{4}, F \leq 2 U^{-4} K^{2}$. Then

$$
\begin{aligned}
U^{2} V^{2} W^{2} F & \ll U^{2} V^{2} W^{2} \min \left\{V^{-6} M^{3}, W^{-8} q T, W^{-24} q T H^{4}, U^{-4} K^{2}\right\} \\
& \leq U^{2} V^{2} W^{2}\left(V^{-6} M^{3}\right)^{\frac{1}{3}}\left(W^{-8} q T\right)^{\frac{1}{8}}\left(W^{-24} q T H^{4}\right)^{\frac{1}{24}}\left(U^{-4} K^{2}\right)^{\frac{1}{2}} \\
& =(q T)^{\frac{1}{6}} M K H^{\frac{1}{6}} \ll Y^{1-\varepsilon} .
\end{aligned}
$$

(d) $F \leq 2 V^{-6} M^{3}, F>2 W^{-8} H^{4}, 2 U^{-4} K^{2}$. Then

$$
\begin{aligned}
U^{2} V^{2} & W^{2} F \\
& \ll U^{2} V^{2} W^{2} \min \left\{V^{-6} M^{3}, W^{-8} q T, W^{-24} q T H^{4}, U^{-4} q T, U^{-12} q T K^{2}\right\} \\
& \leq U^{2} V^{2} W^{2}\left(V^{-6} M^{3}\right)^{\frac{1}{3}}\left(W^{-8} q T\right)^{\frac{1}{8}}\left(W^{-24} q T H^{4}\right)^{\frac{1}{24}}\left(U^{-4} q T\right)^{\frac{1}{2}} \\
& =(q T)^{\frac{2}{3}} M H^{\frac{1}{6}} \ll Y^{1-\varepsilon} .
\end{aligned}
$$

(e) $F>2 V^{-6} M^{3}, F \leq 2 W^{-8} H^{4}, 2 U^{-4} K^{2}$. Then

$$
\begin{aligned}
U^{2} V^{2} W^{2} F & \ll U^{2} V^{2} W^{2} \min \left\{V^{-6} q T, V^{-18} q T M^{3}, W^{-8} H^{4}, U^{-4} K^{2}\right\} \\
& \leq U^{2} V^{2} W^{2}\left(V^{-6} q T\right)^{\frac{5}{24}}\left(V^{-18} q T M^{3}\right)^{\frac{1}{24}}\left(W^{-8} H^{4}\right)^{\frac{1}{4}}\left(U^{-4} K^{2}\right)^{\frac{1}{2}} \\
& =(q T)^{\frac{1}{4}} M^{\frac{1}{8}} H K \ll Y^{1-\varepsilon} .
\end{aligned}
$$

(f) $F>2 V^{-6} M^{3}, F \leq 2 W^{-8} H^{4}, F>2 U^{-4} K^{2}$. Then $U^{2} V^{2} W^{2} F$

$$
\begin{aligned}
& \ll U^{2} V^{2} W^{2} \min \left\{V^{-6} q T, V^{-18} q T M^{3}, W^{-8} H^{4}, U^{-4} q T, U^{-12} q T K^{2}\right\} \\
& \leq U^{2} V^{2} W^{2}\left(V^{-6} q T\right)^{\frac{5}{24}}\left(V^{-18} q T M^{3}\right)^{\frac{1}{24}}\left(W^{-8} H^{4}\right)^{\frac{1}{4}}\left(U^{-4} q T\right)^{\frac{1}{2}} \\
& =(q T)^{\frac{3}{4}} M^{\frac{1}{8}} H \ll Y^{1-\varepsilon} .
\end{aligned}
$$


(g) $F>2 V^{-6} M^{3}, 2 W^{-8} H^{4}, F \leq 2 U^{-4} K^{2}$. Then

$U^{2} V^{2} W^{2} F$

$$
\begin{aligned}
& \ll U^{2} V^{2} W^{2} \min \left\{V^{-6} q T, V^{-18} q T M^{3}, W^{-8} q T, W^{-24} q T H^{4}, U^{-4} K^{2}\right\} \\
& \leq U^{2} V^{2} W^{2}\left(V^{-6} q T\right)^{\frac{1}{3}}\left(W^{-8} q T\right)^{\frac{1}{8}}\left(W^{-24} q T H^{4}\right)^{\frac{1}{24}}\left(U^{-4} K^{2}\right)^{\frac{1}{2}} \\
& =(q T)^{\frac{1}{2}} H^{\frac{1}{6}} K \ll Y^{1-\varepsilon} .
\end{aligned}
$$

(h) $F>2 V^{-6} M^{3}, 2 W^{-8} H^{4}, 2 U^{-4} K^{2}$. Then

$U^{2} V^{2} W^{2} F$

$$
\begin{aligned}
& \ll U^{2} V^{2} W^{2} \min \left\{V^{-6}, V^{-18} M^{3}, W^{-8}, W^{-24} H^{4}, U^{-4}, U^{-12} K^{2}\right\} q T \\
& \leq U^{2} V^{2} W^{2}\left(V^{-6}\right)^{\frac{5}{24}}\left(V^{-18} M^{3}\right)^{\frac{1}{24}}\left(W^{-8}\right)^{\frac{1}{4}}\left(U^{-4}\right)^{\frac{1}{2}} q T \\
& =q T M^{\frac{1}{8}} \ll Y^{1-\varepsilon},
\end{aligned}
$$

since $M \ll Y^{\frac{4}{9}}$ (the latter follows from $M H \ll Y^{\frac{113}{198}}$ and $Y^{\frac{17}{90}} \ll H$ ).

IV. Lastly, condition 4$)$ is assumed. On applying the mean value estimate and Halász method to $M^{2}(s, \chi) H(s, \chi), H^{4}(s, \chi)$ and $K^{2}(s, \chi)$, we get

$$
I \ll U^{2} V^{2} W^{2} Y^{-1} F \log ^{c} N,
$$

where

$$
\begin{aligned}
F=\min \{ & V^{-4} W^{-2}\left(M^{2} H+q T\right), V^{-4} W^{-2} M^{2} H+V^{-12} W^{-6} q T M^{2} H, \\
& W^{-8}\left(H^{4}+q T\right), W^{-8} H^{4}+W^{-24} q T H^{4}, U^{-4}\left(K^{2}+q T\right), \\
& \left.U^{-4} K^{2}+U^{-12} q T K^{2}\right\} .
\end{aligned}
$$

We consider the following cases:

(a) $F \leq 2 V^{-4} W^{-2} M^{2} H, 2 W^{-8} H^{4}, 2 U^{-4} K^{2}$. Then

$$
\begin{aligned}
U^{2} V^{2} W^{2} F & \ll U^{2} V^{2} W^{2} \min \left\{V^{-4} W^{-2} M^{2} H, W^{-8} H^{4}, U^{-4} K^{2}\right\} \\
& \leq U^{2} V^{2} W^{2}\left(V^{-4} W^{-2} M^{2} H\right)^{\frac{1}{2}}\left(W^{-8} H^{4}\right)^{\frac{1}{12}}\left(U^{-4} K^{2}\right)^{\frac{5}{12}} \\
& =W^{\frac{1}{3}} H^{\frac{5}{6}} U^{\frac{1}{3}} K^{\frac{5}{6}} M \ll Y \log ^{-7 E} N .
\end{aligned}
$$

(b) $F \leq 2 V^{-4} W^{-2} M^{2} H, 2 W^{-8} H^{4}, F>2 U^{-4} K^{2}$. Then

$$
\begin{aligned}
U^{2} V^{2} W^{2} F & \\
& \ll U^{2} V^{2} W^{2} \min \left\{V^{-4} W^{-2} M^{2} H, W^{-8} H^{4}, U^{-4} q T, U^{-12} q T K^{2}\right\} \\
& \leq U^{2} V^{2} W^{2}\left(V^{-4} W^{-2} M^{2} H\right)^{\frac{1}{2}}\left(W^{-8} H^{4}\right)^{\frac{1}{8}}\left(U^{-4} q T\right)^{\frac{5}{16}}\left(U^{-12} q T K^{2}\right)^{\frac{1}{16}} \\
& =(q T)^{\frac{3}{8}} M H K^{\frac{1}{8}} \ll Y^{1-\varepsilon} .
\end{aligned}
$$


(c) $F \leq 2 V^{-4} W^{-2} M^{2} H, F>2 W^{-8} H^{4}, F \leq 2 U^{-4} K^{2}$. Then $U^{2} V^{2} W^{2} F$

$$
\begin{aligned}
& \ll U^{2} V^{2} W^{2} \min \left\{V^{-4} W^{-2} M^{2} H, W^{-8} q T, W^{-24} q T H^{4}, U^{-4} K^{2}\right\} \\
& \leq U^{2} V^{2} W^{2}\left(V^{-4} W^{-2} M^{2} H\right)^{\frac{1}{2}}\left(W^{-24} q T H^{4}\right)^{\frac{1}{24}}\left(U^{-4} K^{2}\right)^{\frac{11}{24}} \\
& =(q T)^{\frac{1}{24}} U^{\frac{1}{6}} K^{\frac{11}{12}} M H^{\frac{2}{3}} \\
& \ll(q T)^{\frac{1}{24}} M H^{\frac{2}{3}} K \ll Y^{1-\varepsilon} . \\
\text { (d) } F \leq & 2 V^{-4} W^{-2} M^{2} H, F>2 W^{-8} H^{4}, 2 U^{-4} K^{2} . \text { Then }
\end{aligned}
$$

$$
\begin{aligned}
& U^{2} V^{2} W^{2} F \\
& \quad \ll U^{2} V^{2} W^{2} \min \left\{V^{-4} W^{-2} M^{2} H, W^{-8} q T, W^{-24} q T H^{4}, U^{-4} q T,\right.
\end{aligned}
$$

$$
\left.U^{-12} q T K^{2}\right\}
$$

$\leq U^{2} V^{2} W^{2}\left(V^{-4} W^{-2} M^{2} H\right)^{\frac{1}{2}}\left(W^{-24} q T H^{4}\right)^{\frac{1}{24}}\left(U^{-4} q T\right)^{\frac{7}{16}}\left(U^{-12} q T K^{2}\right)^{\frac{1}{48}}$

$=(q T)^{\frac{1}{2}} M H^{\frac{2}{3}} K^{\frac{1}{24}} \ll Y^{1-\varepsilon}$.

(e) $F>2 V^{-4} W^{-2} M^{2} H, F \leq 2 W^{-8} H^{4}, 2 U^{-4} K^{2}$. Then

$U^{2} V^{2} W^{2} F$

$\ll U^{2} V^{2} W^{2} \min \left\{V^{-4} W^{-2} q T, V^{-12} W^{-6} q T M^{2} H, W^{-8} H^{4}, U^{-4} K^{2}\right\}$

$\leq U^{2} V^{2} W^{2}\left(V^{-4} W^{-2} q T\right)^{\frac{5}{16}}\left(V^{-12} W^{-6} q T M^{2} H\right)^{\frac{1}{16}}\left(W^{-8} H^{4}\right)^{\frac{1}{8}}\left(U^{-4} K^{2}\right)^{\frac{1}{2}}$

$=(q T)^{\frac{3}{8}} M^{\frac{1}{8}} H^{\frac{9}{16}} K \ll Y^{1-\varepsilon}$.

(f) $F>2 V^{-4} W^{-2} M^{2} H, F \leq 2 W^{-8} H^{4}, F>2 U^{-4} K^{2}$. Then

$U^{2} V^{2} W^{2} F$

$$
\begin{aligned}
& \ll U^{2} V^{2} W^{2} \min \left\{V^{-4} W^{-2} q T, V^{-12} W^{-6} q T M^{2} H, W^{-8} H^{4}, U^{-4} q T,\right. \\
& \left.\qquad U^{-12} q T K^{2}\right\} \\
& \leq U^{2} V^{2} W^{2}\left(V^{-4} W^{-2} q T\right)^{\frac{1}{2}}\left(W^{-8} H^{4}\right)^{\frac{1}{8}}\left(U^{-4} q T\right)^{\frac{5}{16}}\left(U^{-12} q T K^{2}\right)^{\frac{1}{16}} \\
& =(q T)^{\frac{7}{8}} H^{\frac{1}{2}} K^{\frac{1}{8}} \ll Y^{1-\varepsilon} .
\end{aligned}
$$

(g) $F>2 V^{-4} W^{-2} M^{2} H, 2 W^{-8} H^{4}, F \leq 2 U^{-4} K^{2}$. Then $U^{2} V^{2} W^{2} F$

$\ll U^{2} V^{2} W^{2} \min \left\{V^{-4} W^{-2} q T, V^{-12} W^{-6} q T M^{2} H, W^{-8} q T\right.$,

$$
\left.W^{-24} q T H^{4}, U^{-4} K^{2}\right\}
$$

$\leq U^{2} V^{2} W^{2}\left(V^{-4} W^{-2} q T\right)^{\frac{5}{16}}\left(V^{-12} W^{-6} q T M^{2} H\right)^{\frac{1}{16}}\left(W^{-8} q T\right)^{\frac{1}{8}}\left(U^{-4} K^{2}\right)^{\frac{1}{2}}$

$=(q T)^{\frac{1}{2}} M^{\frac{1}{8}} H^{\frac{1}{16}} K \ll Y^{1-\varepsilon}$. 
(h) $F>2 V^{-4} W^{-2} M^{2} H, 2 W^{-8} H^{4}, 2 U^{-4} K^{2}$. Then

$$
\begin{aligned}
U^{2} V^{2} W^{2} F & \ll U^{2} V^{2} W^{2} \min \left\{V^{-4} W^{-2}, V^{-12} W^{-6} M^{2} H, W^{-8}, W^{-24} H^{4},\right. \\
& \left.U^{-4}, U^{-12} K^{2}\right\} q T \\
\leq & U^{2} V^{2} W^{2}\left(V^{-4} W^{-2}\right)^{\frac{1}{2}}\left(W^{-8}\right)^{\frac{1}{8}}\left(U^{-4}\right)^{\frac{5}{16}}\left(U^{-12} K^{2}\right)^{\frac{1}{16}} q T \\
& =q T K^{\frac{1}{8}} \ll Y^{1-\varepsilon} .
\end{aligned}
$$

Combining the above, we obtain (9). Hence, Lemma 7 follows.

Lemma 8. Under the assumption of Lemma $7, M$ and $H$ lie in one of the following regions:

(i) $\quad Y^{\frac{17}{63}} \ll M \ll Y^{\frac{17}{57}}, \quad M^{-\frac{6}{5}} Y^{\frac{17}{30}} \ll H \ll M^{-\frac{1}{8}} Y^{\frac{7}{24}}$;

(ii) $\quad Y^{\frac{17}{57}} \ll M \ll Y^{\frac{17}{54}}, \quad M^{-\frac{12}{11}} Y^{\frac{17}{33}} \ll H \ll M^{-\frac{1}{8}} Y^{\frac{7}{24}}$;

(iii) $\quad Y^{\frac{17}{54}} \ll M \ll Y^{\frac{221}{693}}, \quad Y^{\frac{17}{99}} \ll H \ll M^{-\frac{1}{8}} Y^{\frac{7}{24}} ;$

(iv) $\quad Y^{\frac{221}{693}} \ll M \ll Y^{\frac{109}{330}}, \quad Y^{\frac{17}{99}} \ll H \ll M^{-1} Y^{\frac{113}{198}}$;

(v) $\quad Y^{\frac{109}{330}} \ll M \ll Y^{\frac{151}{450}}, \quad Y^{\frac{17}{99}} \ll H \ll M^{-6} Y^{\frac{20}{9}} ;$

(vi) $\quad Y^{\frac{151}{450}} \ll M \ll Y^{\frac{34}{99}}, \quad Y^{\frac{17}{99}} \ll H \ll M^{\frac{1}{4}} Y^{\frac{1}{8}}$;

(vii) $\quad Y^{\frac{151}{450}} \ll M \ll Y^{\frac{34}{99}}, \quad M^{-1} Y^{\frac{5}{9}} \ll H \ll M^{\frac{1}{3}} Y^{\frac{7}{54}}$;

(viii) $\quad Y^{\frac{34}{99}} \ll M \ll Y^{\frac{25}{72}}, \quad M^{-\frac{58}{49}} Y^{\frac{85}{147}} \ll H \ll M^{\frac{1}{4}} Y^{\frac{1}{8}}$;

(ix) $\quad Y^{\frac{25}{72}} \ll M \ll Y^{\frac{10}{27}}, \quad M^{-\frac{58}{49}} Y^{\frac{85}{147}} \ll H \ll M^{-\frac{23}{15}} Y^{\frac{7}{9}} ;$

(x) $\quad Y^{\frac{10}{27}} \ll M \ll Y^{\frac{617}{1620}}, \quad M^{-\frac{58}{49}} Y^{\frac{85}{147}} \ll H \ll M^{-1} Y^{\frac{47}{81}} ;$

(xi) $\quad Y^{\frac{617}{1620}} \ll M \ll Y^{\frac{3041}{7830}}, \quad M^{-\frac{58}{49}} Y^{\frac{85}{147}} \ll H \ll M^{-\frac{29}{19}} Y^{\frac{89}{114}}$;

(xii) $\quad Y^{\frac{3041}{7830}} \ll M \ll Y^{\frac{3397}{7830}}, \quad Y^{\frac{16}{135}} \ll H \ll M^{-\frac{29}{19}} Y^{\frac{89}{114}}$.

Then (8) holds for $T_{0} \leq T \leq Y$.

Proof. In the regions:

$$
\begin{array}{ll}
Y^{\frac{17}{57}} \ll M \ll Y^{\frac{17}{54}}, & M^{-\frac{12}{11}} Y^{\frac{17}{33}} \ll H \ll M^{\frac{1}{29}} Y^{\frac{19}{87}} \\
Y^{\frac{17}{54}} \ll M \ll Y^{\frac{34}{99}}, & Y^{\frac{17}{99}} \ll H \ll M^{-1} Y^{\frac{142}{261}},
\end{array}
$$

we apply Lemma 7 with condition 1).

In the regions:

$$
\begin{array}{ll}
Y^{\frac{175}{522}} \ll M \ll Y^{\frac{34}{99}}, & M^{-1} Y^{\frac{142}{261}} \ll H \ll M^{\frac{1}{4}} Y^{\frac{1}{8}} ; \\
Y^{\frac{34}{99}} \ll M \ll Y^{\frac{59}{162}}, & M^{-\frac{58}{49}} Y^{\frac{85}{147}} \ll H \ll M^{\frac{1}{4}} Y^{\frac{1}{8}} ; \\
Y^{\frac{59}{162}} \ll M \ll Y^{\frac{617}{1620}}, & M^{-\frac{58}{49}} Y^{\frac{85}{147}} \ll H \ll M^{-1} Y^{\frac{47}{81}} ;
\end{array}
$$




$$
\begin{array}{ll}
Y^{\frac{617}{1620}} \ll M \ll Y^{\frac{3041}{7830}}, & M^{-\frac{58}{49}} Y^{\frac{85}{147}} \ll H \ll M^{-\frac{29}{19}} Y^{\frac{89}{114}} \\
Y^{\frac{3041}{7830}} \ll M \ll Y^{\frac{3397}{7830}}, & Y^{\frac{16}{135}} \ll H \ll M^{-\frac{29}{19}} Y^{\frac{89}{114}},
\end{array}
$$

we apply Lemma 7 with condition 2).

In the regions:

$$
\begin{array}{ll}
Y^{\frac{17}{63}} \ll M \ll Y^{\frac{17}{57}}, & M^{-\frac{6}{5}} Y^{\frac{17}{30}} \ll H \ll M^{-\frac{1}{8}} Y^{\frac{7}{24}} ; \\
Y^{\frac{17}{57}} \ll M \ll Y^{\frac{17}{54}}, & M^{\frac{1}{29}} Y^{\frac{19}{87}} \ll H \ll M^{-\frac{1}{8}} Y^{\frac{7}{24}} ; \\
Y^{\frac{17}{54}} \ll M \ll Y^{\frac{221}{693}}, & M^{-1} Y^{\frac{142}{261}} \ll H \ll M^{-\frac{1}{8}} Y^{\frac{7}{24}} ; \\
Y^{\frac{221}{693}} \ll M \ll Y^{\frac{109}{330}}, & M^{-1} Y^{\frac{142}{261}} \ll H \ll M^{-1} Y^{\frac{113}{198}} ; \\
Y^{\frac{109}{330}} \ll M \ll Y^{\frac{175}{522}}, & M^{-1} Y^{\frac{142}{261}} \ll H \ll M^{-6} Y^{\frac{20}{9}} ; \\
Y^{\frac{175}{522}} \ll M \ll Y^{\frac{151}{450}}, & M^{\frac{1}{4}} Y^{\frac{1}{8}} \ll H \ll M^{-6} Y^{\frac{20}{9}},
\end{array}
$$

we apply Lemma 7 with condition 3).

In the regions:

$$
\begin{array}{ll}
Y^{\frac{151}{450}} \ll M \ll Y^{\frac{34}{99}}, & M^{-1} Y^{\frac{5}{9}} \ll H \ll M^{\frac{1}{3}} Y^{\frac{7}{54}} \\
Y^{\frac{25}{72}} \ll M \ll Y^{\frac{59}{162}}, & M^{\frac{1}{4}} Y^{\frac{1}{8}} \ll H \ll M^{-\frac{23}{15}} Y^{\frac{7}{9}} \\
Y^{\frac{59}{162}} \ll M \ll Y^{\frac{10}{27}}, & M^{-1} Y^{\frac{47}{81}} \ll H \ll M^{-\frac{23}{15}} Y^{\frac{7}{9}},
\end{array}
$$

we apply Lemma 7 with condition 4).

Putting together the above regions, we get Lemma 8.

Lemma 9. Under the assumption of Lemma 7, suppose that $M$ and $H$ also satisfy one of the following four conditions:

1) $M H \ll Y^{\frac{40}{57}}, Y^{\frac{17}{99}} \ll H, M \ll Y^{\frac{1}{2}}, Y^{\frac{119}{261}} \ll M, H^{19} / M \ll Y^{\frac{35}{9}}$, $Y^{\frac{68}{99}} \ll M^{\frac{12}{11}} H$

2) $M^{2} H \ll Y^{\frac{94}{81}}, M^{\frac{58}{9}} H \ll Y^{\frac{89}{27}}, Y^{\frac{34}{81}} \ll M, M^{\frac{1}{5}} H \ll Y^{\frac{3}{10}}, Y^{\frac{17}{12}} \ll$ $M^{\frac{29}{10}} H, Y^{\frac{16}{135}} \ll H$

3) $M H \ll Y^{\frac{46}{63}}, Y^{\frac{17}{90}} \ll H, M^{6} H \ll Y^{\frac{19}{6}}, Y^{\frac{85}{198}} \ll M, H^{7} / M \ll Y^{\frac{4}{3}}$, $Y^{\frac{34}{45}} \ll M^{\frac{6}{5}} H$

4) $M^{2} H \ll Y^{\frac{25}{21}}, M^{\frac{46}{7}} H \ll Y^{\frac{10}{3}}, Y^{\frac{17}{42}} \ll M, M^{\frac{1}{4}} H \ll Y^{\frac{25}{72}}, Y^{\frac{17}{12}} \ll$ $M^{\frac{23}{8}} H, M \ll Y^{\frac{4}{9}}$.

Then (8) holds for $T_{0} \leq T \leq Y$.

Proof. We only show that (9) holds for $T=1 / \eta=Y /(q Q)$.

I. First, we assume condition 1$)$. We apply the mean value estimate and Halász method to $M^{2}(s, \chi), H^{5}(s, \chi)$ and $K^{3}(s, \chi)$ to get

$$
I \ll U^{2} V^{2} W^{2} Y^{-1} F \log ^{c} N,
$$


where

$$
\begin{aligned}
F=\min \left\{V^{-4}\left(M^{2}+q T\right), V^{-4} M^{2}+V^{-12} q T M^{2}, W^{-10}\left(H^{5}+q T\right),\right. \\
\left.W^{-10} H^{5}+W^{-30} q T H^{5}, U^{-6}\left(K^{3}+q T\right), U^{-6} K^{3}+U^{-18} q T K^{3}\right\} .
\end{aligned}
$$

We consider several cases:

(a) $F \leq 2 V^{-4} M^{2}, 2 W^{-10} H^{5}, 2 U^{-6} K^{3}$. Then

$$
\begin{aligned}
U^{2} V^{2} W^{2} F & \ll U^{2} V^{2} W^{2} \min \left\{V^{-4} M^{2}, W^{-10} H^{5}, U^{-6} K^{3}\right\} \\
& \leq U^{2} V^{2} W^{2}\left(V^{-4} M^{2}\right)^{\frac{1}{2}}\left(W^{-10} H^{5}\right)^{\frac{1}{6}}\left(U^{-6} K^{3}\right)^{\frac{1}{3}} \\
& =W^{\frac{1}{3}} H^{\frac{5}{6}} M K \ll Y \log ^{-7 E} N .
\end{aligned}
$$

(b) $F \leq 2 V^{-4} M^{2}, 2 W^{-10} H^{5}, F>2 U^{-6} K^{3}$. Then

$$
\begin{aligned}
U^{2} V^{2} W^{2} F & \ll U^{2} V^{2} W^{2} \min \left\{V^{-4} M^{2}, W^{-10} H^{5}, U^{-6} q T, U^{-18} q T K^{3}\right\} \\
& \leq U^{2} V^{2} W^{2}\left(V^{-4} M^{2}\right)^{\frac{1}{2}}\left(W^{-10} H^{5}\right)^{\frac{1}{5}}\left(U^{-6} q T\right)^{\frac{17}{60}}\left(U^{-18} q T K^{3}\right)^{\frac{1}{60}} \\
& =(q T)^{\frac{3}{10}} M H K^{\frac{1}{20}} \ll Y^{1-\varepsilon} .
\end{aligned}
$$

(c) $F \leq 2 V^{-4} M^{2}, F>2 W^{-10} H^{5}, F \leq 2 U^{-6} K^{3}$. Then

$$
\begin{aligned}
U^{2} V^{2} W^{2} F & \ll U^{2} V^{2} W^{2} \min \left\{V^{-4} M^{2}, W^{-10} q T, W^{-30} q T H^{5}, U^{-6} K^{3}\right\} \\
& \leq U^{2} V^{2} W^{2}\left(V^{-4} M^{2}\right)^{\frac{1}{2}}\left(W^{-10} q T\right)^{\frac{3}{20}}\left(W^{-30} q T H^{5}\right)^{\frac{1}{60}}\left(U^{-6} K^{3}\right)^{\frac{1}{3}} \\
& =(q T)^{\frac{1}{6}} M K H^{\frac{1}{12}} \ll Y^{1-\varepsilon} .
\end{aligned}
$$

(d) $F \leq 2 V^{-4} M^{2}, F>2 W^{-10} H^{5}, 2 U^{-6} K^{3}$. Then

$U^{2} V^{2} W^{2} F$

$$
\begin{aligned}
& \ll U^{2} V^{2} W^{2} \min \left\{V^{-4} M^{2}, W^{-10} q T, W^{-30} q T H^{5}, U^{-6} q T, U^{-18} q T K^{3}\right\} \\
& \leq U^{2} V^{2} W^{2}\left(V^{-4} M^{2}\right)^{\frac{1}{2}}\left(W^{-10} q T\right)^{\frac{1}{5}}\left(U^{-6} q T\right)^{\frac{17}{60}}\left(U^{-18} q T K^{3}\right)^{\frac{1}{60}} \\
& =(q T)^{\frac{1}{2}} M K^{\frac{1}{20}} \ll Y^{1-\varepsilon},
\end{aligned}
$$

since $M^{19} / H \ll Y^{\frac{86}{9}}$ (the latter follows from $M \ll Y^{\frac{1}{2}}$ ).

(e) $F>2 V^{-4} M^{2}, F \leq 2 W^{-10} H^{5}, 2 U^{-6} K^{3}$. Then

$$
\begin{aligned}
U^{2} V^{2} W^{2} F & \ll U^{2} V^{2} W^{2} \min \left\{V^{-4} q T, V^{-12} q T M^{2}, W^{-10} H^{5}, U^{-6} K^{3}\right\} \\
& \leq U^{2} V^{2} W^{2}\left(V^{-4} q T\right)^{\frac{9}{20}}\left(V^{-12} q T M^{2}\right)^{\frac{1}{60}}\left(W^{-10} H^{5}\right)^{\frac{1}{5}}\left(U^{-6} K^{3}\right)^{\frac{1}{3}} \\
& =(q T)^{\frac{7}{15}} M^{\frac{1}{30}} H K \ll Y^{1-\varepsilon} .
\end{aligned}
$$


(f) $F>2 V^{-4} M^{2}, F \leq 2 W^{-10} H^{5}, F>2 U^{-6} K^{3}$. Then

$$
\begin{aligned}
U^{2} V^{2} & W^{2} F \\
& \ll U^{2} V^{2} W^{2} \min \left\{V^{-4} q T, V^{-12} q T M^{2}, W^{-10} H^{5}, U^{-6} q T, U^{-18} q T K^{3}\right\} \\
& \leq U^{2} V^{2} W^{2}\left(V^{-4} q T\right)^{\frac{1}{2}}\left(W^{-10} H^{5}\right)^{\frac{1}{5}}\left(U^{-6} q T\right)^{\frac{17}{60}}\left(U^{-18} q T K^{3}\right)^{\frac{1}{60}} \\
& =(q T)^{\frac{4}{5}} H K^{\frac{1}{20}} \ll Y^{1-\varepsilon} .
\end{aligned}
$$

(g) $F>2 V^{-4} M^{2}, 2 W^{-10} H^{5}, F \leq 2 U^{-6} K^{3}$. Then

$U^{2} V^{2} W^{2} F$

$$
\begin{aligned}
& \ll U^{2} V^{2} W^{2} \min \left\{V^{-4} q T, V^{-12} q T M^{2}, W^{-10} q T, W^{-30} q T H^{5}, U^{-6} K^{3}\right\} \\
& \leq U^{2} V^{2} W^{2}\left(V^{-4} q T\right)^{\frac{1}{2}}\left(W^{-10} q T\right)^{\frac{3}{20}}\left(W^{-30} q T H^{5}\right)^{\frac{1}{60}}\left(U^{-6} K^{3}\right)^{\frac{1}{3}} \\
& =(q T)^{\frac{2}{3}} H^{\frac{1}{12}} K \ll Y^{1-\varepsilon} .
\end{aligned}
$$

(h) $F>2 V^{-4} M^{2}, 2 W^{-10} H^{5}, 2 U^{-6} K^{3}$. Then

$$
\begin{aligned}
U^{2} V^{2} W^{2} F & \\
& \ll U^{2} V^{2} W^{2} \min \left\{V^{-4}, V^{-12} M^{2}, W^{-10}, W^{-30} H^{5}, U^{-6}, U^{-18} K^{3}\right\} q T \\
& \leq U^{2} V^{2} W^{2}\left(V^{-4}\right)^{\frac{1}{2}}\left(W^{-10}\right)^{\frac{1}{5}}\left(U^{-6}\right)^{\frac{17}{60}}\left(U^{-18} K^{3}\right)^{\frac{1}{60}} q T \\
& =q T K^{\frac{1}{20}} \ll Y^{1-\varepsilon},
\end{aligned}
$$

since $K \ll Y$.

II. Next, assume condition 2). We apply the mean value estimate and Halász method to $M^{2}(s, \chi), H^{5}(s, \chi)$ and $K^{2}(s, \chi) H(s, \chi)$ to get

$$
I \ll U^{2} V^{2} W^{2} Y^{-1} F \log ^{c} N,
$$

where

$$
\begin{aligned}
F=\min \{ & V^{-4}\left(M^{2}+q T\right), V^{-4} M^{2}+V^{-12} q T M^{2}, W^{-10}\left(H^{5}+q T\right), \\
& W^{-10} H^{5}+W^{-30} q T H^{5}, U^{-4} W^{-2}\left(K^{2} H+q T\right), \\
& \left.U^{-4} W^{-2} K^{2} H+U^{-12} W^{-6} q T K^{2} H\right\} .
\end{aligned}
$$

Consider the following cases:

(a) $F \leq 2 V^{-4} M^{2}, 2 W^{-10} H^{5}, 2 U^{-4} W^{-2} K^{2} H$. Then

$$
\begin{aligned}
U^{2} V^{2} W^{2} F & \ll U^{2} V^{2} W^{2} \min \left\{V^{-4} M^{2}, W^{-10} H^{5}, U^{-4} W^{-2} K^{2} H\right\} \\
& \leq U^{2} V^{2} W^{2}\left(V^{-4} M^{2}\right)^{\frac{1}{2}}\left(U^{-4} W^{-2} K^{2} H\right)^{\frac{1}{2}} \\
& =W H^{\frac{1}{2}} M K \ll Y \log ^{-7 E} N .
\end{aligned}
$$


(b) $F \leq 2 V^{-4} M^{2}, 2 W^{-10} H^{5}, F>2 U^{-4} W^{-2} K^{2} H$. Then

$U^{2} V^{2} W^{2} F$

$\ll U^{2} V^{2} W^{2} \min \left\{V^{-4} M^{2}, W^{-10} H^{5}, U^{-4} W^{-2} q T, U^{-12} W^{-6} q T K^{2} H\right\}$

$\leq U^{2} V^{2} W^{2}\left(V^{-4} M^{2}\right)^{\frac{1}{2}}\left(W^{-10} H^{5}\right)^{\frac{1}{10}}\left(U^{-4} W^{-2} q T\right)^{\frac{7}{20}}\left(U^{-12} W^{-6} q T K^{2} H\right)^{\frac{1}{20}}$

$=(q T)^{\frac{2}{5}} M H^{\frac{11}{20}} K^{\frac{1}{10}} \ll Y^{1-\varepsilon}$.

(c) $F \leq 2 V^{-4} M^{2}, F>2 W^{-10} H^{5}, F \leq 2 U^{-4} W^{-2} K^{2} H$. Then

$U^{2} V^{2} W^{2} F$

$$
\begin{aligned}
& \ll U^{2} V^{2} W^{2} \min \left\{V^{-4} M^{2}, W^{-10} q T, W^{-30} q T H^{5}, U^{-4} W^{-2} K^{2} H\right\} \\
& \leq U^{2} V^{2} W^{2}\left(V^{-4} M^{2}\right)^{\frac{1}{2}}\left(U^{-4} W^{-2} K^{2} H\right)^{\frac{1}{2}} \\
& =W H^{\frac{1}{2}} M K \ll Y \log ^{-7 E} N .
\end{aligned}
$$

(d) $F \leq 2 V^{-4} M^{2}, F>2 W^{-10} H^{5}, 2 U^{-4} W^{-2} K^{2} H$. Then

$U^{2} V^{2} W^{2} F \ll U^{2} V^{2} W^{2} \min \left\{V^{-4} M^{2}, W^{-10} q T, W^{-30} q T H^{5}, U^{-4} W^{-2} q T\right.$, $\left.U^{-12} W^{-6} q T K^{2} H\right\}$

$$
\begin{aligned}
\leq & U^{2} V^{2} W^{2}\left(V^{-4} M^{2}\right)^{\frac{1}{2}}\left(W^{-30} q T H^{5}\right)^{\frac{1}{30}}\left(U^{-4} W^{-2} q T\right)^{\frac{9}{20}} \\
& \times\left(U^{-12} W^{-6} q T K^{2} H\right)^{\frac{1}{60}} \\
= & (q T)^{\frac{1}{2}} M H^{\frac{11}{60}} K^{\frac{1}{30}} \ll Y^{1-\varepsilon} .
\end{aligned}
$$

(e) $F>2 V^{-4} M^{2}, F \leq 2 W^{-10} H^{5}, 2 U^{-4} W^{-2} K^{2} H$. Then

$U^{2} V^{2} W^{2} F$

$$
\begin{aligned}
& \ll U^{2} V^{2} W^{2} \min \left\{V^{-4} q T, V^{-12} q T M^{2}, W^{-10} H^{5}, U^{-4} W^{-2} K^{2} H\right\} \\
& \leq U^{2} V^{2} W^{2}\left(V^{-4} q T\right)^{\frac{7}{20}}\left(V^{-12} q T M^{2}\right)^{\frac{1}{20}}\left(W^{-10} H^{5}\right)^{\frac{1}{10}}\left(U^{-4} W^{-2} K^{2} H\right)^{\frac{1}{2}} \\
& =(q T)^{\frac{2}{5}} M^{\frac{1}{10}} H K \ll Y^{1-\varepsilon} .
\end{aligned}
$$

(f) $F>2 V^{-4} M^{2}, F \leq 2 W^{-10} H^{5}, F>2 U^{-4} W^{-2} K^{2} H$. Then $U^{2} V^{2} W^{2} F$

$$
\begin{aligned}
& \ll U^{2} V^{2} W^{2} \min \left\{V^{-4} q T, V^{-12} q T M^{2}, W^{-10} H^{5}, U^{-4} W^{-2} q T,\right. \\
& \left.\qquad U^{-12} W^{-6} q T K^{2} H\right\} \\
& \leq U^{2} V^{2} W^{2}\left(V^{-4} q T\right)^{\frac{7}{20}}\left(V^{-12} q T M^{2}\right)^{\frac{1}{20}}\left(W^{-10} H^{5}\right)^{\frac{1}{10}}\left(U^{-4} W^{-2} q T\right)^{\frac{1}{2}} \\
& =(q T)^{\frac{9}{10}} M^{\frac{1}{10}} H^{\frac{1}{2}} \ll Y^{1-\varepsilon} .
\end{aligned}
$$


(g) $F>2 V^{-4} M^{2}, 2 W^{-10} H^{5}, F \leq 2 U^{-4} W^{-2} K^{2} H$. Then

$$
\begin{aligned}
& U^{2} V^{2} W^{2} F \\
& \ll U^{2} V^{2} W^{2} \min \left\{V^{-4} q T, V^{-12} q T M^{2}, W^{-10} q T, W^{-30} q T H^{5},\right. \\
& \left.\quad U^{-4} W^{-2} K^{2} H\right\} \\
& \leq U^{2} V^{2} W^{2}\left(V^{-4} q T\right)^{\frac{9}{20}}\left(V^{-12} q T M^{2}\right)^{\frac{1}{60}}\left(W^{-30} q T H^{5}\right)^{\frac{1}{30}}\left(U^{-4} W^{-2} K^{2} H\right)^{\frac{1}{2}} \\
& =(q T)^{\frac{1}{2}} M^{\frac{1}{30}} H^{\frac{2}{3}} K \ll Y^{1-\varepsilon} .
\end{aligned}
$$

(h) $F>2 V^{-4} M^{2}, 2 W^{-10} H^{5}, 2 U^{-4} W^{-2} K^{2} H$. Then

$$
\begin{aligned}
U^{2} V^{2} W^{2} F & \ll U^{2} V^{2} W^{2} \min \left\{V^{-4}, V^{-12} M^{2}, W^{-10}, W^{-30} H^{5}, U^{-4} W^{-2},\right. \\
& \left.U^{-12} W^{-6} K^{2} H\right\} q T \\
& \leq U^{2} V^{2} W^{2}\left(V^{-4}\right)^{\frac{7}{20}}\left(V^{-12} M^{2}\right)^{\frac{1}{20}}\left(W^{-10}\right)^{\frac{1}{10}}\left(U^{-4} W^{-2}\right)^{\frac{1}{2}} q T \\
& =q T M^{\frac{1}{10}} \ll Y^{1-\varepsilon},
\end{aligned}
$$

since $M \ll Y^{\frac{5}{9}}$ (the latter follows from $Y^{\frac{16}{135}} \ll H$ and $M^{2} H \ll Y^{\frac{94}{81}}$ ).

III. Now, assume condition 3). We apply the mean value estimate and Halász method to $M^{2}(s, \chi), H^{4}(s, \chi)$ and $K^{3}(s, \chi)$ to get

$$
I \ll U^{2} V^{2} W^{2} Y^{-1} F \log ^{c} N,
$$

where

$$
\begin{aligned}
F=\min \left\{V^{-4}\left(M^{2}+q T\right), V^{-4} M^{2}+V^{-12} q T M^{2}, W^{-8}\left(H^{4}+q T\right),\right. \\
\\
\left.W^{-8} H^{4}+W^{-24} q T H^{4}, U^{-6}\left(K^{3}+q T\right), U^{-6} K^{3}+U^{-18} q T K^{3}\right\} .
\end{aligned}
$$

Consider the following cases:

(a) $F \leq 2 V^{-4} M^{2}, 2 W^{-8} H^{4}, 2 U^{-6} K^{3}$. Then

$$
\begin{aligned}
U^{2} V^{2} W^{2} F & \ll U^{2} V^{2} W^{2} \min \left\{V^{-4} M^{2}, W^{-8} H^{4}, U^{-6} K^{3}\right\} \\
& \leq U^{2} V^{2} W^{2}\left(V^{-4} M^{2}\right)^{\frac{1}{2}}\left(W^{-8} H^{4}\right)^{\frac{1}{6}}\left(U^{-6} K^{3}\right)^{\frac{1}{3}} \\
& =W^{\frac{2}{3}} H^{\frac{2}{3}} M K \ll Y \log ^{-7 E} N .
\end{aligned}
$$

(b) $F \leq 2 V^{-4} M^{2}, 2 W^{-8} H^{4}, F>2 U^{-6} K^{3}$. Then

$$
\begin{aligned}
U^{2} V^{2} W^{2} F & \ll U^{2} V^{2} W^{2} \min \left\{V^{-4} M^{2}, W^{-8} H^{4}, U^{-6} q T, U^{-18} q T K^{3}\right\} \\
& \leq U^{2} V^{2} W^{2}\left(V^{-4} M^{2}\right)^{\frac{1}{2}}\left(W^{-8} H^{4}\right)^{\frac{1}{4}}\left(U^{-6} q T\right)^{\frac{5}{24}}\left(U^{-18} q T K^{3}\right)^{\frac{1}{24}} \\
& =(q T)^{\frac{1}{4}} M H K^{\frac{1}{8}} \ll Y^{1-\varepsilon} .
\end{aligned}
$$


(c) $F \leq 2 V^{-4} M^{2}, F>2 W^{-8} H^{4}, F \leq 2 U^{-6} K^{3}$. Then

$$
\begin{aligned}
U^{2} V^{2} W^{2} F & \ll U^{2} V^{2} W^{2} \min \left\{V^{-4} M^{2}, W^{-8} q T, W^{-24} q T H^{4}, U^{-6} K^{3}\right\} \\
& \leq U^{2} V^{2} W^{2}\left(V^{-4} M^{2}\right)^{\frac{1}{2}}\left(W^{-8} q T\right)^{\frac{1}{8}}\left(W^{-24} q T H^{4}\right)^{\frac{1}{24}}\left(U^{-6} K^{3}\right)^{\frac{1}{3}} \\
& =(q T)^{\frac{1}{6}} M K H^{\frac{1}{6}} \ll Y^{1-\varepsilon} . \\
(\mathrm{d}) F \leq 2 V^{-4} M^{2}, F>2 W^{-8} H^{4}, 2 U^{-6} K^{3} . \text { Then } & \\
U^{2} V^{2} W^{2} F & \ll U^{2} V^{2} W^{2} \min \left\{V^{-4} M^{2}, W^{-8} q T, W^{-24} q T H^{4},\right. \\
& \left.U^{-6} q T, U^{-18} q T K^{3}\right\} \\
& \leq U^{2} V^{2} W^{2}\left(V^{-4} M^{2}\right)^{\frac{1}{2}}\left(W^{-8} q T\right)^{\frac{1}{8}}\left(W^{-24} q T H^{4}\right)^{\frac{1}{24}}\left(U^{-6} q T\right)^{\frac{1}{3}} \\
& =(q T)^{\frac{1}{2}} M H^{\frac{1}{6}} \ll Y^{1-\varepsilon} .
\end{aligned}
$$

(e) $F>2 V^{-4} M^{2}, F \leq 2 W^{-8} H^{4}, 2 U^{-6} K^{3}$. Then

$$
\begin{aligned}
U^{2} V^{2} W^{2} F & \ll U^{2} V^{2} W^{2} \min \left\{V^{-4} q T, V^{-12} q T M^{2}, W^{-8} H^{4}, U^{-6} K^{3}\right\} \\
& \leq U^{2} V^{2} W^{2}\left(V^{-4} q T\right)^{\frac{3}{8}}\left(V^{-12} q T M^{2}\right)^{\frac{1}{24}}\left(W^{-8} H^{4}\right)^{\frac{1}{4}}\left(U^{-6} K^{3}\right)^{\frac{1}{3}} \\
& =(q T)^{\frac{5}{12}} M^{\frac{1}{12}} H K \ll Y^{1-\varepsilon} .
\end{aligned}
$$

(f) $F>2 V^{-4} M^{2}, F \leq 2 W^{-8} H^{4}, F>2 U^{-6} K^{3}$. Then

$$
\begin{aligned}
U^{2} V^{2} W^{2} F \ll & U^{2} V^{2} W^{2} \min \left\{V^{-4} q T, V^{-12} q T M^{2}, W^{-8} H^{4},\right. \\
& \left.U^{-6} q T, U^{-18} q T K^{3}\right\} \\
\leq & U^{2} V^{2} W^{2}\left(V^{-4} q T\right)^{\frac{1}{2}}\left(W^{-8} H^{4}\right)^{\frac{1}{4}}\left(U^{-6} q T\right)^{\frac{5}{24}}\left(U^{-18} q T K^{3}\right)^{\frac{1}{24}} \\
= & (q T)^{\frac{3}{4}} H K^{\frac{1}{8}} \ll Y^{1-\varepsilon} .
\end{aligned}
$$

(g) $F>2 V^{-4} M^{2}, 2 W^{-8} H^{4}, F \leq 2 U^{-6} K^{3}$. Then

$$
\begin{gathered}
U^{2} V^{2} W^{2} F \ll U^{2} V^{2} W^{2} \min \left\{V^{-4} q T, V^{-12} q T M^{2}, W^{-8} q T,\right. \\
\left.W^{-24} q T H^{4}, U^{-6} K^{3}\right\}
\end{gathered}
$$$$
\leq U^{2} V^{2} W^{2}\left(V^{-4} q T\right)^{\frac{1}{2}}\left(W^{-8} q T\right)^{\frac{1}{8}}\left(W^{-24} q T H^{4}\right)^{\frac{1}{24}}\left(U^{-6} K^{3}\right)^{\frac{1}{3}}
$$$$
=(q T)^{\frac{2}{3}} H^{\frac{1}{6}} K \ll Y^{1-\varepsilon} \text {. }
$$

(h) $F>2 V^{-4} M^{2}, 2 W^{-8} H^{4}, 2 U^{-6} K^{3}$. Then

$$
\begin{aligned}
U^{2} V^{2} W^{2} F & \ll U^{2} V^{2} W^{2} \min \left\{V^{-4}, V^{-12} M^{2}, W^{-8}, W^{-24} H^{4}, U^{-6},\right. \\
& \left.U^{-18} K^{3}\right\} q T \\
\leq & U^{2} V^{2} W^{2}\left(V^{-4}\right)^{\frac{1}{2}}\left(W^{-8}\right)^{\frac{1}{4}}\left(U^{-6}\right)^{\frac{5}{24}}\left(U^{-18} K^{3}\right)^{\frac{1}{24}} q T \\
& =q T K^{\frac{1}{8}} \ll Y^{1-\varepsilon},
\end{aligned}
$$

since $Y^{\frac{5}{9}} \ll M H$ (the latter follows from $Y^{\frac{17}{90}} \ll H$ and $\left.Y^{\frac{85}{198}} \ll M\right)$. 
IV. Lastly, we assume condition 4). We apply the mean value estimate and Halász method to $M^{2}(s, \chi), H^{4}(s, \chi)$ and $K^{2}(s, \chi) H(s, \chi)$ to get

$$
I \ll U^{2} V^{2} W^{2} Y^{-1} F \log ^{c} N,
$$

where

$$
\begin{aligned}
F=\min \{ & V^{-4}\left(M^{2}+q T\right), V^{-4} M^{2}+V^{-12} q T M^{2}, W^{-8}\left(H^{4}+q T\right), \\
& W^{-8} H^{4}+W^{-24} q T H^{4}, U^{-4} W^{-2}\left(K^{2} H+q T\right), \\
& \left.U^{-4} W^{-2} K^{2} H+U^{-12} W^{-6} q T K^{2} H\right\} .
\end{aligned}
$$

Consider the following cases:

(a) $F \leq 2 V^{-4} M^{2}, 2 W^{-8} H^{4}, 2 U^{-4} W^{-2} K^{2} H$. Then

$$
\begin{aligned}
U^{2} V^{2} W^{2} F & \ll U^{2} V^{2} W^{2} \min \left\{V^{-4} M^{2}, W^{-8} H^{4}, U^{-4} W^{-2} K^{2} H\right\} \\
& \leq U^{2} V^{2} W^{2}\left(V^{-4} M^{2}\right)^{\frac{1}{2}}\left(U^{-4} W^{-2} K^{2} H\right)^{\frac{1}{2}} \\
& =W H^{\frac{1}{2}} M K \ll Y \log ^{-7 E} N .
\end{aligned}
$$

(b) $F \leq 2 V^{-4} M^{2}, 2 W^{-8} H^{4}, F>2 U^{-4} W^{-2} K^{2} H$. Then

$$
\begin{aligned}
& U^{2} V^{2} W^{2} F \\
& \quad \ll U^{2} V^{2} W^{2} \min \left\{V^{-4} M^{2}, W^{-8} H^{4}, U^{-4} W^{-2} q T, U^{-12} W^{-6} q T K^{2} H\right\} \\
& \quad \leq U^{2} V^{2} W^{2}\left(V^{-4} M^{2}\right)^{\frac{1}{2}}\left(W^{-8} H^{4}\right)^{\frac{1}{8}}\left(U^{-4} W^{-2} q T\right)^{\frac{5}{16}}\left(U^{-12} W^{-6} q T K^{2} H\right)^{\frac{1}{16}} \\
& \quad=(q T)^{\frac{3}{8}} M H^{\frac{9}{16}} K^{\frac{1}{8}} \ll Y^{1-\varepsilon} .
\end{aligned}
$$

(c) $F \leq 2 V^{-4} M^{2}, F>2 W^{-8} H^{4}, F \leq 2 U^{-4} W^{-2} K^{2} H$. Then

$$
\begin{aligned}
U^{2} V^{2} W^{2} F & \ll U^{2} V^{2} W^{2} \min \left\{V^{-4} M^{2}, W^{-8} q T, W^{-24} q T H^{4}, U^{-4} W^{-2} K^{2} H\right\} \\
& \leq U^{2} V^{2} W^{2}\left(V^{-4} M^{2}\right)^{\frac{1}{2}}\left(U^{-4} W^{-2} K^{2} H\right)^{\frac{1}{2}} \\
& =W H^{\frac{1}{2}} M K \ll Y \log ^{-7 E} N .
\end{aligned}
$$

(d) $F \leq 2 V^{-4} M^{2}, F>2 W^{-8} H^{4}, 2 U^{-4} W^{-2} K^{2} H$. Then

$$
\begin{aligned}
U^{2} V^{2} W^{2} F \ll & U^{2} V^{2} W^{2} \min \left\{V^{-4} M^{2}, W^{-8} q T, W^{-24} q T H^{4}, U^{-4} W^{-2} q T,\right. \\
& \left.U^{-12} W^{-6} q T K^{2} H\right\} \\
\leq & U^{2} V^{2} W^{2}\left(V^{-4} M^{2}\right)^{\frac{1}{2}}\left(W^{-24} q T H^{4}\right)^{\frac{1}{24}}\left(U^{-4} W^{-2} q T\right)^{\frac{7}{16}} \\
& \times\left(U^{-12} W^{-6} q T K^{2} H\right)^{\frac{1}{48}} \\
= & (q T)^{\frac{1}{2}} M H^{\frac{3}{16}} K^{\frac{1}{24}} \ll Y^{1-\varepsilon} .
\end{aligned}
$$


(e) $F>2 V^{-4} M^{2}, F \leq 2 W^{-8} H^{4}, 2 U^{-4} W^{-2} K^{2} H$. Then $U^{2} V^{2} W^{2} F$

$$
\begin{aligned}
& \ll U^{2} V^{2} W^{2} \min \left\{V^{-4} q T, V^{-12} q T M^{2}, W^{-8} H^{4}, U^{-4} W^{-2} K^{2} H\right\} \\
& \leq U^{2} V^{2} W^{2}\left(V^{-4} q T\right)^{\frac{5}{16}}\left(V^{-12} q T M^{2}\right)^{\frac{1}{16}}\left(W^{-8} H^{4}\right)^{\frac{1}{8}}\left(U^{-4} W^{-2} K^{2} H\right)^{\frac{1}{2}} \\
& =(q T)^{\frac{3}{8}} M^{\frac{1}{8}} H K \ll Y^{1-\varepsilon} .
\end{aligned}
$$

(f) $F>2 V^{-4} M^{2}, F \leq 2 W^{-8} H^{4}, F>2 U^{-4} W^{-2} K^{2} H$. Then $U^{2} V^{2} W^{2} F$

$$
\begin{aligned}
& \ll U^{2} V^{2} W^{2} \min \left\{V^{-4} q T, V^{-12} q T M^{2}, W^{-8} H^{4}, U^{-4} W^{-2} q T,\right. \\
& \left.\qquad U^{-12} W^{-6} q T K^{2} H\right\} \\
& \leq U^{2} V^{2} W^{2}\left(V^{-4} q T\right)^{\frac{5}{16}}\left(V^{-12} q T M^{2}\right)^{\frac{1}{16}}\left(W^{-8} H^{4}\right)^{\frac{1}{8}}\left(U^{-4} W^{-2} q T\right)^{\frac{1}{2}} \\
& =(q T)^{\frac{7}{8}} M^{\frac{1}{8}} H^{\frac{1}{2}} \ll Y^{1-\varepsilon} .
\end{aligned}
$$

(g) $F>2 V^{-4} M^{2}, 2 W^{-8} H^{4}, F \leq 2 U^{-4} W^{-2} K^{2} H$. Then

$$
\begin{aligned}
& U^{2} V^{2} W^{2} F \\
& \ll U^{2} V^{2} W^{2} \min \left\{V^{-4} q T, V^{-12} q T M^{2}, W^{-8} q T, W^{-24} q T H^{4},\right. \\
& \left.U^{-4} W^{-2} K^{2} H\right\} \\
& \leq U^{2} V^{2} W^{2}\left(V^{-4} q T\right)^{\frac{7}{16}}\left(V^{-12} q T M^{2}\right)^{\frac{1}{48}}\left(W^{-24} q T H^{4}\right)^{\frac{1}{24}}\left(U^{-4} W^{-2} K^{2} H\right)^{\frac{1}{2}} \\
& =(q T)^{\frac{1}{2}} M^{\frac{1}{24}} H^{\frac{2}{3}} K \ll Y^{1-\varepsilon} . \\
& (\mathrm{h}) F>2 V^{-4} M^{2}, 2 W^{-8} H^{4}, 2 U^{-4} W^{-2} K^{2} H . \text { Then } \\
& U^{2} V^{2} W^{2} F \ll U^{2} V^{2} W^{2} \min \left\{V^{-4}, V^{-12} M^{2}, W^{-8}, W^{-24} H^{4}, U^{-4} W^{-2},\right. \\
& \left.\quad U^{-12} W^{-6} K^{2} H\right\} q T \\
& \leq U^{2} V^{2} W^{2}\left(V^{-4}\right)^{\frac{5}{16}}\left(V^{-12} M^{2}\right)^{\frac{1}{16}}\left(W^{-8}\right)^{\frac{1}{8}}\left(U^{-4} W^{-2}\right)^{\frac{1}{2}} q T \\
& =q T M^{\frac{1}{8}} \ll Y^{1-\varepsilon} .
\end{aligned}
$$

Combining the above, we obtain (9). Hence, Lemma 9 follows.

LEMma 10. Under the assumption of Lemma 7, suppose that $M$ and $H$ lie in one of the following regions:
(i) $\quad Y^{\frac{11}{27}} \ll M \ll Y^{\frac{34}{81}}, \quad M^{-\frac{23}{8}} Y^{\frac{17}{12}} \ll H \ll M^{-\frac{1}{4}} Y^{\frac{25}{72}} ;$
(ii) $\quad Y^{\frac{34}{81}} \ll M \ll Y^{\frac{49}{114}}, \quad M^{-\frac{29}{10}} Y^{\frac{17}{12}} \ll H \ll M^{-\frac{1}{4}} Y^{\frac{25}{72}}$;
(iii) $\quad Y^{\frac{49}{114}} \ll M \ll Y^{\frac{4}{9}}, \quad M^{-\frac{29}{10}} Y^{\frac{17}{12}} \ll H \ll M^{\frac{1}{7}} Y^{\frac{4}{21}}$;
(iv) $\quad Y^{\frac{4}{9}} \ll M \ll Y^{\frac{701}{1566}}, \quad M^{-\frac{29}{10}} Y^{\frac{17}{12}} \ll H \ll M^{-\frac{1}{5}} Y^{\frac{3}{10}}$;
(v) $\quad Y^{\frac{4}{9}} \ll M \ll Y^{\frac{701}{1566}}, \quad M^{-\frac{6}{5}} Y^{\frac{34}{45}} \ll H \ll M^{\frac{1}{7}} Y^{\frac{4}{21}}$; 
(vi) $\quad Y^{\frac{701}{1566}} \ll M \ll Y^{\frac{41}{90}}, \quad Y^{\frac{16}{135}} \ll H \ll M^{-\frac{1}{5}} Y^{\frac{3}{10}} ;$

(vii) $\quad Y^{\frac{701}{1566}} \ll M \ll Y^{\frac{41}{90}}, \quad M^{-\frac{6}{5}} Y^{\frac{34}{45}} \ll H \ll M^{\frac{1}{7}} Y^{\frac{4}{21}}$;

(viii) $\quad Y^{\frac{41}{90}} \ll M \ll Y^{\frac{17}{36}}, \quad Y^{\frac{16}{135}} \ll H \ll M^{\frac{1}{7}} Y^{\frac{4}{21}}$;

(ix) $\quad Y^{\frac{17}{36}} \ll M \ll Y^{\frac{16}{33}}, \quad Y^{\frac{16}{135}} \ll H \ll M^{-1} Y^{\frac{46}{63}} ;$

(x) $\quad Y^{\frac{16}{33}} \ll M \ll Y^{\frac{307}{630}}, \quad Y^{\frac{16}{135}} \ll H \ll M^{-\frac{58}{9}} Y^{\frac{89}{27}} ;$

(xi) $\quad Y^{\frac{16}{33}} \ll M \ll Y^{\frac{307}{630}}, \quad Y^{\frac{17}{99}} \ll H \ll M^{-1} Y^{\frac{46}{63}} ;$

(xii) $\quad Y^{\frac{307}{630}} \ll M \ll Y^{\frac{281}{570}}, \quad Y^{\frac{16}{135}} \ll H \ll M^{-\frac{58}{9}} Y^{\frac{89}{27}}$;

(xiii) $\quad Y^{\frac{307}{630}} \ll M \ll Y^{\frac{281}{570}}, \quad Y^{\frac{17}{99}} \ll H \ll M^{-6} Y^{\frac{19}{6}}$;

(xiv) $\quad Y^{\frac{281}{570}} \ll M \ll Y^{\frac{429}{870}}, \quad Y^{\frac{16}{135}} \ll H \ll M^{-\frac{58}{9}} Y^{\frac{89}{27}} ;$

(xv) $\quad Y^{\frac{429}{870}} \ll M \ll Y^{\frac{1}{2}}, \quad Y^{\frac{17}{99}} \ll H \ll M^{-1} Y^{\frac{40}{57}}$.

Then (8) holds for $T_{0} \leq T \leq Y$.

Pr o of. In the regions:

$$
\begin{array}{ll}
Y^{\frac{119}{261}} \ll M \ll Y^{\frac{17}{36}}, & M^{-\frac{12}{11}} Y^{\frac{68}{99}} \ll H \ll M^{\frac{1}{19}} Y^{\frac{35}{171}} ; \\
Y^{\frac{17}{36}} \ll M \ll Y^{\frac{281}{570}}, & Y^{\frac{17}{99}} \ll H \ll M^{-1} Y^{\frac{40}{57}} ; \\
Y^{\frac{429}{870}} \ll M \ll Y^{\frac{1}{2}}, & Y^{\frac{17}{99}} \ll H \ll M^{-1} Y^{\frac{40}{57}},
\end{array}
$$

we apply Lemma 9 with condition 1).

In the regions:

$$
\begin{array}{ll}
Y^{\frac{34}{81}} \ll M \ll Y^{\frac{701}{1566}}, & M^{-\frac{29}{10}} Y^{\frac{17}{12}} \ll H \ll M^{-\frac{1}{5}} Y^{\frac{3}{10}} ; \\
Y^{\frac{701}{1566}} \ll M \ll Y^{\frac{119}{261}}, & Y^{\frac{16}{135}} \ll H \ll M^{-\frac{1}{5}} Y^{\frac{3}{10}} ; \\
Y^{\frac{119}{261}} \ll M \ll Y^{\frac{17}{36}}, & Y^{\frac{16}{135}} \ll H \ll M^{-\frac{12}{11}} Y^{\frac{68}{99}} ; \\
Y^{\frac{17}{36}} \ll M \ll Y^{\frac{16}{33}}, & Y^{\frac{16}{135}} \ll H \ll Y^{\frac{17}{99}} ; \\
Y^{\frac{16}{33}} \ll M \ll Y^{\frac{429}{870}}, & Y^{\frac{16}{135}} \ll H \ll M^{-\frac{58}{9}} Y^{\frac{89}{27}},
\end{array}
$$

we apply Lemma 9 with condition 2).

In the regions:

$$
\begin{array}{ll}
Y^{\frac{49}{114}} \ll M \ll Y^{\frac{41}{90}}, & M^{-\frac{6}{5}} Y^{\frac{34}{45}} \ll H \ll M^{\frac{1}{7}} Y^{\frac{4}{21}} ; \\
Y^{\frac{41}{90}} \ll M \ll Y^{\frac{119}{261}}, & M^{-\frac{1}{5}} Y^{\frac{3}{10}} \ll H \ll M^{\frac{1}{7}} Y^{\frac{4}{21}} ; \\
Y^{\frac{119}{261}} \ll M \ll Y^{\frac{17}{36}}, & M^{\frac{1}{19}} Y^{\frac{35}{171}} \ll H \ll M^{\frac{1}{7}} Y^{\frac{4}{21}} ; \\
Y^{\frac{17}{36}} \ll M \ll Y^{\frac{307}{630}}, & M^{-1} Y^{\frac{40}{57}} \ll H \ll M^{-1} Y^{\frac{46}{63}} ; \\
Y^{\frac{307}{630}} \ll M \ll Y^{\frac{281}{570}}, & M^{-1} Y^{\frac{40}{57}} \ll H \ll M^{-6} Y^{\frac{19}{6}},
\end{array}
$$

we apply Lemma 9 with condition 3 ). 
In the regions:

$$
\begin{array}{ll}
Y^{\frac{11}{27}} \ll M \ll Y^{\frac{34}{81}}, & M^{-\frac{23}{8}} Y^{\frac{17}{12}} \ll H \ll M^{-\frac{1}{4}} Y^{\frac{25}{72}} ; \\
Y^{\frac{34}{81}} \ll M \ll Y^{\frac{49}{114}}, & M^{-\frac{1}{5}} Y^{\frac{3}{10}} \ll H \ll M^{-\frac{1}{4}} Y^{\frac{25}{72}} ; \\
Y^{\frac{49}{114}} \ll M \ll Y^{\frac{4}{9}}, & M^{-\frac{1}{5}} Y^{\frac{3}{10}} \ll H \ll M^{-\frac{6}{5}} Y^{\frac{34}{45}},
\end{array}
$$

we apply Lemma 9 with condition 4).

Putting together the above regions, we get Lemma 10.

\section{Mean value estimate (III)}

LEMMA 11. Under the assumption of Lemma 7, suppose that $M$ and $H$ lie in one of the following regions:

$$
\begin{array}{lll}
\text { (i) } & Y^{\frac{34}{99}} \ll M \ll Y^{\frac{1649}{4752}}, & M^{-2} Y^{\frac{85}{99}} \ll H \ll M^{-\frac{58}{49}} Y^{\frac{85}{147}} ; \\
\text { (ii) } & Y^{\frac{1649}{4752}} \ll M \ll Y^{\frac{1823}{4725}}, & M^{-\frac{70}{59}} Y^{\frac{34}{59}} \ll H \ll M^{-\frac{58}{49}} Y^{\frac{85}{147}} ; \\
\text { (iii) } & Y^{\frac{1823}{4725}} \ll M \ll Y^{\frac{3041}{7830}}, & Y^{\frac{16}{135}} \ll H \ll M^{-\frac{58}{49}} Y^{\frac{85}{147}} ; \\
\text { (iv) } & Y^{\frac{11}{27}} \ll M \ll Y^{\frac{49}{114}}, & M^{-\frac{29}{19}} Y^{\frac{89}{114}} \ll H \ll M^{-\frac{35}{23}} Y^{\frac{18}{23}}
\end{array}
$$

Then (8) holds for $T_{0} \leq T \leq Y$.

Proof. First we show that (9) holds for $T=1 / \eta=Y /(q Q)$, providing $M$ and $H$ satisfy the following conditions:

$$
\begin{gathered}
M H \ll Y^{\frac{113}{198}}, \quad M^{\frac{35}{23}} H \ll Y^{\frac{18}{23}}, \quad Y^{\frac{85}{99}} \ll M^{2} H, \\
H^{5} / M \ll Y^{\frac{11}{18}}, \quad Y^{\frac{34}{59}} \ll M^{\frac{70}{59}} H, \quad Y^{\frac{16}{135}} \ll H .
\end{gathered}
$$

We apply the mean value estimate and Halász method to $M^{2}(s, \chi)$ $\times H(s, \chi), H^{6}(s, \chi)$ and $K^{2}(s, \chi)$ to get

$$
I \ll U^{2} V^{2} W^{2} Y^{-1} F \log ^{c} N,
$$

where

$$
\begin{aligned}
F=\min \{ & V^{-4} W^{-2}\left(M^{2} H+q T\right), V^{-4} W^{-2} M^{2} H+V^{-12} W^{-6} q T M^{2} H, \\
& W^{-12}\left(H^{6}+q T\right), W^{-12} H^{6}+W^{-36} q T H^{6}, U^{-4}\left(K^{2}+q T\right), \\
& \left.U^{-4} K^{2}+U^{-12} q T K^{2}\right\} .
\end{aligned}
$$

Consider the following cases:

(a) $F \leq 2 V^{-4} W^{-2} M^{2} H, 2 W^{-12} H^{6}, 2 U^{-4} K^{2}$. Then

$$
\begin{aligned}
U^{2} V^{2} W^{2} F & \ll U^{2} V^{2} W^{2} \min \left\{V^{-4} W^{-2} M^{2} H, W^{-12} H^{6}, U^{-4} K^{2}\right\} \\
& \leq U^{2} V^{2} W^{2}\left(V^{-4} W^{-2} M^{2} H\right)^{\frac{1}{2}}\left(U^{-4} K^{2}\right)^{\frac{1}{2}} \\
& =W H^{\frac{1}{2}} M K \ll Y \log ^{-7 E} N .
\end{aligned}
$$


(b) $F \leq 2 V^{-4} W^{-2} M^{2} H, 2 W^{-12} H^{6}, F>2 U^{-4} K^{2}$. Then $U^{2} V^{2} W^{2} F$

$$
\begin{aligned}
& \ll U^{2} V^{2} W^{2} \min \left\{V^{-4} W^{-2} M^{2} H, W^{-12} H^{6}, U^{-4} q T, U^{-12} q T K^{2}\right\} \\
& \leq U^{2} V^{2} W^{2}\left(V^{-4} W^{-2} M^{2} H\right)^{\frac{1}{2}}\left(W^{-12} H^{6}\right)^{\frac{1}{12}}\left(U^{-4} q T\right)^{\frac{3}{8}}\left(U^{-12} q T K^{2}\right)^{\frac{1}{24}} \\
& =(q T)^{\frac{5}{12}} M H K^{\frac{1}{12}} \ll Y^{1-\varepsilon} .
\end{aligned}
$$

(c) $F \leq 2 V^{-4} W^{-2} M^{2} H, F>2 W^{-12} H^{6}, F \leq 2 U^{-4} K^{2}$. Then

$$
\begin{aligned}
U^{2} V^{2} W^{2} F & \ll U^{2} V^{2} W^{2} \min \left\{V^{-4} W^{-2} M^{2} H, W^{-12} q T, W^{-36} q T H^{6}, U^{-4} K^{2}\right\} \\
& \leq U^{2} V^{2} W^{2}\left(V^{-4} W^{-2} M^{2} H\right)^{\frac{1}{2}}\left(U^{-4} K^{2}\right)^{\frac{1}{2}} \\
& =W H^{\frac{1}{2}} M K \ll Y \log ^{-7 E} N .
\end{aligned}
$$

(d) $F \leq 2 V^{-4} W^{-2} M^{2} H, F>2 W^{-12} H^{6}, 2 U^{-4} K^{2}$. Then

$$
\begin{aligned}
& U^{2} V^{2} W^{2} F \\
& \quad \ll U^{2} V^{2} W^{2} \min \left\{V^{-4} W^{-2} M^{2} H, W^{-12} q T, W^{-36} q T H^{6}, U^{-4} q T,\right.
\end{aligned}
$$

$$
\left.U^{-12} q T K^{2}\right\}
$$

$\leq U^{2} V^{2} W^{2}\left(V^{-4} W^{-2} M^{2} H\right)^{\frac{1}{2}}\left(W^{-36} q T H^{6}\right)^{\frac{1}{36}}\left(U^{-4} q T\right)^{\frac{11}{24}}\left(U^{-12} q T K^{2}\right)^{\frac{1}{72}}$

$=(q T)^{\frac{1}{2}} M H^{\frac{2}{3}} K^{\frac{1}{36}} \ll Y^{1-\varepsilon}$.

(e) $F>2 V^{-4} W^{-2} M^{2} H, F \leq 2 W^{-12} H^{6}, 2 U^{-4} K^{2}$. Then

$$
\begin{aligned}
& U^{2} V^{2} W^{2} F \\
& \quad \ll U^{2} V^{2} W^{2} \min \left\{V^{-4} W^{-2} q T, V^{-12} W^{-6} q T M^{2} H, W^{-12} H^{6}, U^{-4} K^{2}\right\} \\
& \quad \leq U^{2} V^{2} W^{2}\left(V^{-4} W^{-2} q T\right)^{\frac{3}{8}}\left(V^{-12} W^{-6} q T M^{2} H\right)^{\frac{1}{24}}\left(W^{-12} H^{6}\right)^{\frac{1}{12}}\left(U^{-4} K^{2}\right)^{\frac{1}{2}} \\
& =(q T)^{\frac{5}{12}} M^{\frac{1}{12}} H^{\frac{13}{24}} K \ll Y^{1-\varepsilon} .
\end{aligned}
$$

(f) $F>2 V^{-4} W^{-2} M^{2} H, F \leq 2 W^{-12} H^{6}, F>2 U^{-4} K^{2}$. Then

$$
U^{2} V^{2} W^{2} F
$$

$$
\begin{aligned}
& \ll U^{2} V^{2} W^{2} \min \left\{V^{-4} W^{-2} q T, V^{-12} W^{-6} q T M^{2} H, W^{-12} H^{6},\right. \\
& \left.\qquad U^{-4} q T, U^{-12} q T K^{2}\right\} \\
& \leq U^{2} V^{2} W^{2}\left(V^{-4} W^{-2} q T\right)^{\frac{1}{2}}\left(W^{-12} H^{6}\right)^{\frac{1}{12}}\left(U^{-4} q T\right)^{\frac{3}{8}}\left(U^{-12} q T K^{2}\right)^{\frac{1}{24}} \\
& =(q T)^{\frac{11}{12}} H^{\frac{1}{2}} K^{\frac{1}{12}} \ll Y^{1-\varepsilon} .
\end{aligned}
$$


(g) $F>2 V^{-4} W^{-2} M^{2} H, 2 W^{-12} H^{6}, F \leq 2 U^{-4} K^{2}$. Then

$$
\begin{aligned}
U^{2} V^{2} W^{2} F \ll & U^{2} V^{2} W^{2} \min \left\{V^{-4} W^{-2} q T, V^{-12} W^{-6} q T M^{2} H, W^{-12} q T,\right. \\
& \left.W^{-36} q T H^{6}, U^{-4} K^{2}\right\} \\
\leq & U^{2} V^{2} W^{2}\left(V^{-4} W^{-2} q T\right)^{\frac{11}{24}}\left(V^{-12} W^{-6} q T M^{2} H\right)^{\frac{1}{72}} \\
& \times\left(W^{-36} q T H^{6}\right)^{\frac{1}{36}}\left(U^{-4} K^{2}\right)^{\frac{1}{2}} \\
= & (q T)^{\frac{1}{2}} M^{\frac{1}{36}} H^{\frac{13}{72}} K \ll Y^{1-\varepsilon} .
\end{aligned}
$$

(h) $F>2 V^{-4} W^{-2} M^{2} H, 2 W^{-12} H^{6}, 2 U^{-4} K^{2}$. Then

$$
\begin{aligned}
U^{2} V^{2} W^{2} F \ll & U^{2} V^{2} W^{2} \min \left\{V^{-4} W^{-2}, V^{-12} W^{-6} M^{2} H, W^{-12},\right. \\
& \left.W^{-36} H^{6}, U^{-4}, U^{-12} K^{2}\right\} q T \\
\leq & U^{2} V^{2} W^{2}\left(V^{-4} W^{-2}\right)^{\frac{1}{2}}\left(W^{-12}\right)^{\frac{1}{12}}\left(U^{-4}\right)^{\frac{3}{8}}\left(U^{-12} K^{2}\right)^{\frac{1}{24}} q T \\
& =q T K^{\frac{1}{12}} \ll Y^{1-\varepsilon},
\end{aligned}
$$

since $Y^{\frac{1}{3}} \ll M H$ (the latter follows from $Y^{\frac{85}{99}} \ll M^{2} H$ and $Y^{\frac{16}{135}} \ll H$ ).

In every region, our conditions are satisfied. So the proof of Lemma 11 is complete.

Lemma 12. Under the assumption of Lemma 7, suppose that $M$ and $H$ lie in one of the following regions:

$$
\begin{array}{ll}
Y^{\frac{3397}{7830}} \ll M \ll Y^{\frac{4}{9}}, & M^{-\frac{35}{12}} Y^{\frac{17}{12}} \ll H \ll M^{-\frac{29}{10}} Y^{\frac{17}{12}} ; \\
Y^{\frac{211}{432}} \ll M \ll Y^{\frac{281}{570}}, & M^{-\frac{58}{9}} Y^{\frac{89}{27}} \ll H \ll M^{-\frac{70}{11}} Y^{\frac{36}{11}} .
\end{array}
$$

Then (8) holds for $T_{0} \leq T \leq Y$.

Proof. First we show that (9) holds for $T=1 / \eta=Y /(q Q)$, providing that $M$ and $H$ satisfy the following conditions:

$$
\begin{gathered}
M^{2} H \ll Y^{\frac{113}{99}}, \quad M^{\frac{70}{11}} H \ll Y^{\frac{36}{11}}, \quad Y^{\frac{85}{198}} \ll M, \\
M^{\frac{1}{6}} H \ll Y^{\frac{29}{108}}, \quad Y^{\frac{17}{12}} \ll M^{\frac{35}{12}} H, \quad M \ll Y^{\frac{1}{2}} .
\end{gathered}
$$

We apply the mean value estimate and Halász method to $M^{2}(s, \chi)$, $H^{6}(s, \chi)$ and $K^{2}(s, \chi) H(s, \chi)$ to get

$$
I \ll U^{2} V^{2} W^{2} Y^{-1} F \log ^{c} N,
$$

where

$$
\begin{aligned}
F=\min \{ & V^{-4}\left(M^{2}+q T\right), V^{-4} M^{2}+V^{-12} q T M^{2}, W^{-12}\left(H^{6}+q T\right), \\
& W^{-12} H^{6}+W^{-36} q T H^{6}, U^{-4} W^{-2}\left(K^{2} H+q T\right), \\
& \left.U^{-4} W^{-2} K^{2} H+U^{-12} W^{-6} q T K^{2} H\right\} .
\end{aligned}
$$


Consider the following cases:

(a) $F \leq 2 V^{-4} M^{2}, 2 W^{-12} H^{6}, 2 U^{-4} W^{-2} K^{2} H$. Then

$$
\begin{aligned}
U^{2} V^{2} W^{2} F & \ll U^{2} V^{2} W^{2} \min \left\{V^{-4} M^{2}, W^{-12} H^{6}, U^{-4} W^{-2} K^{2} H\right\} \\
& \leq U^{2} V^{2} W^{2}\left(V^{-4} M^{2}\right)^{\frac{1}{2}}\left(U^{-4} W^{-2} K^{2} H\right)^{\frac{1}{2}} \\
& =W H^{\frac{1}{2}} M K \ll Y \log ^{-7 E} N .
\end{aligned}
$$

(b) $F \leq 2 V^{-4} M^{2}, 2 W^{-12} H^{6}, F>2 U^{-4} W^{-2} K^{2} H$. Then

$$
\begin{aligned}
& U^{2} V^{2} W^{2} F \\
& \quad \ll U^{2} V^{2} W^{2} \min \left\{V^{-4} M^{2}, W^{-12} H^{6}, U^{-4} W^{-2} q T, U^{-12} W^{-6} q T K^{2} H\right\} \\
& \leq U^{2} V^{2} W^{2}\left(V^{-4} M^{2}\right)^{\frac{1}{2}}\left(W^{-12} H^{6}\right)^{\frac{1}{12}}\left(U^{-4} W^{-2} q T\right)^{\frac{3}{8}}\left(U^{-12} W^{-6} q T K^{2} H\right)^{\frac{1}{24}} \\
& =(q T)^{\frac{5}{12}} M H^{\frac{13}{24}} K^{\frac{1}{12}} \ll Y^{1-\varepsilon} .
\end{aligned}
$$

(c) $F \leq 2 V^{-4} M^{2}, F>2 W^{-12} H^{6}, F \leq 2 U^{-4} W^{-2} K^{2} H$. Then

$$
\begin{aligned}
U^{2} V^{2} W^{2} F \ll & U^{2} V^{2} W^{2} \min \left\{V^{-4} M^{2}, W^{-12} q T, W^{-36} q T H^{6},\right. \\
& \left.U^{-4} W^{-2} K^{2} H\right\} \\
\leq & U^{2} V^{2} W^{2}\left(V^{-4} M^{2}\right)^{\frac{1}{2}}\left(U^{-4} W^{-2} K^{2} H\right)^{\frac{1}{2}} \\
& =W H^{\frac{1}{2}} M K \ll Y \log ^{-7 E} N .
\end{aligned}
$$

(d) $F \leq 2 V^{-4} M^{2}, F>2 W^{-12} H^{6}, 2 U^{-4} W^{-2} K^{2} H$. Then

$$
\begin{aligned}
U^{2} V^{2} W^{2} F \ll & U^{2} V^{2} W^{2} \min \left\{V^{-4} M^{2}, W^{-12} q T, W^{-36} q T H^{6}, U^{-4} W^{-2} q T,\right. \\
& \left.U^{-12} W^{-6} q T K^{2} H\right\} \\
\leq & U^{2} V^{2} W^{2}\left(V^{-4} M^{2}\right)^{\frac{1}{2}}\left(W^{-36} q T H^{6}\right)^{\frac{1}{36}}\left(U^{-4} W^{-2} q T\right)^{\frac{11}{24}} \\
& \times\left(U^{-12} W^{-6} q T K^{2} H\right)^{\frac{1}{72}} \\
= & (q T)^{\frac{1}{2}} M H^{\frac{13}{72}} K^{\frac{1}{36}} \ll Y^{1-\varepsilon} .
\end{aligned}
$$

(e) $F>2 V^{-4} M^{2}, F \leq 2 W^{-12} H^{6}, 2 U^{-4} W^{-2} K^{2} H$. Then

$$
\begin{aligned}
U^{2} V^{2} & W^{2} F \\
& \ll U^{2} V^{2} W^{2} \min \left\{V^{-4} q T, V^{-12} q T M^{2}, W^{-12} H^{6}, U^{-4} W^{-2} K^{2} H\right\} \\
& \leq U^{2} V^{2} W^{2}\left(V^{-4} q T\right)^{\frac{3}{8}}\left(V^{-12} q T M^{2}\right)^{\frac{1}{24}}\left(W^{-12} H^{6}\right)^{\frac{1}{12}}\left(U^{-4} W^{-2} K^{2} H\right)^{\frac{1}{2}} \\
& =(q T)^{\frac{5}{12}} M^{\frac{1}{12}} H K \ll Y^{1-\varepsilon} .
\end{aligned}
$$


(f) $F>2 V^{-4} M^{2}, F \leq 2 W^{-12} H^{6}, F>2 U^{-4} W^{-2} K^{2} H$. Then

$$
\begin{aligned}
U^{2} V^{2} W^{2} F \ll & U^{2} V^{2} W^{2} \min \left\{V^{-4} q T, V^{-12} q T M^{2}, W^{-12} H^{6}, U^{-4} W^{-2} q T,\right. \\
& \left.U^{-12} W^{-6} q T K^{2} H\right\} \\
\leq & U^{2} V^{2} W^{2}\left(V^{-4} q T\right)^{\frac{3}{8}}\left(V^{-12} q T M^{2}\right)^{\frac{1}{24}}\left(W^{-12} H^{6}\right)^{\frac{1}{12}} \\
& \times\left(U^{-4} W^{-2} q T\right)^{\frac{1}{2}} \\
= & (q T)^{\frac{11}{12}} M^{\frac{1}{12}} H^{\frac{1}{2}} \ll Y^{1-\varepsilon} .
\end{aligned}
$$

(g) $F>2 V^{-4} M^{2}, 2 W^{-12} H^{6}, F \leq 2 U^{-4} W^{-2} K^{2} H$. Then

$$
\begin{aligned}
& U^{2} V^{2} W^{2} F \\
& \ll U^{2} V^{2} W^{2} \min \left\{V^{-4} q T, V^{-12} q T M^{2}, W^{-12} q T, W^{-36} q T H^{6},\right. \\
& \left.\quad U^{-4} W^{-2} K^{2} H\right\} \\
& \leq U^{2} V^{2} W^{2}\left(V^{-4} q T\right)^{\frac{11}{24}}\left(V^{-12} q T M^{2}\right)^{\frac{1}{72}}\left(W^{-36} q T H^{6}\right)^{\frac{1}{36}}\left(U^{-4} W^{-2} K^{2} H\right)^{\frac{1}{2}} \\
& =(q T)^{\frac{1}{2}} M^{\frac{1}{36}} H^{\frac{2}{3}} K \ll Y^{1-\varepsilon} .
\end{aligned}
$$

(h) $F>2 V^{-4} M^{2}, 2 W^{-12} H^{6}, 2 U^{-4} W^{-2} K^{2} H$. Then

$$
\begin{aligned}
U^{2} V^{2} W^{2} F & \ll U^{2} V^{2} W^{2} \min \left\{V^{-4}, V^{-12} M^{2}, W^{-12}, W^{-36} H^{6}, U^{-4} W^{-2},\right. \\
& \left.U^{-12} W^{-6} K^{2} H\right\} q T \\
& \leq U^{2} V^{2} W^{2}\left(V^{-4}\right)^{\frac{3}{8}}\left(V^{-12} M^{2}\right)^{\frac{1}{24}}\left(W^{-12}\right)^{\frac{1}{12}}\left(U^{-4} W^{-2}\right)^{\frac{1}{2}} q T \\
& =q T M^{\frac{1}{12}} \ll Y^{1-\varepsilon} .
\end{aligned}
$$

In every region, our conditions are satisfied, so the proof of Lemma 12 is complete.

Lemma 13. Assume that $P Q R K=Y, q \leq Q, \chi$ is a character $\bmod q$, $P(s, \chi), Q(s, \chi), R(s, \chi)$ and $K(s, \chi)$ are Dirichlet polynomials and $G(s, \chi)$ $=P(s, \chi) Q(s, \chi) R(s, \chi) K(s, \chi)$. Let $\eta=q Q / Y, b=1+1 / \log N, T_{0}=$ $\log \frac{E}{\delta^{2}} Y$. Assume further that for $T_{0} \leq|t| \leq 2 Y, P(b+i t, \chi) Q(b+i t, \chi) \ll$ $\log ^{-\frac{E}{\delta^{2}}} Y$ and $R(b+i t, \chi) \ll \log ^{-\frac{E}{\delta^{2}}} Y$. Moreover, assume that

$$
Y^{\frac{16}{135}} \ll R \ll Q
$$

and that $P$ and $Q$ lie in one of the following regions:

$$
Y^{\frac{41}{180}} \ll P \ll Y^{\frac{8}{33}}, \quad P^{-1} Y^{\frac{41}{90}} \ll Q \ll P
$$

$$
Y^{\frac{8}{33}} \ll P \ll Y^{\frac{37}{150}}, \quad P^{-1} Y^{\frac{41}{90}} \ll Q \ll P^{-1} Y^{\frac{16}{33}} ;
$$

(iii) $\quad Y^{\frac{37}{150}} \ll P \ll Y^{\frac{31}{99}}, \quad P^{-1} Y^{\frac{701}{1566}} \ll Q \ll P^{-1} Y^{\frac{16}{33}}$; 
(iv)

$$
\begin{array}{ll}
Y^{\frac{31}{99}} \ll P \ll Y^{\frac{859}{2610}}, & P^{-1} Y^{\frac{701}{1566}} \ll Q \ll P^{-\frac{58}{67}} Y^{\frac{89}{201}} \\
Y^{\frac{859}{2610}} \ll P \ll Y^{\frac{10}{27}}, & Y^{\frac{16}{135}} \ll Q \ll P^{-\frac{58}{67}} Y^{\frac{89}{201}} .
\end{array}
$$

Then (8) holds for $T_{0} \leq T \leq Y$.

Proof. Let $m=p q, h=r$.

(a) On applying Lemma 10 with region (vi), we see that (8) holds under the conditions

$$
P^{-1} Y^{\frac{701}{1566}} \ll Q \ll P^{-1} Y^{\frac{41}{90}}, \quad Y^{\frac{16}{135}} \ll R \ll Q \ll(P Q)^{-\frac{1}{5}} Y^{\frac{3}{10}},
$$

which can be written as

$$
P^{-1} Y^{\frac{701}{1566}} \ll Q \ll P^{-1} Y^{\frac{41}{90}}, \quad Y^{\frac{16}{135}} \ll Q \ll P^{-\frac{1}{6}} Y^{\frac{1}{4}}, \quad Y^{\frac{16}{135}} \ll R \ll Q .
$$

In the regions:

$$
\begin{array}{ll}
Y^{\frac{37}{150}} \ll P \ll Y^{\frac{859}{2610}}, & P^{-1} Y^{\frac{701}{1566}} \ll Q \ll P^{-1} Y^{\frac{41}{90}} \\
Y^{\frac{859}{2610}} \ll P \ll Y^{\frac{91}{270}}, & Y^{\frac{16}{135}} \ll Q \ll P^{-1} Y^{\frac{41}{90}},
\end{array}
$$

the above conditions on $P$ and $Q$ are satisfied.

(b) On applying Lemma 10 with region (viii), we see that (8) holds under the conditions

$$
P^{-1} Y^{\frac{41}{90}} \ll Q \ll P^{-1} Y^{\frac{17}{36}}, \quad Y^{\frac{16}{135}} \ll R \ll Q \ll(P Q)^{\frac{1}{7}} Y^{\frac{4}{21}},
$$

which can be written as

$$
P^{-1} Y^{\frac{41}{90}} \ll Q \ll P^{-1} Y^{\frac{17}{36}}, \quad Y^{\frac{16}{135}} \ll Q \ll P^{\frac{1}{6}} Y^{\frac{2}{9}}, \quad Y^{\frac{16}{135}} \ll R \ll Q .
$$

In the regions:

$$
\begin{array}{ll}
Y^{\frac{41}{180}} \ll P \ll Y^{\frac{17}{72}}, & P^{-1} Y^{\frac{41}{90}} \ll Q \ll P ; \\
Y^{\frac{17}{72}} \ll P \ll Y^{\frac{91}{270}}, & P^{-1} Y^{\frac{41}{90}} \ll Q \ll P^{-1} Y^{\frac{17}{36}} ; \\
Y^{\frac{91}{270}} \ll P \ll Y^{\frac{191}{540}}, & Y^{\frac{16}{135}} \ll Q \ll P^{-1} Y^{\frac{17}{36}},
\end{array}
$$

the above conditions on $P$ and $Q$ are satisfied.

(c) On applying Lemma 10 with region (ix), we see that (8) holds under the conditions

$$
P^{-1} Y^{\frac{17}{36}} \ll Q \ll P^{-1} Y^{\frac{16}{33}}, \quad Y^{\frac{16}{135}} \ll R \ll Q \ll(P Q)^{-1} Y^{\frac{46}{63}},
$$

which can be written as

$$
P^{-1} Y^{\frac{17}{36}} \ll Q \ll P^{-1} Y^{\frac{16}{33}}, \quad Y^{\frac{16}{135}} \ll Q \ll P^{-\frac{1}{2}} Y^{\frac{23}{63}}, \quad Y^{\frac{16}{135}} \ll R \ll Q .
$$

In the regions:

$$
\begin{array}{ll}
Y^{\frac{17}{72}} \ll P \ll Y^{\frac{8}{33}}, & P^{-1} Y^{\frac{17}{36}} \ll Q \ll P ; \\
Y^{\frac{8}{33}} \ll P \ll Y^{\frac{191}{540}}, & P^{-1} Y^{\frac{17}{36}} \ll Q \ll P^{-1} Y^{\frac{16}{33}} ; \\
Y^{\frac{191}{540}} \ll P \ll Y^{\frac{544}{1485}}, & Y^{\frac{16}{135}} \ll Q \ll P^{-1} Y^{\frac{16}{33}},
\end{array}
$$

the above conditions on $P$ and $Q$ are satisfied. 
(d) On applying Lemma 10 with regions (x), (xii) and (xiv), we see that (8) holds under the conditions

$$
P^{-1} Y^{\frac{16}{33}} \ll Q \ll P^{-1} Y^{\frac{429}{870}}, \quad Y^{\frac{16}{135}} \ll R \ll Q \ll(P Q)^{-\frac{58}{9}} Y^{\frac{89}{27}},
$$

which can be written as

$$
P^{-1} Y^{\frac{16}{33}} \ll Q \ll P^{-1} Y^{\frac{429}{870}}, \quad Y^{\frac{16}{135}} \ll Q \ll P^{-\frac{58}{67}} Y^{\frac{89}{201}}, \quad Y^{\frac{16}{135}} \ll R \ll Q .
$$

In the regions:

$$
\begin{array}{ll}
Y^{\frac{31}{99}} \ll P \ll Y^{\frac{544}{1485}}, & P^{-1} Y^{\frac{16}{33}} \ll Q \ll P^{-\frac{58}{67}} Y^{\frac{89}{201}} \\
Y^{\frac{544}{1485}} \ll P \ll Y^{\frac{10}{27}}, & Y^{\frac{16}{135}} \ll Q \ll P^{-\frac{58}{67}} Y^{\frac{89}{201}},
\end{array}
$$

the above conditions on $P$ and $Q$ are satisfied.

Putting together the above regions, we get Lemma 13.

Lemma 14. Assume that $P Q R L=Y, q \leq Q, \chi$ is a character $\bmod q$, $P(s, \chi), Q(s, \chi)$ and $R(s, \chi)$ are Dirichlet polynomials, and

$$
F(s, \chi)=P(s, \chi) Q(s, \chi) R(s, \chi) \sum_{l \sim L} \frac{\chi(l)}{l^{s}} .
$$

Let $\eta=q Q / Y, b=1+1 / \log N, T_{1}=\sqrt{L / q}$. Moreover assume that

$$
Y^{\frac{16}{135}} \ll R \ll Q
$$

and that $P$ and $Q$ lie in one of the following regions:

$$
\begin{array}{ll}
Y^{\frac{16}{135}} \ll P \ll Y^{\frac{89}{462}}, & Y^{\frac{16}{135}} \ll Q \ll P \\
Y^{\frac{89}{462}} \ll P \ll Y^{\frac{17}{54}}, & Y^{\frac{16}{135}} \ll Q \ll P^{-\frac{29}{48}} Y^{\frac{89}{288}} .
\end{array}
$$

Then for $T_{1} \leq T \leq Y$, we have

$$
\min ^{2}\left(\eta, \frac{1}{T}\right) \sum_{\chi(\bmod q)} \int_{T}^{2 T}|F(b+i t, \chi)|^{2} d t \ll \eta^{2} Y^{-\varepsilon} .
$$

Pr o of. Let $m=p q$ and $n=r$. An application of Lemma 6 yields that (10) holds under the following condition:

$$
M \ll Y^{\frac{3041}{7830}}, \quad N \ll M^{-1} Y^{\frac{19}{36}} .
$$

By the van der Corput method, it can be shown that for $T_{1} \leq|t| \leq Y$,

$$
\sum_{l \sim L} \frac{\chi(l)}{l^{b+i t}} \ll \sum_{a=1}^{q} \frac{1}{q}\left|\sum_{l_{1} \sim L / q} \frac{1}{\left(l_{1}+a / q\right)^{b+i t}}\right| \ll Y^{-\delta_{0}},
$$

where $\delta_{0}$ is a small positive constant. 
Using a similar discussion to that for Lemmas 7 and 8 with regions (i)-(vi) and (viii)-(xii), we can see that (10) holds under the conditions:

$$
Y^{\frac{17}{63}} \ll M \ll Y^{\frac{221}{693}}, \quad M^{-1} Y^{\frac{19}{36}} \ll N \ll M^{-\frac{1}{8}} Y^{\frac{7}{24}} ;
$$

$$
Y^{\frac{221}{693}} \ll M \ll Y^{\frac{109}{330}}, \quad M^{-1} Y^{\frac{19}{36}} \ll N \ll M^{-1} Y^{\frac{113}{198}} ;
$$

(d) $\quad Y^{\frac{109}{330}} \ll M \ll Y^{\frac{151}{450}}, \quad M^{-1} Y^{\frac{19}{36}} \ll N \ll M^{-6} Y^{\frac{20}{9}}$;

(e) $\quad Y^{\frac{151}{450}} \ll M \ll Y^{\frac{25}{72}}, \quad M^{-1} Y^{\frac{19}{36}} \ll N \ll M^{\frac{1}{4}} Y^{\frac{1}{8}}$;

(f) $\quad Y^{\frac{25}{72}} \ll M \ll Y^{\frac{10}{27}}, \quad M^{-1} Y^{\frac{19}{36}} \ll N \ll M^{-\frac{23}{15}} Y^{\frac{7}{9}}$;

(g) $\quad Y^{\frac{10}{27}} \ll M \ll Y^{\frac{617}{1620}}, \quad M^{-1} Y^{\frac{19}{36}} \ll N \ll M^{-1} Y^{\frac{47}{81}}$;

(h) $\quad Y^{\frac{617}{1620}} \ll M \ll Y^{\frac{3041}{7830}}, \quad M^{-1} Y^{\frac{19}{36}} \ll N \ll M^{-\frac{29}{19}} Y^{\frac{89}{114}}$;

(i) $\quad Y^{\frac{3041}{7830}} \ll M \ll Y^{\frac{3397}{7830}}, \quad Y^{\frac{16}{135}} \ll N \ll M^{-\frac{29}{19}} Y^{\frac{89}{114}}$.

In the regions:

$$
\begin{array}{ll}
Y^{\frac{16}{135}} \ll P \ll Y^{\frac{17}{126}}, & Y^{\frac{16}{135}} \ll Q \ll P ; \\
Y^{\frac{17}{126}} \ll P \ll Y^{\frac{143}{945}}, & Y^{\frac{16}{135}} \ll Q \ll P^{-1} Y^{\frac{17}{63}},
\end{array}
$$

condition (a) is satisfied.

In the regions:

$$
\begin{array}{ll}
Y^{\frac{17}{126}} \ll P \ll Y^{\frac{143}{945}}, & P^{-1} Y^{\frac{17}{63}} \ll Q \ll P ; \\
Y^{\frac{143}{945}} \ll P \ll Y^{\frac{221}{1386}}, & Y^{\frac{16}{135}} \ll Q \ll P ; \\
Y^{\frac{221}{1386}} \ll P \ll Y^{\frac{2083}{10395}}, & Y^{\frac{16}{135}} \ll Q \ll P^{-1} Y^{\frac{221}{693}},
\end{array}
$$

either condition (a) or (b) is satisfied.

In the regions:

$$
\begin{array}{ll}
Y^{\frac{221}{1386}} \ll P \ll Y^{\frac{109}{660}}, & P^{-1} Y^{\frac{221}{693}} \ll Q \ll P ; \\
Y^{\frac{109}{660}} \ll P \ll Y^{\frac{2083}{10395}}, & P^{-1} Y^{\frac{221}{693}} \ll Q \ll P^{-1} Y^{\frac{109}{330}} ; \\
Y^{\frac{2083}{10395}} \ll P \ll Y^{\frac{629}{2970}}, & Y^{\frac{16}{135}} \ll Q \ll P^{-1} Y^{\frac{109}{330}},
\end{array}
$$

either condition (a) or (c) is satisfied.

In the regions:

$$
\begin{array}{ll}
Y^{\frac{109}{660}} \ll P \ll Y^{\frac{151}{900}}, & P^{-1} Y^{\frac{109}{330}} \ll Q \ll P ; \\
Y^{\frac{151}{900}} \ll P \ll Y^{\frac{629}{2970}}, & P^{-1} Y^{\frac{109}{330}} \ll Q \ll P^{-1} Y^{\frac{151}{450}} ; \\
Y^{\frac{629}{2970}} \ll P \ll Y^{\frac{293}{1350}}, & Y^{\frac{16}{135}} \ll Q \ll P^{-1} Y^{\frac{151}{450}},
\end{array}
$$

either condition (a) or (d) is satisfied. 
In the regions:

$$
\begin{aligned}
& Y^{\frac{151}{900}} \ll P \ll Y^{\frac{25}{144}}, \quad P^{-1} Y^{\frac{151}{450}} \ll Q \ll P ; \\
& Y^{\frac{25}{144}} \ll P \ll Y^{\frac{293}{1350}}, \quad P^{-1} Y^{\frac{151}{450}} \ll Q \ll P^{-1} Y^{\frac{25}{72}} ; \\
& Y^{\frac{293}{1350}} \ll P \ll Y^{\frac{247}{1080}}, \quad Y^{\frac{16}{135}} \ll Q \ll P^{-1} Y^{\frac{25}{72}},
\end{aligned}
$$

either condition (a) or (e) is satisfied.

In the regions:

$$
\begin{array}{ll}
Y^{\frac{25}{144}} \ll P \ll Y^{\frac{5}{27}}, & P^{-1} Y^{\frac{25}{72}} \ll Q \ll P ; \\
Y^{\frac{5}{27}} \ll P \ll Y^{\frac{247}{1080}}, & P^{-1} Y^{\frac{25}{72}} \ll Q \ll P^{-1} Y^{\frac{10}{27}} ; \\
Y^{\frac{247}{1080}} \ll P \ll Y^{\frac{34}{135}}, & Y^{\frac{16}{135}} \ll Q \ll P^{-1} Y^{\frac{10}{27}},
\end{array}
$$

either condition (a) or (f) is satisfied.

In the regions:

$$
\begin{array}{ll}
Y^{\frac{5}{27}} \ll P \ll Y^{\frac{617}{3240}}, & P^{-1} Y^{\frac{10}{27}} \ll Q \ll P ; \\
Y^{\frac{617}{3240}} \ll P \ll Y^{\frac{34}{135}}, & P^{-1} Y^{\frac{10}{27}} \ll Q \ll P^{-1} Y^{\frac{617}{1620}} ; \\
Y^{\frac{34}{135}} \ll P \ll Y^{\frac{85}{324}}, & Y^{\frac{16}{135}} \ll Q \ll P^{-1} Y^{\frac{617}{1620}},
\end{array}
$$

either condition (a) or $(\mathrm{g})$ is satisfied.

In the regions:

$$
\begin{array}{ll}
Y^{\frac{617}{3240}} \ll P \ll Y^{\frac{89}{462}}, & P^{-1} Y^{\frac{617}{1620}} \ll Q \ll P ; \\
Y^{\frac{89}{462}} \ll P \ll Y^{\frac{85}{324}}, & P^{-1} Y^{\frac{617}{1620}} \ll Q \ll P^{-\frac{29}{48}} Y^{\frac{89}{288}} ; \\
Y^{\frac{85}{324}} \ll P \ll Y^{\frac{17}{54}}, & Y^{\frac{16}{135}} \ll Q \ll P^{-\frac{29}{48}} Y^{\frac{89}{288}},
\end{array}
$$

either condition (a) or (h) or (i) is satisfied.

Putting together the above regions, we get Lemma 14 .

\section{The remainder term in the sieve method}

Lemma 15. Suppose that $M H \ll Y^{1-6 \varepsilon} / Q, M H L=Y, a(m)=O(1)$, $b(h)=O(1), q \leq Q, \chi$ is a character $\bmod q, M(s, \chi)$ and $H(s, \chi)$ are Dirichlet polynomials, and

$$
F(s, \chi)=M(s, \chi) H(s, \chi) \sum_{l \sim L} \frac{\chi(l)}{l^{s}} .
$$

Let $\eta=q Q / Y, b=1+1 / \log N$ and $T_{1}=\sqrt{L / q}$. Assume further that for $T_{1} \leq T \leq Y$,

$$
\min ^{2}\left(\eta, \frac{1}{T}\right) \sum_{\chi(\bmod q)} \int_{T}^{2 T}|F(b+i t, \chi)|^{2} d t \ll \eta^{2} Y^{-\varepsilon} .
$$


Then for $N<n \leq N+A$, except for $O\left(A \log ^{-B} N\right)$ values, we have

$$
\sum_{\substack{m \sim M, h \sim H \\(m, n)=(h, n)=1}} a(m) b(h)\left(\sum_{\substack{m h l+p=n \\ N-Y<p \leq N \\ m h l \leq 2 \bar{Y}}} 1-\frac{1}{\varphi(m h)} \cdot \frac{Y}{\log N}\right)=O\left(Y \log ^{-B} N\right) .
$$

Proof. Set

$$
\begin{aligned}
\Sigma_{1} & =\sum_{\substack{m \sim M, h \sim H \\
(m, n)=(h, n)=1}} a(m) b(h) \sum_{\substack{m h l+p=n \\
N-Y<p \leq N \\
m h l \leq 2 Y}} 1 \\
& =\int_{\frac{1}{Q}}^{1+\frac{1}{Q}} \sum_{\substack{m h l \leq 2 Y \\
m \sim M, h \sim H \\
(m, n)=(h, n)=1}} a(m) b(h) e(\theta m h l) \sum_{N-Y<p \leq N} e(p \theta) e(-n \theta) d \theta
\end{aligned}
$$

and

$$
\begin{aligned}
S(\theta, n)= & \sum_{\substack{m h l \leq 2 Y \\
m \sim M, h \sim H \\
m, n)=(h, n)=1}} a(m) b(h) e(\theta m h l) \\
= & \sum_{\substack{m h l \leq 2 Y \\
m \sim M, h \sim H}} a(m) b(h) e(\theta m h l) \sum_{\substack{d_{1}\left|n \\
d_{1}\right| m}} \mu\left(d_{1}\right) \sum_{\substack{d_{2}\left|n \\
d_{2}\right| m}} \mu\left(d_{2}\right) \\
= & \sum_{d_{1}\left|n, d_{2}\right| n} \mu\left(d_{1}\right) \mu\left(d_{2}\right) \sum_{\substack{m h l \leq 2 Y \\
d_{1}\left|m, d_{2}\right| h}} a(m) b(h) e(\theta m h l) \\
= & \sum_{\substack{d_{1}\left|n, d_{2}\right| n \\
d_{1}, d_{2} \leq \log ^{12 B} N}}+\sum_{d_{1}\left|n, d_{2}\right| n}^{*}=S_{1}(\theta, n)+S_{2}(\theta, n),
\end{aligned}
$$

where "*" denotes that one of $d_{1}, d_{2}$ is larger than $\log ^{12 B} N$. Let

$$
S_{3}(\theta)=\sum_{N-Y<p \leq N} e(p \theta) .
$$

Then

$$
\begin{aligned}
\left|\int_{E_{2}} S_{2}(\theta, n) S_{3}(\theta) e(-n \theta) d \theta\right|^{2} & \\
& \leq \int_{0}^{1}\left|S_{2}(\theta, n)\right|^{2} d \theta \int_{0}^{1}\left|S_{3}(\theta)\right|^{2} d \theta
\end{aligned}
$$




$$
\begin{aligned}
& \ll d^{2}(n) Y \int_{0}^{1} \sum_{d_{1}\left|n, d_{2}\right| n}^{*}\left|\sum_{\substack{m h l \leq 2 Y \\
d_{1}\left|m, d_{2}\right| h}} a(m) b(h) e(\theta m h l)\right|^{2} d \theta \\
& \ll d^{2}(n) Y \sum_{d_{1}\left|n, d_{2}\right| n}^{*} \sum_{\substack{r \leq 2 Y \\
d_{1}\left|r, d_{2}\right| r}} d_{3}^{2}(r) .
\end{aligned}
$$

By Lemma 1, the last expression is

$$
\ll d_{3}^{6}(n) Y \log ^{8} N \sum_{d_{1}\left|n, d_{2}\right| n}^{*}\left(\frac{Y}{\left[d_{1}, d_{2}\right]}+Y^{\varepsilon}\right) \ll d_{3}^{8}(n) Y^{2} \log ^{-11 B} N .
$$

By Lemma 1, except for $O\left(A \log ^{-B} N\right)$ values, $d_{3}(n) \leq \log ^{B+2} N$. So,

$$
\int_{E_{2}} S_{2}(\theta, n) S_{3}(\theta) e(-n \theta) d \theta=O\left(Y \log ^{-B} N\right) .
$$

Next,

$$
\begin{aligned}
& \left|\int_{E_{2}} S_{1}(\theta, n) S_{3}(\theta) e(-n \theta) d \theta\right| \\
\leq & \sum_{d_{1} \leq \log ^{12 B} N} \sum_{d_{2} \leq \log ^{12 B} N}\left|\int_{E_{2}} \sum_{\begin{array}{c}
m h l \leq 2 Y \\
d_{1}\left|m, d_{2}\right| h
\end{array}} a(m) b(h) e(\theta m h l) S_{3}(\theta) e(-n \theta) d \theta\right| .
\end{aligned}
$$

Let

$$
S_{4}(\theta)=\sum_{\substack{m h l \leq 2 Y \\ m \sim M, h \sim H}} a(m) b(h) e(\theta m h l) .
$$

In the following we shall estimate

$$
\Sigma_{2}=\sum_{N<n \leq N+A}\left|\int_{E_{2}} S_{4}(\theta) S_{3}(\theta) e(-n \theta) d \theta\right|^{2} .
$$

We have

$$
\begin{aligned}
\Sigma_{2} & =\sum_{N<n \leq N+A} \int_{E_{2}} S_{4}(\xi) S_{3}(\xi) e(-n \xi) d \xi \int_{E_{2}} \overline{S_{4}(\alpha) S_{3}(\alpha)} e(n \alpha) d \alpha \\
& \ll \int_{E_{2}}\left|S_{4}(\xi) S_{3}(\xi)\right| d \xi\left(\int_{E_{2}}\left|S_{4}(\alpha) S_{3}(\alpha)\right| \min \left(A, \frac{1}{\|\alpha-\xi\|}\right) d \alpha\right) .
\end{aligned}
$$

Now,

$$
\int_{E_{2}}\left|S_{4}(\alpha) S_{3}(\alpha)\right| \min \left(A, \frac{1}{\|\alpha-\xi\|}\right) d \alpha
$$




$$
\begin{aligned}
& \leq\left(\int_{E_{2}}\left|S_{3}(\alpha)\right|^{2} d \alpha\right)^{\frac{1}{2}}\left(\int_{E_{2}}\left|S_{4}(\alpha)\right|^{2} \min ^{2}\left(A, \frac{1}{\|\alpha-\xi\|}\right) d \alpha\right)^{\frac{1}{2}} \\
& \ll Y^{\frac{1}{2}} \sup _{\xi \in[0,1]}\left(\int_{E_{2}}\left|S_{4}(\alpha)\right|^{2} \min ^{2}\left(A, \frac{1}{\|\alpha-\xi\|}\right) d \alpha\right)^{\frac{1}{2}} .
\end{aligned}
$$

Consequently,

$$
\Sigma_{2} \ll Y^{\frac{3}{2}} \log ^{4} N \sup _{\xi \in[0,1]}\left(\int_{E_{2}}\left|S_{4}(\alpha)\right|^{2} \min ^{2}\left(A, \frac{1}{\|\alpha-\xi\|}\right) d \alpha\right)^{\frac{1}{2}} .
$$

In order to prove

$$
\Sigma_{2} \ll A Y^{2} \log ^{-52 B} N
$$

we have to prove that

$$
\int_{D(\xi)}\left|S_{4}(\alpha)\right|^{2} d \alpha \ll Y \log ^{-120 B} N
$$

uniformly for $\xi \in[0,1]$, where

$$
D(\xi)=\left(\xi-\frac{\log ^{128 B} N}{A}, \xi+\frac{\log ^{128 B} N}{A}\right) \cap E_{2} .
$$

Let

$$
\begin{aligned}
J(a, q) & =\left(\frac{a}{q}-\frac{1}{q Q}, \frac{a}{q}+\frac{1}{q Q}\right), \\
\Omega(a, q) & = \begin{cases}J(a, q)-I(a, q) & \text { if } q \leq \log ^{E} N, \\
J(a, q) & \text { if } q>\log ^{E} N .\end{cases}
\end{aligned}
$$

Note that $1 /(q Q) \geq 1 / Q^{2}=\left(4 \log ^{128 B} N\right) / A$. By a simple argument (see Section 5 of [17]), $D(\xi)$ can be covered by at most two $\Omega(a, q)$. The proof of (17) reduces to showing that

$$
\max _{\substack{q \leq Q \\(a, q)=1}} \int_{\Omega(a, q)}\left|S_{4}(\alpha)\right|^{2} d \alpha \ll Y \log ^{-120 B} N .
$$

Let $\alpha=a / q+\beta$ with $\beta \in \Phi(q)$, where

$$
\Phi(q)= \begin{cases}\left\{\beta: \frac{\log ^{2 E} N}{q Y} \leq|\beta| \leq \frac{1}{q Q}\right\} & \text { if } q \leq \log ^{E} N, \\ \left\{\beta:|\beta| \leq \frac{1}{q Q}\right\} & \text { if } q>\log ^{E} N .\end{cases}
$$

Let $\Gamma=\max \left(q^{2 \varepsilon}, \log ^{260 B} N\right)$ and $\Gamma \geq q^{\varepsilon} \log ^{130 B} N$. 
Assume that $d_{1}=(m, q), d_{2}=(h, q), q_{1}=q / d_{1}, q_{2}=q / d_{2}, M_{1}=M / d_{1}$, $H_{1}=H / d_{2}, d^{\prime}=d_{1} d_{2} /\left(q, d_{1} d_{2}\right), q^{\prime}=q /\left(q, d_{1} d_{2}\right)$, and $d=\left(l, q^{\prime}\right)$. Then

(19) $\begin{aligned} S_{4}(\alpha) & =\sum_{\substack{m h l \leq 2 Y \\ d_{1}, d_{2}, d \leq \Gamma}} a(m) b(h) e(\alpha m h l)+\sum_{m h l \leq 2 Y}^{\prime} a(m) b(h) e(\alpha m h l) \\ & =S_{5}(\alpha)+S_{6}(\alpha)\end{aligned}$

where "'" denotes that one of $d_{1}, d_{2}, d$ is larger than $\Gamma$.

Let $\sum^{\prime \prime}$ be the part of $\sum^{\prime}$ with $d_{1}>\Gamma$. Then

$$
\begin{aligned}
\int_{\Omega(a, q)}\left|\sum_{m h l \leq 2 Y}^{\prime \prime} a(m) b(h) e(\alpha m h l)\right|^{2} d \alpha & \\
& \leq \int_{0}^{1}\left|\sum_{m h l \leq 2 Y}^{\prime \prime} a(m) b(h) e(\alpha m h l)\right|^{2} d \alpha \\
& \ll \sum_{k \mid q} \sum_{\substack{r \leq 2 Y \\
k>\Gamma}} d_{3}^{2}(r) \ll Y \log ^{8} N \sum_{k \mid q} \frac{d_{3}^{2}(k)}{k} \\
& \ll Y \log ^{8} N \cdot \frac{q^{\varepsilon}}{\Gamma} \ll Y \log ^{-120 B} N .
\end{aligned}
$$

Hence,

$$
\int_{\Omega(a, q)}\left|S_{6}(\alpha)\right|^{2} d \alpha \ll Y \log ^{-120 B} N .
$$

Next,

$$
\begin{aligned}
S_{5}(\alpha)= & \sum_{\substack{d_{1} \mid q \\
d_{1} \leq \Gamma}} \sum_{\substack{d_{2} \mid q \\
d_{2} \leq \Gamma}} \sum_{\substack{m_{1} \sim M_{1} \\
\left(m_{1}, q_{1}\right)=1}} a\left(m_{1} d_{1}\right) \sum_{\substack{h_{1} \sim H_{1} \\
\left(h_{1}, q_{2}\right)=1}} b\left(h_{1} d_{2}\right) \\
& \times \sum_{\substack{m_{1} \\
h_{1} l \leq 2 Y /\left(d_{1} d_{2}\right) \\
\left(l, q^{\prime}\right) \leq \Gamma}} e\left(\frac{a d^{\prime} m_{1} h_{1} l}{q^{\prime}}\right) e\left(\beta d_{1} d_{2} m_{1} h_{1} l\right) \\
= & \sum_{\substack{d_{1} \mid q \\
d_{1} \leq \Gamma}} \sum_{\substack{d_{2} \mid q \\
d_{2} \leq \Gamma}} \sum_{\substack{d \mid q^{\prime} \\
d \leq \Gamma}} \sum_{\substack{m_{1} \sim M_{1} \\
\left(m_{1}, q_{1}\right)=1}} a\left(m_{1} d_{1}\right) \sum_{\substack{h_{1} \sim H_{1} \\
\left(h_{1}, q_{2}\right)=1}} b\left(h_{1} d_{2}\right) \\
& \times \sum_{\substack{m_{1} h_{1} l_{1} \leq 2 Y /\left(d d_{1} d_{2}\right) \\
\left(l_{1}, q^{\prime \prime}\right)=1}} e\left(\frac{a d^{\prime} m_{1} h_{1} l_{1}}{q^{\prime \prime}}\right) e\left(\beta d d_{1} d_{2} m_{1} h_{1} l_{1}\right),
\end{aligned}
$$

where $q^{\prime \prime}=q^{\prime} / d$ and $l_{1}=l / d$. We have $q^{\prime} \mid q_{1}$ and $\left(m_{1}, q_{1}\right)=1$, so $\left(m_{1}, q^{\prime}\right)=1$; 
$q^{\prime} \mid q_{2}$ and $\left(h_{1}, q_{2}\right)=1$, so $\left(h_{1}, q^{\prime}\right)=1 ;$ and $\left(a d^{\prime}, q^{\prime}\right)=1$. Therefore

$$
\begin{aligned}
& \int_{\Omega(a, q)}\left|S_{5}(\alpha)\right|^{2} d \alpha \\
& \ll q^{\varepsilon} \sum_{\substack{d_{1} \mid q \\
d_{1} \leq \Gamma}} \sum_{\substack{d_{2} \mid q \\
d_{2} \leq \Gamma}} \sum_{\substack{d \mid q^{\prime} \\
d \leq \Gamma}} \int_{\Phi(q)} \mid \sum_{\substack{m_{1} \sim M_{1} \\
\left(m_{1}, q_{1}\right)=1}} a\left(m_{1} d_{1}\right) \sum_{\substack{h_{1} \sim H_{1} \\
\left(h_{1}, q_{2}\right)=1}} b\left(h_{1} d_{2}\right) \\
& \times\left.\sum_{\substack{m_{1} h_{1} l_{1} \leq 2 Y /\left(d d_{1} d_{2}\right) \\
\left(l_{1}, q^{\prime \prime}\right)=1}} e\left(\frac{a d^{\prime} m_{1} h_{1} l_{1}}{q^{\prime \prime}}\right) e\left(\beta d d_{1} d_{2} m_{1} h_{1} l_{1}\right)\right|^{2} d \beta .
\end{aligned}
$$

In the following we first assume that $d_{1}=d_{2}=d=1$ and set

$$
S_{7}(\beta)=\sum_{\substack{m h l \leq 2 Y \\ m \sim M, h \sim H \\(m h l, q)=1}} a(m) b(h) e\left(\frac{a m h l}{q}\right) e(\beta m h l) .
$$

We proceed to prove

$$
\int_{\Phi(q)}\left|S_{7}(\beta)\right|^{2} d \beta \ll \frac{Y}{q \log ^{E} N}+Y^{1-\frac{\varepsilon}{2}} .
$$

We have

$$
\begin{aligned}
S_{7}(\beta)= & \frac{1}{\varphi(q)} \sum_{\chi(\bmod q)} \chi(a) \tau(\bar{\chi}) \sum_{\substack{m h l \leq 2 Y \\
m \sim M, h \sim H}} a(m) \chi(m) b(h) \chi(h) \chi(l) e(\beta m h l) \\
= & \frac{1}{\varphi(q)} \sum_{\chi(\bmod q)} \chi(a) \tau(\bar{\chi}) W(\chi, \beta) \\
& +\frac{\mu(q)}{q} \sum_{\substack{m \sim M \\
(m, q)=1}} \frac{a(m)}{m} \sum_{\substack{h \sim H \\
(h, q)=1}} \frac{b(h)}{h} \sum_{r \leq 2 Y} e(\beta r) \\
= & S_{8}(\beta)+S_{9}(\beta),
\end{aligned}
$$

where

$$
\begin{aligned}
W(\chi, \beta)= & \sum_{\substack{m h l \leq 2 Y \\
m \sim M, h \sim H}} a(m) \chi(m) b(h) \chi(h) \chi(l) e(\beta m h l) \\
& -E_{0} \frac{\varphi(q)}{q} \sum_{\substack{m \sim M \\
(m, q)=1}} \frac{a(m)}{m} \sum_{\substack{h \sim H \\
(h, q)=1}} \frac{b(h)}{h} \sum_{r \leq 2 Y} e(\beta r) .
\end{aligned}
$$


When $q \leq \log ^{E} N$,

$$
\begin{aligned}
\int_{\Phi(q)}\left|S_{9}(\beta)\right|^{2} d \beta & \ll \frac{1}{q^{2}} \int_{\Phi(q)}\left|\sum_{r \leq 2 Y} e(\beta r)\right|^{2} d \beta \\
& \ll \frac{1}{q^{2}} \int_{\log ^{2 E}}^{\infty} \frac{d \beta}{\int_{N /(q Y)}} \ll \frac{Y}{q \log ^{E} N} .
\end{aligned}
$$

When $q>\log ^{E} N$,

$$
\int_{\Phi(q)}\left|S_{9}(\beta)\right|^{2} d \beta \ll \frac{1}{q^{2}} \int_{0}^{1}\left|\sum_{r \leq 2 Y} e(\beta r)\right|^{2} d \beta \ll \frac{Y}{q^{2}} \ll \frac{Y}{q \log ^{E} N} .
$$

So, we always have

$$
\int_{\Phi(q)}\left|S_{9}(\beta)\right|^{2} d \beta \ll \frac{Y}{q \log ^{E} N} .
$$

By Lemma 2,

$$
\int_{\Phi(q)}\left|S_{8}(\beta)\right|^{2} d \beta \ll \log N \sum_{\chi(\bmod q)} \int_{-\frac{1}{q Q}}^{\frac{1}{q Q}}|W(\chi, \beta)|^{2} d \beta
$$

and

$$
W(\chi, \beta)=\sum_{r \leq 2 Y}\left(\lambda(r) \chi(r)-E_{0} I\right) e(\beta r),
$$

where

$$
\lambda(r)=\sum_{\substack{m h l=r \\ m \sim M, h \sim H}} a(m) b(h), \quad I=\frac{\varphi(q)}{q} \sum_{\substack{m \sim M \\(m, q)=1}} \frac{a(m)}{m} \sum_{\substack{h \sim H \\(h, q)=1}} \frac{b(h)}{h} .
$$

By Lemma 3,

$$
\begin{aligned}
& \int_{-\frac{1}{q Q}}^{\frac{1}{q Q}}|W(\chi, \beta)|^{2} d \beta \\
& \ll \frac{1}{(q Q)^{2}} \int_{-\infty}^{\infty}\left|\sum_{\substack{x<r \leq x+q Q / 2 \\
1 \leq r \leq 2 Y}}\left(\lambda(r) \chi(r)-E_{0} I\right)\right|^{2} d x \\
& =\frac{1}{(q Q)^{2}} \int_{(q Q)^{2}}^{2 Y}\left|\sum_{x<r \leq x+q Q / 2}\left(\lambda(r) \chi(r)-E_{0} I\right)\right|^{2} d x+O\left(Q^{4} \log ^{4} N\right) .
\end{aligned}
$$

The contribution of the term $O\left(Q^{4} \log ^{4} N\right)$ to $(24)$ is $O\left(Y^{1-\varepsilon}\right)$. 
By Theorem 2 on p. 34 of [15],

$$
\sum_{\chi(\bmod q)}\left|\sum_{x<r \leq x+q Q / 2} \lambda(r) \chi(r)\right|^{2} \ll q Q \sum_{x<r \leq x+q Q / 2}|\lambda(r)|^{2} \ll(q Q)^{2} \log ^{8} N
$$

and

Hence,

$$
\sum_{\chi(\bmod q)}\left|\sum_{x<r \leq x+q Q / 2} E_{0} I\right|^{2} \ll(q Q)^{2}
$$

$$
\frac{1}{(q Q)^{2}} \int_{(q Q)^{2}}^{Y^{1-2 \varepsilon}} \sum_{\chi(\bmod q)}\left|\sum_{x<r \leq x+q Q / 2}\left(\lambda(r) \chi(r)-E_{0} I\right)\right|^{2} d x \ll Y^{1-\varepsilon} .
$$

By the discussion in Lemma 6 of [19] (see also (24) of [17]), we have

$$
\begin{aligned}
\frac{1}{(q Q)^{2}} \int_{Y^{1-2 \varepsilon}}^{2 Y} & \sum_{\chi(\bmod q)}\left|\sum_{x<r \leq x+q Q / 2}\left(\lambda(r) \chi(r)-E_{0} I\right)\right|^{2} d x \\
\ll \frac{\log N}{(q Q)^{2}} \sup _{Y^{1-2 \varepsilon} \leq V \leq 2 Y} \sup _{q Q /(20 V) \leq \eta \leq 20 q Q / V} & \\
& \int_{V}^{3 V} \sum_{\chi(\bmod q)}\left|\sum_{x<r \leq x+\eta x}\left(\lambda(r) \chi(r)-E_{0} I\right)\right|^{2} d x .
\end{aligned}
$$

We only estimate for $V=Y$ and $\eta=q Q / Y$ the quantity

$$
\Sigma_{3}=\frac{1}{(q Q)^{2}} \sum_{\chi(\bmod q)} \int_{Y}^{3 Y}\left|\sum_{x<r \leq x+\eta x}\left(\lambda(r) \chi(r)-E_{0} I\right)\right|^{2} d x .
$$

The others can be dealt with in the same way.

Assume $M H \leq Q Y^{-\varepsilon} / \sqrt{q}$. The trivial estimation yields

$$
\begin{aligned}
\sum_{x<r \leq x+\eta x} \lambda(r) \chi(r) & \\
& =\sum_{m \sim M} a(m) \chi(m) \sum_{h \sim H} b(h) \chi(h) \sum_{x /(m h)<l \leq(x+\eta x) /(m h)} \chi(l) \\
& =\sum_{m \sim M} a(m) \chi(m) \sum_{h \sim H} b(h) \chi(h)\left(E_{0} \frac{\varphi(q)}{q} \cdot \frac{\eta x}{m h}+O(q)\right) \\
& =\eta x E_{0} I+O(q M H) .
\end{aligned}
$$

Hence,

$$
\Sigma_{3} \ll \frac{1}{(q Q)^{2}} q Y(q M H)^{2} \ll Y^{1-\varepsilon} .
$$


We can assume

$$
M H>\frac{Q Y^{-\varepsilon}}{\sqrt{q}} .
$$

Let $b=1+1 / \log N, M H L=Y$. Perron's formula yields

$$
\sum_{x<r \leq x+\eta x} \lambda(r) \chi(r)=\frac{1}{2 \pi i} \int_{b-i Y}^{b+i Y} F(s, \chi) \frac{(1+\eta)^{s}-1}{s} x^{s} d s+O\left(Y^{\varepsilon}\right) .
$$

If $s=b+i t$ and $|t| \leq c_{1} L / q$, by Theorem 1 on p. 447 of [16], we have

$$
\sum_{c_{1} L<l \leq c_{2} L} \frac{\chi(l)}{l^{s}}=E_{0} \frac{\varphi(q)}{q} \cdot \frac{\left(c_{2} L\right)^{1-s}-\left(c_{1} L\right)^{1-s}}{1-s}+O\left(\frac{q}{L}\right) .
$$

Moreover,

$$
\frac{(1+\eta)^{s}-1}{s} x^{s}=\eta x^{s}+O\left(|s| \eta^{2} x\right), \quad \frac{(1+\eta)^{s}-1}{s} x^{s} \ll \eta x .
$$

Let $T_{1}=\sqrt{L / q}$. Then

$$
\begin{aligned}
& \frac{1}{2 \pi i} \int_{b-i T_{1}}^{b+i T_{1}} F(s, \chi) \frac{(1+\eta)^{s}-1}{s} x^{s} d s \\
& =\eta E_{0} \cdot \frac{\varphi(q)}{q} \cdot \frac{1}{2 \pi i} \int_{b-i T_{1}}^{b+i T_{1}} M(s, \chi) H(s, \chi) \frac{\left(c_{2} L\right)^{1-s}-\left(c_{1} L\right)^{1-s}}{1-s} x^{s} d s \\
& \quad+O\left(S_{1}\right)+O\left(S_{2}\right),
\end{aligned}
$$

where

$$
\begin{aligned}
& S_{1}=\frac{q}{L} \eta Y \int_{-T_{1}}^{T_{1}}|M(b+i t, \chi) H(b+i t, \chi)| d t, \\
& S_{2}=\eta^{2} Y \int_{-T_{1}}^{T_{1}}|M(b+i t, \chi) H(b+i t, \chi)| d t .
\end{aligned}
$$

By Lemma 4 , the contribution of $S_{1}$ to $(25)$ is

$$
\begin{aligned}
& \ll \frac{1}{(q Q)^{2}} Y\left(\frac{q}{L} \cdot q Q\right)^{2} \sum_{\chi(\bmod q)} T_{1} \int_{-T_{1}}^{T_{1}}|M(b+i t, \chi) H(b+i t, \chi)|^{2} d t \\
& \ll Y T_{1}\left(\frac{q}{L}\right)^{2}\left(1+\frac{q T_{1}}{M H}\right) \log ^{3} N \ll Y^{1-\varepsilon} .
\end{aligned}
$$


The contribution of $S_{2}$ to $(25)$ is

$$
\begin{aligned}
& \ll \frac{1}{(q Q)^{2}} Y\left(\frac{q Q}{Y} \cdot q Q\right)^{2} \sum_{\chi(\bmod q)} T_{1} \int_{-T_{1}}^{T_{1}}|M(b+i t, \chi) H(b+i t, \chi)|^{2} d t \\
& \ll Y T_{1}\left(\frac{q Q}{Y}\right)^{2}\left(1+\frac{q T_{1}}{M H}\right) \log ^{3} N \ll Y^{1-\varepsilon} .
\end{aligned}
$$

By Perron's formula again,

$$
\begin{aligned}
\eta E_{0} \cdot \frac{\varphi(q)}{q} \cdot \frac{1}{2 \pi i} \int_{b-i T_{1}}^{b+i T_{1}} M(s, \chi) H(s, \chi) \frac{\left(c_{2} L\right)^{1-s}-\left(c_{1} L\right)^{1-s}}{1-s} x^{s} d s \\
=\eta x E_{0} \frac{\varphi(q)}{q} \sum_{m \sim M} \frac{a(m) \chi(m)}{m} \sum_{h \sim H} \frac{b(h) \chi(h)}{h} \\
\quad+O\left(\frac{\eta x E_{0} Y^{\varepsilon}}{T_{1}}\right)+O\left(\frac{\eta x E_{0} Y^{\varepsilon}}{M H}\right) \\
=\eta x E_{0} I+O\left(\frac{\eta x E_{0} Y^{\varepsilon}}{T_{1}}\right)+O\left(\frac{\eta x E_{0} Y^{\varepsilon}}{M H}\right) .
\end{aligned}
$$

The contribution of the term $O\left(\eta x E_{0} Y^{\varepsilon} / T_{1}\right)$ to $(25)$ is

$$
\ll \frac{1}{(q Q)^{2}} Y\left(\frac{q Q Y^{\varepsilon}}{T_{1}}\right)^{2} \ll Y^{1-\varepsilon} .
$$

The contribution of the term $O\left(\eta x E_{0} Y^{\varepsilon} /(M H)\right)$ to $(25)$ is

$$
\ll \frac{1}{(q Q)^{2}} Y\left(\frac{q Q Y^{\varepsilon}}{M H}\right)^{2} \ll Y^{1-\varepsilon} .
$$

Combining the above, we have

$$
\begin{aligned}
& \text { (28) } \Sigma_{3} \ll \frac{\log ^{2} N}{(q Q)^{2}} \\
& \times \max _{T_{1} \leq|T| \leq Y} \sum_{\chi(\bmod q)} \int_{Y}^{3 Y}\left|\int_{b+i T}^{b+2 i T} F(s, \chi) \frac{(1+\eta)^{s}-1}{s} x^{s} d s\right|^{2} d x+O\left(Y^{1-\varepsilon}\right) .
\end{aligned}
$$

Let

$$
\varrho(s)=\frac{(1+\eta)^{s}-1}{s} .
$$

If $s=b+i t$ and $|\operatorname{Im}(s)| \sim T$, then $\varrho(s) \ll \min (\eta, 1 / T)$. Thus 


$$
\begin{aligned}
& \int_{Y}^{3 Y}\left|\int_{b+i T}^{b+2 i T} F(s, \chi) \varrho(s) x^{s} d s\right|^{2} d x \\
& =\int_{Y}^{3 Y} d x \int_{b+i T}^{b+2 i T} d s_{1} \int_{b+i T}^{b+2 i T} F\left(s_{1}, \chi\right) \overline{F\left(s_{2}, \chi\right)} \varrho\left(s_{1}\right) \overline{\varrho\left(s_{2}\right)} x^{s_{1}+\bar{s}_{2}} \overline{d s_{2}} \\
& \quad \ll \min ^{2}\left(\eta, \frac{1}{T}\right) \int_{b+i T}^{b+2 i T}\left|d s_{1}\right| \int_{b+i T}^{b+2 i T}\left|F\left(s_{1}, \chi\right) F\left(s_{2}, \chi\right)\right|\left|\int_{Y}^{3 Y} x^{s_{1}+\bar{s}_{2}} d x\right|\left|d s_{2}\right| \\
& \quad \ll \min ^{2}\left(\eta, \frac{1}{T}\right) Y^{3} \int_{b+i T}^{b+2 i T}\left|d s_{1}\right| \int_{b+i T}^{b+2 i T} \frac{\left|F\left(s_{1}, \chi\right)\right|^{2}+\left|F\left(s_{2}, \chi\right)\right|^{2}}{\left|1+s_{1}+\bar{s}_{2}\right|}\left|d s_{2}\right| \\
& \quad \ll \min ^{2}\left(\eta, \frac{1}{T}\right) Y^{3} \log N \int_{T}^{2 T}|F(b+i t, \chi)|^{2} d t .
\end{aligned}
$$

Consequently

$$
\text { (29) } \begin{aligned}
\Sigma_{3} \ll & \frac{Y^{3} \log ^{3} N}{(q Q)^{2}} \\
& \times \max _{T_{1} \leq T \leq Y} \min ^{2}\left(\eta, \frac{1}{T}\right) \sum_{\chi(\bmod q)} \int_{T}^{2 T}|F(b+i t, \chi)|^{2} d t+O\left(Y^{1-\varepsilon}\right) .
\end{aligned}
$$

By the assumption in Lemma $15, \Sigma_{3} \ll Y^{1-\frac{\varepsilon}{2}}$. Hence, (22) holds.

In the same way, we can prove

$$
\begin{array}{r}
\int_{\Phi(q)} \mid \sum_{\substack{m_{1} \sim M_{1} \\
\left(m_{1}, q_{1}\right)=1}} a\left(m_{1} d_{1}\right) \sum_{\substack{h_{1} \sim H_{1} \\
\left(h_{1}, q_{2}\right)=1}} b\left(h_{1} d_{2}\right) \sum_{\substack{m_{1} h_{1} l_{1} \leq 2 Y /\left(d d_{1} d_{2}\right) \\
\left(l_{1}, q^{\prime \prime}\right)=1}} e\left(\frac{a d^{\prime} m_{1} h_{1} l_{1}}{q^{\prime \prime}}\right) \\
\times\left. e\left(\beta d d_{1} d_{2} m_{1} h_{1} l_{1}\right)\right|^{2} d \beta \ll \frac{q^{6 \varepsilon} Y}{q^{\prime \prime} \log ^{\frac{E}{2}} N}+Y^{1-\frac{\varepsilon}{2}} .
\end{array}
$$

From $q^{\prime \prime} \geq q^{1-6 \varepsilon} \log ^{-780 B} N$ and (21), it follows that

$$
\int_{\Omega(a, q)}\left|S_{5}(\alpha)\right|^{2} d \alpha \ll Y \log ^{-120 B} N .
$$

Hence, (18) holds. We have

$$
\Sigma_{2} \ll A Y^{2} \log ^{-52 B} N .
$$

In the same way, it can be proved that

$$
\sum_{N<n \leq N+A}\left|\int_{E_{2}} S_{1}(\theta, n) S_{3}(\theta) e(-n \theta) d \theta\right|^{2} \ll A Y^{2} \log ^{-4 B} N .
$$


So, except for $O\left(A \log ^{-B} N\right)$ values, we always have

$$
\int_{E_{2}} S_{1}(\theta, n) S_{3}(\theta) e(-n \theta) d \theta=O\left(Y \log ^{-B} N\right) .
$$

If $\theta=a / q+\beta \in E_{1}$, then

$$
S(\theta, n)=\sum_{\substack{k l \leq 2 Y \\ k \sim K,(k, n)=1}} g(k) e(\theta k l),
$$

where $K=M H$ and

$$
g(k)=\sum_{\substack{m h=k \\ m \sim M, h \sim H}} a(m) b(h) .
$$

If $q \nmid b$, then

$$
\sum_{l \leq x} e\left(\frac{b l}{q}\right)=O(q)
$$

From this and Abel's summation, it follows that

$$
\begin{aligned}
\sum_{\substack{k l \leq 2 Y \\
k \sim K,(k, n)=1 \\
q \nmid k}} g(k) e(\theta k l) & =\sum_{\substack{k \sim K \\
(k, n)=1, q \nmid k}} g(k) \sum_{l \leq 2 Y / k} e\left(\frac{a k l}{q}+\beta k l\right) \\
& \ll \sum_{\substack{k \sim K \\
k l \leq 2 Y \\
k \sim K,(k, n)=1 \\
q \mid k}} d(k) \log ^{2 E} N \ll Y^{1-2 \varepsilon}, \\
\sum_{l \leq 2 Y / k} e(\beta k l) & =\int_{\substack{k \sim K \\
(k, n)=1, q \mid k}}^{2 Y / k}\left(k(\beta) \sum_{l \leq 2 Y / k} e(\beta k l),\right. \\
& =\int_{1}^{2 Y / k} e(\beta k t) d t-\int_{1}^{2 Y / k} e(\beta k t) d(\{t\}) \\
& =\frac{1}{k} \int_{k}^{2 Y} e(\beta u) d u+O\left(\log ^{2 E} N\right) \\
& =\frac{1}{k} \sum_{r \leq 2 Y} e(\beta r)+O\left(\log ^{2 E} N\right),
\end{aligned}
$$


hence

$$
S(\theta, n)=\sum_{\substack{k \sim K \\(k, n)=1, q \mid k}} \frac{g(k)}{k} \sum_{r \leq 2 Y} e(\beta r)+O\left(Y^{1-2 \varepsilon}\right) .
$$

By the same discussion as in Lemma 10 of [7], we have

$$
\sum_{N-Y<p \leq N} e(p \theta)=\frac{\mu(q)}{\varphi(q) \log N} \sum_{N-Y<s \leq N} e(\beta s)+O(Y \exp (-c \sqrt{\log N})) .
$$

Set

$$
\begin{aligned}
\Sigma_{4}= & \int_{E_{1}} S(\theta, n) S_{3}(\theta) e(-n \theta) d \theta \\
= & \sum_{q \leq \log ^{E}} \sum_{\substack{a=1 \\
(a, q)=1}}^{q} \int_{\log ^{2 E}}^{\log ^{2 E} N_{N /(q Y)} /(q Y)} S\left(\frac{a}{q}+\beta, n\right) S_{3}\left(\frac{a}{q}+\beta\right) \\
& \times e\left(-\left(\frac{a}{q}+\beta\right) n\right) d \beta \\
= & \sum_{q \leq \log ^{E} N} \sum_{\substack{a=1 \\
(a, q)=1}}^{q} e\left(-\frac{a n}{q}\right)_{-\log ^{2 E}{ }_{N /(q Y)}}^{\log ^{2 E} N /(q Y)}\left\{\left(\sum_{\substack{k \sim K \\
(k, n)=1, q \mid k}} \frac{g(k)}{k}\right) \sum_{r \leq 2 Y} e(\beta r)\right. \\
& \left.\times \frac{\mu(q)}{\varphi(q) \log N} \sum_{N-Y<s \leq N} e(\beta s) e(-n \beta)\right\} d \beta+O\left(\frac{Y}{\log ^{B} N}\right) .
\end{aligned}
$$

Since

$$
\int_{-\log ^{2 E}}^{\log ^{2 E}} \sum_{N /(q Y)}^{N /(q Y)} e(\beta r) \sum_{N-Y<s \leq N} e(\beta s) e(-n \beta) d \beta=Y+O\left(\frac{q Y}{\log ^{2 E} N}\right) \text {, }
$$

we obtain

$$
\begin{aligned}
\Sigma_{4} & =\frac{Y}{\log N} \sum_{\substack{q \leq \log ^{E} N \\
(q, n)=1}} \frac{\mu(q)}{\varphi(q)} \sum_{\substack{a=1 \\
(a, q)=1}}^{q} e\left(-\frac{a n}{q}\right) \sum_{\substack{k \sim K \\
(k, n)=1, q \mid k}} \frac{g(k)}{k}+O\left(\frac{Y}{\log ^{B} N}\right) \\
& =\frac{Y}{\log N} \sum_{q \leq \log ^{E} N} \frac{\mu^{2}(q)}{\varphi(q)} \sum_{\substack{k \sim K \\
(k, n)=1, q \mid k}} \frac{g(k)}{k}+O\left(\frac{Y}{\log ^{B} N}\right) .
\end{aligned}
$$

Set

$$
\Omega=\sum_{q \leq \log ^{E} N} \frac{\mu^{2}(q)}{\varphi(q)} \sum_{\substack{k \sim K \\(k, n)=1, q \mid k}} \frac{g(k)}{k}=\sum_{\substack{k \sim K \\ k, n)=1}} \frac{g(k)}{k} \sum_{\substack{q \leq \log ^{E} N \\ q \mid k}} \frac{\mu^{2}(q)}{\varphi(q)} .
$$


We have

$$
\sum_{\substack{k \sim K \\(k, n)=1}} \frac{g(k)}{k} \sum_{\substack{q>\log ^{E} N \\ q \mid k}} \frac{\mu^{2}(q)}{\varphi(q)} \ll \frac{1}{\log ^{E-1} N} \sum_{k \sim K} \frac{d^{2}(k)}{k} \ll \frac{1}{\log ^{E-4} N} .
$$

Consequently,

$$
\begin{aligned}
\Omega & =\sum_{\substack{k \sim K \\
k, n)=1}} \frac{g(k)}{k} \sum_{q \mid k} \frac{\mu^{2}(q)}{\varphi(q)}+O\left(\frac{1}{\log ^{B} N}\right) \\
& =\sum_{\substack{k \sim K \\
k, n)=1}} \frac{g(k)}{\varphi(k)}+O\left(\frac{1}{\log ^{B} N}\right) \\
& =\sum_{\substack{m \sim M, h \sim H \\
(m, n)=(h, n)=1}} \frac{a(m) b(h)}{\varphi(m h)}+O\left(\frac{1}{\log ^{B} N}\right) .
\end{aligned}
$$

Thus

$$
\Sigma_{4}=\frac{Y}{\log N} \sum_{\substack{m \sim M, h \sim H \\(m, n)=(h, n)=1}} \frac{a(m) b(h)}{\varphi(m h)}+O\left(\frac{Y}{\log ^{B} N}\right),
$$

and so

$$
\Sigma_{1}=\frac{Y}{\log N} \sum_{\substack{m \sim M, h \sim H \\(m, n)=(h, n)=1}} \frac{a(m) b(h)}{\varphi(m h)}+O\left(\frac{Y}{\log ^{B} N}\right) .
$$

The proof of Lemma 15 is complete.

Lemma 16. Suppose that $a(p)=O(1), b(q)=O(1), c(r)=O(1)$,

$$
Y^{\frac{16}{135}} \ll R \ll Q,
$$

and that $P$ and $Q$ lie in one of the following regions:

$$
Y^{\frac{16}{135}} \ll P \ll Y^{\frac{89}{462}}, \quad Y^{\frac{16}{135}} \ll Q \ll P
$$

$$
Y^{\frac{89}{462}} \ll P \ll Y^{\frac{17}{54}}, \quad Y^{\frac{16}{135}} \ll Q \ll P^{-\frac{29}{48}} Y^{\frac{89}{288}} .
$$

Then for $N<n \leq N+A$, except for $O\left(A \log ^{-B} N\right)$ values, we have

$$
\begin{aligned}
\sum_{\substack{p \sim P \\
(p, n)=1}} \sum_{\substack{q \sim Q \\
(q, n)=1}} \sum_{\substack{r \sim R \\
(r, n)=1}} a(p) b(q) c(r)\left(\sum_{\substack{p q r l+p_{1}=n \\
N-Y<p_{1} \leq N \\
p q r l \leq 2 Y}} 1-\frac{1}{\varphi(p q r)} \cdot \frac{Y}{\log N}\right) \\
=O\left(Y \log ^{-B} N\right) .
\end{aligned}
$$


Proof. This follows from Lemmas 14 and 15.

Lemma 17. Suppose that $M \ll Y^{\frac{19}{36}}$ and $a(m)=O(1)$. Then for $N<$ $n \leq N+A$, except for $O\left(A \log ^{-B} N\right)$ values, we have

$$
\sum_{\substack{m \sim M \\(m, n)=1}} a(m)\left(\sum_{\substack{m l+p=n \\ N-Y<p \leq N \\ m l \leq 2 Y}} 1-\frac{1}{\varphi(m)} \cdot \frac{Y}{\log N}\right)=O\left(Y \log ^{-B} N\right) .
$$

Pr o of. This follows from Lemmas 6 and 15.

\section{Asymptotic formula}

Lemma 18. Suppose that $Q Y^{\varepsilon} \ll H K \ll Y^{1-\delta}, M H K=Y, 0 \leq b(h)=$ $O(1)$, and $0 \leq g(k)=O(1)$. If $h$ has a prime factor $<Y^{\delta}$, then $\bar{b}(h)=0$, and similarly for $g(k)$. Assume that $q \leq Q, \chi$ is a character $\bmod q, H(s, \chi)$ and $K(s, \chi)$ are Dirichlet polynomials,

$$
M(s, \chi)=\sum_{m \sim M} \frac{\Lambda(m) \chi(m)}{m^{s}}
$$

and $G(s, \chi)=M(s, \chi) H(s, \chi) K(s, \chi)$. Let $\eta=q Q / Y, b=1+1 / \log N$ and $T_{0}=\log \frac{E}{\delta^{2}} Y$. Assume further that for $T_{0} \leq T \leq Y$,

$$
\min ^{2}\left(\eta, \frac{1}{T}\right) \sum_{\chi(\text { good })} \int_{T}^{2 T}|G(b+i t, \chi)|^{2} d t \ll \eta^{2} \log ^{-6 E} N .
$$

Then for $N<n \leq N+A$, except for $O\left(A \log ^{-B} N\right)$ values, we have

$$
\begin{aligned}
\sum_{\substack{h k p+p_{1}=n \\
N-Y<p_{1} \leq N \\
h k p \leq 2 Y \\
h \sim H, k \sim K}} b(h) g(k) & =\frac{C(n)}{\log N}\left(1+O\left(\frac{1}{\log N}\right)\right) \\
& \times \sum_{h \sim H, k \sim K} b(h) g(k) \sum_{Y /(h k)<p \leq 2 Y /(h k)} 1+O\left(Y \log ^{-B} N\right),
\end{aligned}
$$

where

$$
C(n)=\prod_{p \nmid n}\left(1-\frac{1}{(p-1)^{2}}\right) \prod_{p \mid n}\left(1+\frac{1}{p-1}\right) .
$$


Proof. Set

$$
\begin{aligned}
\Sigma_{1} & =\sum_{\substack{h \sim H \\
k \sim K}} b(h) g(k) \sum_{\substack{h k p+p_{1}=n \\
N-Y<p_{1} \leq N \\
h k p \leq 2 Y}} 1 \\
& =\int_{\frac{1}{Q}}^{1+\frac{1}{Q}} \sum_{\substack{h k p \leq 2 Y \\
h \sim H, k \sim K}} b(h) g(k) e(\theta h k p) \sum_{N-Y<p_{1} \leq N} e\left(p_{1} \theta\right) e(-n \theta) d \theta .
\end{aligned}
$$

In the following we often use the definitions and the argument of Lemma 15. Write

$$
S_{1}(\theta)=\sum_{\substack{h k p \leq 2 Y \\ h \sim H, k \sim K}} b(h) g(k) e(\theta h k p), \quad S_{2}(\theta)=\sum_{N-Y<p_{1} \leq N} e\left(p_{1} \theta\right) .
$$

In order to prove

$$
\sum_{N<n \leq N+A}\left|\int_{E_{2}} S_{1}(\theta) S_{2}(\theta) e(-n \theta) d \theta\right|^{2} \ll A Y^{2} \log ^{-52 B} N
$$

it suffices to prove

$$
\max _{\substack{q \leq Q \\(a, q)=1}} \int_{\Omega(a, q)}\left|S_{1}(\alpha)\right|^{2} d \alpha \ll Y \log ^{-120 B} N .
$$

Using the argument of Lemma 15, we can deal with the part with $(h k p, q)>\Gamma=\max \left(q^{2 \varepsilon}, \log ^{260 B} N\right)$ in $S_{1}(\alpha)$. Since $(h k p, q) \leq \Gamma$ and $b(h) g(k) \neq 0$ imply that $(h k p, q)=1$, we need to prove

$$
\max _{\substack{q \leq Q \\(a, q)=1}} \int_{\Omega(a, q)}\left|S_{3}(\alpha)\right|^{2} d \alpha \ll Y \log ^{-120 B} N
$$

where

$$
S_{3}(\alpha)=\sum_{\substack{m h k<2 Y \\ h \sim H, k \sim K \\(m h k, q)=1}} \Lambda(m) b(h) g(k) e\left(\frac{a m h k}{q}\right) e(\beta m h k) .
$$

We have

$$
\begin{aligned}
S_{3}(\alpha)= & \frac{1}{\varphi(q)} \sum_{\chi(\bmod q)} \chi(a) \tau(\bar{\chi}) \\
& \times \sum_{\substack{m h k \leq 2 Y \\
h \sim H, k \sim K}} \Lambda(m) \chi(m) b(h) \chi(h) g(k) \chi(k) e(\beta m h k)
\end{aligned}
$$




$$
\begin{aligned}
= & \frac{1}{\varphi(q)} \sum_{\chi(\bmod q)} \chi(a) \tau(\bar{\chi}) W(\chi, \beta) \\
& +\frac{\mu(q)}{\varphi(q)} \sum_{\substack{h \sim H \\
(h, q)=1}} \frac{b(h)}{h} \sum_{\substack{k \sim K \\
(k, q)=1}} \frac{g(k)}{k} \sum_{r \leq 2 Y} e(\beta r),
\end{aligned}
$$

where

$$
\begin{aligned}
W(\chi, \beta)= & \sum_{\substack{m h k \leq 2 Y \\
h \sim H, k \sim K}} \Lambda(m) \chi(m) b(h) \chi(h) g(k) \chi(k) e(\beta m h k) \\
& -E_{0} \sum_{\substack{h \sim H \\
(h, q)=1}} \frac{b(h)}{h} \sum_{\substack{k \sim K \\
(k, q)=1}} \frac{g(k)}{k} \sum_{r \leq 2 Y} e(\beta r) .
\end{aligned}
$$

Now,

$$
W(\chi, \beta)=\sum_{r \leq 2 Y}\left(\lambda(r) \chi(r)-E_{0} I\right) e(\beta r),
$$

where

$$
\lambda(r)=\sum_{\substack{m h k=r \\ h \sim H, k \sim K}} \Lambda(m) b(h) g(k), \quad I=\sum_{\substack{h \sim H \\(h, q)=1}} \frac{b(h)}{h} \sum_{\substack{k \sim K \\(k, q)=1}} \frac{g(k)}{k} .
$$

By the argument of Lemma 15, we need to prove

$$
\frac{1}{\varphi^{2}(q)} \int_{\Phi(q)}\left|\sum_{\chi(\bmod q)} \chi(a) \tau(\bar{\chi}) W(\chi, \beta)\right|^{2} d \beta \ll Y \log ^{-120 B} N .
$$

By the explanation in Section 2,

$$
\begin{aligned}
\frac{1}{\varphi^{2}(q)} \int_{\Phi(q)}\left|\sum_{\chi(\mathrm{bad})} \chi(a) \tau(\bar{\chi}) W(\chi, \beta)\right|^{2} d \beta & \\
& \ll \frac{q}{\varphi^{2}(q)} \log ^{\frac{64 E}{\varepsilon}} N \sum_{\chi(\mathrm{bad})-\frac{1}{2}} \int_{\frac{1}{2}}|W(\chi, \beta)|^{2} d \beta \\
& \ll \frac{Y}{q} \log ^{\frac{129 E}{\varepsilon}} N \ll \frac{Y}{\log ^{E} N} .
\end{aligned}
$$

Since

$$
\begin{aligned}
\frac{1}{\varphi^{2}(q)} \int_{\Phi(q)}\left|\sum_{\chi(\text { good })} \chi(a) \tau(\bar{\chi}) W(\chi, \beta)\right|^{2} d \beta & \\
& \ll \frac{q^{2}}{\varphi^{2}(q)} \sum_{\chi(\text { good })} \int_{-\frac{1}{q Q}}^{\frac{1}{q Q}}|W(\chi, \beta)|^{2} d \beta,
\end{aligned}
$$


it suffices to prove

$$
\sum_{\chi(\text { good })} \int_{-\frac{1}{q Q}}^{\frac{1}{q Q}}|W(\chi, \beta)|^{2} d \beta \ll \frac{Y}{\log ^{E} N} .
$$

By the explanation of Lemma 15, we only estimate for $\eta=q Q / Y$ the quantity

$$
\Sigma_{2}=\frac{1}{(q Q)^{2}} \sum_{\chi(\text { good })} \int_{Y}^{3 Y}\left|\sum_{x<r \leq x+\eta x}\left(\lambda(r) \chi(r)-E_{0} I\right)\right|^{2} d x .
$$

Perron's formula yields

$$
\sum_{x<r \leq x+\eta x} \lambda(r) \chi(r)=\frac{1}{2 \pi i} \int_{b-i Y}^{b+i Y} G(s, \chi) \frac{(1+\eta)^{s}-1}{s} x^{s} d s+O\left(Y^{\varepsilon}\right) .
$$

If $s=b+i t,|t| \leq 3 Y$ and $\chi$ is a good character $\bmod q$,

$$
\sum_{m \sim M} \frac{\Lambda(m) \chi(m)}{m^{s}}=E_{0} \frac{\left(c_{2} M\right)^{1-s}-\left(c_{1} M\right)^{1-s}}{1-s}+O\left(\log ^{-\frac{2 E}{\delta^{2}}} Y\right)
$$

(see (4)).

By the argument of Lemma 15, we have

$$
\begin{aligned}
\frac{1}{2 \pi i} \int_{b-i T_{0}}^{b+i T_{0}} G(s, \chi) & \frac{(1+\eta)^{s}-1}{s} x^{s} d s \\
= & \eta E_{0} \frac{1}{2 \pi i} \int_{b-i T_{0}}^{b+i T_{0}} H(s, \chi) K(s, \chi) \frac{\left(c_{2} M\right)^{1-s}-\left(c_{1} M\right)^{1-s}}{1-s} x^{s} d s \\
& +O\left(S_{1}\right)+O\left(S_{2}\right),
\end{aligned}
$$

where

$$
\begin{aligned}
& S_{1}=\eta Y(\log N)^{-\frac{2 E}{\delta^{2}}} \int_{-T_{0}}^{T_{0}}|H(b+i t, \chi) K(b+i t, \chi)| d t, \\
& S_{2}=\eta^{2} Y \int_{-T_{0}}^{T_{0}}|H(b+i t, \chi) K(b+i t, \chi)| d t .
\end{aligned}
$$

By Lemma 4 , the contribution of $S_{1}$ to (37) is

$$
\begin{aligned}
& \ll Y(\log N)^{-\frac{4 E}{\delta^{2}}} \sum_{\chi(\bmod q)} T_{0} \int_{-T_{0}}^{T_{0}}|H(b+i t, \chi) K(b+i t, \chi)|^{2} d t \\
& \ll Y T_{0}(\log N)^{-\frac{4 E}{\delta^{2}}}\left(1+\frac{q T_{0}}{H K}\right) \log ^{6} N \ll Y \log ^{-2 E} N .
\end{aligned}
$$


The contribution of $S_{2}$ to (37) is

$$
\begin{aligned}
& \ll \frac{1}{(q Q)^{2}} \cdot Y\left(\frac{q Q}{Y} \cdot q Q\right)^{2} \sum_{\chi(\bmod q)} T_{0} \int_{-T_{0}}^{T_{0}}|H(b+i t, \chi) K(b+i t, \chi)|^{2} d t \\
& \ll Y T_{0}\left(\frac{q Q}{Y}\right)^{2}\left(1+\frac{q T_{0}}{H K}\right) \log ^{6} N \ll Y^{1-\varepsilon} .
\end{aligned}
$$

By Perron's formula again,

$$
\begin{aligned}
\eta E_{0} \cdot \frac{1}{2 \pi i} \int_{b-i T_{0}}^{b+i T_{0}} H(s, \chi) K(s, \chi) \frac{\left(c_{2} M\right)^{1-s}-\left(c_{1} M\right)^{1-s}}{1-s} x^{s} d s \\
=\eta x \cdot E_{0} \cdot \sum_{h \sim H} \frac{b(h) \chi(h)}{h} \sum_{k \sim K} \frac{g(k) \chi(k)}{k} \\
+O\left(\frac{\eta x E_{0} \log ^{2} Y}{T_{0}}\right)+O\left(\frac{\eta x E_{0} Y^{\varepsilon}}{H K}\right) \\
=\eta x \cdot E_{0} I+O\left(\frac{\eta x E_{0} \log ^{2} Y}{T_{0}}\right)+O\left(\frac{\eta x E_{0} Y^{\varepsilon}}{H K}\right) .
\end{aligned}
$$

The contribution of the term $O\left(\eta x E_{0} \log ^{2} Y / T_{0}\right)$ to (37) is

$$
\ll \frac{1}{(q Q)^{2}} \cdot Y\left(\frac{q Q \log ^{2} Y}{T_{0}}\right)^{2} \ll Y \log ^{-E} N .
$$

The contribution of the term $O\left(\eta x E_{0} Y^{\varepsilon} /(H K)\right)$ to (37) is

$$
\ll \frac{1}{(q Q)^{2}} \cdot Y\left(\frac{q Q Y^{\varepsilon}}{H K}\right)^{2} \ll Y^{1-\varepsilon} .
$$

Combining the above, we have

(38) $\quad \Sigma_{2} \ll \frac{\log ^{2} N}{(q Q)^{2}}$

$$
\times \max _{T_{0} \leq|T| \leq Y} \sum_{\chi(\text { good })} \int_{Y}^{3 Y}\left|\int_{b+i T}^{b+2 i T} G(s, \chi) \frac{(1+\eta)^{s}-1}{s} x^{s} d s\right|^{2} d x+O\left(Y \log ^{-E} N\right) .
$$

By the argument of Lemma 15, we have

$$
\begin{aligned}
\Sigma_{2} \ll & \frac{Y^{3} \log ^{4} N}{(q Q)^{2}} \cdot \max _{T_{0} \leq T \leq Y} \min ^{2}\left(\eta, \frac{1}{T}\right) \sum_{\chi(\text { good })} \int_{T}^{2 T}|G(b+i t, \chi)|^{2} d t \\
& +O\left(Y \log ^{-E} N\right) .
\end{aligned}
$$

By the assumption in Lemma $18, \Sigma_{2} \ll Y \log ^{-E} N$. Hence, (33) holds. Then 
for $N<n \leq N+A$, except for $O\left(A \log ^{-B} N\right)$ values,

$$
\int_{E_{2}} S_{1}(\theta) S_{2}(\theta) e(-n \theta) d \theta=O\left(Y \log ^{-B} N\right) .
$$

If $\theta=a / q+\beta \in E_{1}$, we have

$$
S_{1}(\theta)=\sum_{\substack{p d \leq 2 Y \\ d \sim D}} l(d) e(\theta p d)
$$

where $D=H K$ and

$$
l(d)=\sum_{\substack{h k=d \\ h \sim H, k \sim K}} b(h) g(k) .
$$

Hence

$$
\begin{aligned}
S_{1}(\theta) & =\sum_{d \sim D} l(d) \sum_{p \leq 2 Y / d} e\left(\frac{a p d}{q}+\beta p d\right) \\
& =\sum_{d \sim D} l(d) \sum_{\substack{p \leq 2 Y / d \\
(p, q)=1}} e\left(\frac{a p d}{q}+\beta p d\right)+O\left(Y^{1-\varepsilon}\right),
\end{aligned}
$$

where

$$
\sum_{\substack{p \leq 2 Y / d \\(p, q)=1}} e\left(\frac{a p d}{q}+\beta p d\right)=\sum_{\substack{l=1 \\(l, q)=1}}^{q} e\left(\frac{a d l}{q}\right) \sum_{\substack{p \leq 2 Y / d \\ p \equiv l(\bmod q)}} e(\beta p d) .
$$

From the prime number theorem in the arithmetic progression, it follows that

$$
\begin{aligned}
\sum_{\substack{p \leq 2 Y / d \\
p \equiv l(\bmod q)}} e(\beta p d) & =\int_{2}^{2 Y / d} e(\beta d t) d \pi(t ; l, q) \\
& =\frac{1}{\varphi(q)} \int_{2}^{2 Y / d} \frac{e(\beta d t)}{\log t} d t+O\left(\frac{Y}{d \log ^{6 E} N}\right) \\
& =\frac{1}{\varphi(q)} \cdot \frac{1}{d} \cdot \int_{2 d}^{2 Y} \frac{e(\beta u)}{\log (u / d)} d u+O\left(\frac{Y}{d \log ^{6 E} N}\right) .
\end{aligned}
$$

Note that $q \leq \log ^{E} N$ and $(d, q)>1$ imply $l(d)=0$. So, $(d, q)=1$ can be assumed. Consequently,

$$
\sum_{\substack{p \leq 2 Y / d \\(p, q)=1}} e\left(\frac{a p d}{q}+\beta p d\right)
$$




$$
\begin{aligned}
& =\frac{1}{\varphi(q)} \cdot \frac{1}{d} \sum_{\substack{l=1 \\
(l, q)=1}}^{q} e\left(\frac{a d l}{q}\right) \int_{2 d}^{2 Y} \frac{e(\beta u)}{\log (u / d)} d u+O\left(\frac{Y}{d \log ^{5 E} N}\right) \\
& =\frac{\mu(q)}{\varphi(q)} \cdot \frac{1}{d} \sum_{2 d \leq r \leq 2 Y} \frac{e(\beta r)}{\log (r / d)}+O\left(\frac{Y}{d \log ^{5 E} N}\right) .
\end{aligned}
$$

Therefore

$$
S_{1}(\theta)=\frac{\mu(q)}{\varphi(q)} \sum_{d \sim D} \frac{l(d)}{d} \sum_{2 d \leq r \leq 2 Y} \frac{e(\beta r)}{\log (r / d)}+O\left(\frac{Y}{\log ^{4 E} N}\right) .
$$

Now, set

$$
\begin{aligned}
\Sigma_{3}= & \int_{E_{1}} S_{1}(\theta) S_{2}(\theta) e(-n \theta) d \theta \\
= & \sum_{q \leq \log ^{E}} \sum_{N}^{q} \int_{\substack{a=1 \\
(a, q)=1}}^{\log ^{2 E} \log ^{2 E}{ }_{N /(q Y)}^{N /(q Y)}} S_{1}\left(\frac{a}{q}+\beta\right) S_{2}\left(\frac{a}{q}+\beta\right) \\
& \times e\left(-\left(\frac{a}{q}+\beta\right) n\right) d \beta \\
= & \sum_{q \leq \log ^{E} N} \frac{\mu^{2}(q)}{\varphi^{2}(q)} \sum_{\substack{a=1 \\
(a, q)=1}}^{q} e\left(-\frac{a n}{q}\right) \int_{-\log ^{2 E}}^{\log ^{2 E}{ }_{N /(q Y)}} \sum_{d \sim D} \frac{l(d)}{d} \\
& \times \sum_{2 d \leq r \leq 2 Y} \frac{e(\beta r)}{\log (r / d)} \cdot \frac{1}{\log N} \sum_{N-Y \leq s \leq N} e(\beta s) e(-n \beta) d \beta \\
& +O\left(\frac{Y}{\log ^{B} N}\right) .
\end{aligned}
$$

Since

$$
\begin{aligned}
\int_{-\log ^{2 E}}^{\log ^{2 E}} \int_{N /(q Y)}^{N /(q Y)} & \sum_{d \sim D} \frac{l(d)}{d} \sum_{2 d \leq r \leq 2 Y} \frac{e(\beta r)}{\log (r / d)} \sum_{N-Y<s \leq N} e(\beta s) e(-n \beta) d \beta \\
= & \int_{-\frac{1}{2}}^{\frac{1}{2}} \sum_{d \sim D} \frac{l(d)}{d} \sum_{2 d \leq r \leq 2 Y} \frac{e(\beta r)}{\log (r / d)} \sum_{N-Y<s \leq N} e(\beta s) e(-n \beta) d \beta \\
& +O\left(\frac{q Y}{\log ^{2 E} N}\right)
\end{aligned}
$$




$$
\begin{aligned}
& =\sum_{d \sim D} \frac{l(d)}{d} \sum_{2 d \leq r \leq 2 Y} \frac{1}{\log (r / d)} \sum_{\substack{N-Y<s \leq N \\
r+s=n}} 1+O\left(\frac{q Y}{\log ^{2 E} N}\right) \\
& =\sum_{d \sim D} \frac{l(d)}{d} \sum_{2 d \leq r \leq Y} \frac{1}{\log (r / d)}+O\left(\frac{q Y}{\log ^{2 E} N}\right),
\end{aligned}
$$

we have

$$
\begin{aligned}
\Sigma_{3}= & \frac{1}{\log N} \sum_{q \leq \log ^{E} N} \frac{\mu^{2}(q)}{\varphi^{2}(q)} \sum_{\substack{a=1 \\
(a, q)=1}}^{q} e\left(-\frac{a n}{q}\right) \sum_{d \sim D} \frac{l(d)}{d} \sum_{2 d \leq r \leq Y} \frac{1}{\log (r / d)} \\
& +O\left(\frac{Y}{\log ^{B} N}\right) .
\end{aligned}
$$

By the argument in Lemmas 11 and 12 of [7], we find that for $N<n \leq N+A$, except for $O\left(A \log ^{-B} N\right)$ values,

$$
\sum_{q \leq \log ^{E} N} \frac{\mu^{2}(q)}{\varphi^{2}(q)} \sum_{\substack{a=1 \\(a, q)=1}}^{q} e\left(-\frac{a n}{q}\right)=C(n)+O\left(\frac{1}{\log ^{2 B} N}\right) .
$$

Hence,

$$
\begin{aligned}
& \Sigma_{3}=C(n) \cdot \frac{Y}{\log N}\left(1+O\left(\frac{1}{\log N}\right)\right) \sum_{d \sim D} \frac{l(d)}{d \log (Y / d)}+O\left(\frac{Y}{\log ^{B} N}\right) \\
& =\frac{C(n)}{\log N}\left(1+O\left(\frac{1}{\log N}\right)\right) \sum_{\substack{h \sim H \\
k \sim K}} b(h) g(k) \sum_{Y /(h k)<p \leq 2 Y /(h k)} 1+O\left(\frac{Y}{\log ^{B} N}\right) .
\end{aligned}
$$

The proof of Lemma 18 is complete.

Lemma 19. Suppose that $Y^{\frac{119}{135}} \ll M \ll Y^{1-\delta}$ and $0 \leq a(m)=O(1)$. If $m$ has a prime factor $<Y^{\delta}, a(m)=0$. Then for $N<n \leq N+A$, except for $O\left(A \log ^{-B} N\right)$ values, we have

$$
\begin{aligned}
\sum_{\substack{m p+p_{1}=n \\
N-Y<p_{1} \leq N \\
m p \leq 2 Y \\
m \sim M}} a(m)= & \frac{C(n)}{\log N}\left(1+O\left(\frac{1}{\log N}\right)\right) \sum_{m \sim M} a(m) \sum_{Y / m<p \leq 2 Y / m} 1 \\
& +O\left(Y \log ^{-B} N\right) .
\end{aligned}
$$

Pr o of. This follows from Lemmas 5 and 18. 
8. Buchstab's function. We define $w(u)$ as the continuous solution of the equations

$$
\begin{cases}w(u)=1 / u, & 1 \leq u \leq 2, \\ (u w(u))^{\prime}=w(u-1), & u>2 .\end{cases}
$$

$w(u)$ is called Buchstab's function and plays an important role in finding asymptotic formulas in the sieve method. In particular,

$$
\begin{aligned}
w(u)= & \frac{1+\log (u-1)}{u}, \quad 2 \leq u \leq 3 ; \\
w(u)= & \frac{1+\log (u-1)}{u}+\frac{1}{u} \int_{2}^{u-1} \frac{\log (t-1)}{t} d t, \quad 3 \leq u \leq 4 ; \\
w(u)= & \frac{1+\log (u-1)}{u}+\frac{1}{u} \int_{2}^{u-1} \frac{\log (t-1)}{t} d t \\
& +\frac{1}{u} \int_{3}^{u-1} \frac{d t}{t} \int_{2}^{t-1} \frac{\log (s-1)}{s} d s, \quad 4 \leq u \leq 5 .
\end{aligned}
$$

LEMma 20. For the function $w(u)$, we have the following bounds:

(i) $w(u) \geq 0.5607$ for $u \geq 2.47$,

(ii) $w(u) \leq 0.5644$ for $u \geq 3$,

(iii) $0.5612 \leq w(u) \leq 0.5617$ for $u \geq 4$.

Proof. It is easy to see that $0.5 \leq w(u) \leq 1$ for $1 \leq u \leq 2$. Then we employ induction.

Suppose that $0.5 \leq w(u) \leq 1$ for $1 \leq k \leq u \leq k+1$. If $k+1 \leq u \leq k+2$, then (40) yields

$$
u w(u)=(k+1) w(k+1)+\int_{k}^{u-1} w(t) d t .
$$

Hence, $0.5 \leq w(u) \leq 1$ for $k+1 \leq u \leq k+2$. By induction, we obtain $0.5 \leq w(u) \leq 1$ for $u \geq 1$.

If $u>2$, (40) yields

$$
w^{\prime}(u)=\frac{w(u-1)-w(u)}{u} .
$$

If $2 \leq u \leq 3$, by calculation, we have

$$
\max _{0 \leq k \leq 10^{4}} w\left(2+10^{-4} k\right) \leq 0.56716 .
$$

From (42) and $0.5 \leq w(u) \leq 1$ for $u \geq 1$, it follows that $\left|w^{\prime}(u)\right| \leq \frac{1}{4}$ if $u>2$. Using Lagrange's mean value theorem, we have $w(u) \leq 0.5672$ for $2 \leq u \leq 3$. By induction, we obtain $0.5 \leq w(u) \leq 0.5672$ for $u \geq 2$. 
If $3 \leq u \leq 4$, by calculation, we have

$$
\max _{0 \leq k \leq 10^{4}} w\left(3+10^{-4} k\right) \leq 0.56439, \quad \min _{0 \leq k \leq 10^{4}} w\left(3+10^{-4} k\right) \geq 0.56081 .
$$

From (42) and $0.5 \leq w(u) \leq 0.5672$ for $u \geq 2$, it follows that $\left|w^{\prime}(u)\right| \leq$ 0.0224 if $u>3$. The above discussion implies that $0.5607 \leq w(u) \leq 0.5644$ for $u \geq 3$. By the same discussion we can also get $0.5607 \leq w(u)$ for $u \geq 2.47$.

If $4 \leq u \leq 5$, by calculation, we have

$$
\max _{0 \leq k \leq 10^{4}} w\left(4+10^{-4} k\right) \leq 0.5616, \quad \min _{0 \leq k \leq 10^{4}} w\left(4+10^{-4} k\right) \geq 0.5613 .
$$

The above discussion and the fact that $\left|w^{\prime}(u)\right| \leq 0.0224$ for $u>3$ imply that $0.5612 \leq w(u) \leq 0.5617$ for $u \geq 4$.

Gathering together the above discussion, we get Lemma 20.

Lemma 21. Suppose that $\mathcal{E}=\{n: t<n \leq 2 t\}$ and $z \leq t$. Let

$$
P(z)=\prod_{p<z} p .
$$

Then for sufficiently large $t$ and $z$, we have

$$
S(\mathcal{E}, z)=\sum_{\substack{t<n \leq 2 t \\(n, P(\bar{z}))=1}} 1=\left(w\left(\frac{\log t}{\log z}\right)+O(\varepsilon)\right) \frac{t}{\log z} .
$$

Proof. See Lemma 5 of [9]. If $(2 t)^{\frac{1}{2}}<z \leq t$, it is the prime number theorem.

9. Sieve method. Let $n$ be an even number, $N<n \leq N+A$, and set

$$
T(n)=\sum_{\substack{p_{1}+p_{2}=n \\ N-Y<p_{2} \leq N \\ p_{1} \leq 2 Y}} 1
$$

We proceed to show that

$$
T(n) \geq 0.011 \cdot \frac{C(n) Y}{\log Y \log N},
$$

where

$$
C(n)=\prod_{p \nmid n}\left(1-\frac{1}{(p-1)^{2}}\right) \prod_{p \mid n}\left(1+\frac{1}{p-1}\right) .
$$

Set

$$
\begin{gathered}
\mathcal{A}=\{a: a=n-p, N-Y<p \leq N\}, \quad \mathcal{P}=\{p: p \nmid n\}, \\
P(z, n)=\prod_{\substack{p<z \\
p \in \mathcal{P}}} p, \quad S(\mathcal{A}, z)=\sum_{\substack{a \in \mathcal{A} \\
(a, P(z, n))=1}} 1 .
\end{gathered}
$$


Then

$$
T(n)=S\left(\mathcal{A},(2 Y)^{\frac{1}{2}}\right)+O\left(Y^{\frac{1}{2}}\right) .
$$

Buchstab's identity yields

$$
\begin{aligned}
S\left(\mathcal{A},(2 Y)^{\frac{1}{2}}\right)= & S\left(\mathcal{A}, Y^{\frac{16}{135}}\right)-\sum_{Y^{\frac{16}{135}}<p \leq(2 Y)^{\frac{1}{2}}} S\left(\mathcal{A}_{p}, p\right) \\
= & S\left(\mathcal{A}, Y^{\frac{16}{135}}\right)-\sum_{Y^{\frac{16}{135}}<p \leq(2 Y)^{\frac{1}{2}}} S\left(\mathcal{A}_{p}, Y^{\frac{16}{135}}\right) \\
& +\sum_{Y^{\frac{16}{135}}<p \leq(2 Y)^{\frac{1}{2}}} \sum_{Y^{\frac{16}{135}}<q<\min \left(p,\left(\frac{2 Y}{p}\right)^{\frac{1}{2}}\right)} S\left(\mathcal{A}_{p q}, q\right) .
\end{aligned}
$$

The following lemmas always concern $N<n \leq N+A$, except for $O\left(A \log ^{-B} N\right)$ values. Let

$$
\mathcal{B}=\{n: Y<n \leq 2 Y\} .
$$

LEMma 22. We have

$$
S\left(\mathcal{A}, Y^{\frac{16}{135}}\right) \geq 4.735124 \cdot \frac{C(n) Y}{\log Y \log N} .
$$

Proof. We have

$$
S\left(\mathcal{A}, Y^{\frac{16}{135}}\right)=S\left(\mathcal{A}, Y^{\delta}\right)-\sum_{Y^{\delta}<p \leq Y \frac{16}{135}} S\left(\mathcal{A}_{p}, p\right) .
$$

Let $X=Y / \log N$ and

$$
\omega(d)=\left\{\begin{array}{ll}
d / \varphi(d), & (d, n)=1, \\
0, & (d, n)>1,
\end{array} \quad \widetilde{r}(\mathcal{A}, d)=\left|\mathcal{A}_{d}\right|-\frac{X}{\varphi(d)} .\right.
$$

By the argument in Lemma 11 of [8] (see also Theorem 2 on p. 164 and (40) on p. 171 of [15]),

$$
W(z)=\prod_{p<z}\left(1-\frac{\omega(p)}{p}\right)=(1+O(\varepsilon)) C(n) \frac{e^{-\gamma}}{\log z},
$$

where $\gamma$ is Euler's constant.

Let $z=Y^{\delta}$ and $D=Y^{\frac{19}{36}}$. Applying Iwaniec's sieve method (see Theorem 1 of [6]), we have

$$
S\left(\mathcal{A}, Y^{\delta}\right) \geq \frac{C(n) Y}{\log z \log N} f\left(\frac{\log D}{\log z}\right)-O\left(\frac{\delta C(n) Y}{\log ^{2} N}\right)-R^{-},
$$

where

$$
R^{-}=\sum_{\substack{m \leq Y^{\frac{19}{36}} \\(m, n)=1}} a(m) \widetilde{r}(\mathcal{A}, m)
$$


Lemma 17 yields $R^{-}=O\left(Y \log ^{-5} N\right)$. By Theorem 8 on p. 181 of [15], we have

$$
f\left(\frac{\log D}{\log z}\right)=e^{-\gamma}+O\left(\varepsilon^{2}\right)
$$

where $\gamma$ is Euler's constant. Thus,

$$
S\left(\mathcal{A}, Y^{\delta}\right) \geq \frac{e^{-\gamma}}{\delta} \cdot \frac{C(n) Y}{\log Y \log N}+O\left(\frac{\delta C(n) Y}{\log ^{2} N}\right) .
$$

In the same way,

$$
\begin{aligned}
S\left(\mathcal{A}, Y^{\delta}\right) & \leq \frac{C(n) Y}{\log z \log N} \cdot F\left(\frac{\log D}{\log z}\right)+O\left(\frac{\delta C(n) Y}{\log ^{2} N}\right)+\sum_{\substack{m \leq Y^{\frac{19}{36}} \\
(m, n)=1}} b(m) \widetilde{r}(\mathcal{A}, m) \\
& \leq \frac{e^{-\gamma}}{\delta} \cdot \frac{C(n) Y}{\log Y \log N}+O\left(\frac{\delta C(n) Y}{\log ^{2} N}\right) .
\end{aligned}
$$

So, we have

$$
S\left(\mathcal{A}, Y^{\delta}\right)=\frac{e^{-\gamma}}{\delta} \cdot \frac{C(n) Y}{\log Y \log N}+O\left(\frac{\delta C(n) Y}{\log ^{2} N}\right)
$$

Now,

$$
\sum_{Y^{\delta}<p \leq Y \frac{16}{135}} S\left(\mathcal{A}_{p}, p\right)=\sum_{p q+p_{1}=n} 1
$$

where $p q \leq 2 Y, N-Y<p_{1} \leq N, Y^{\delta}<p \leq Y^{\frac{16}{135}}$, and the least prime factor of $q$ is greater than $p$. Using Lemmas 19 and 21 and the prime number theorem, we have the asymptotic formula

$$
\begin{aligned}
& \sum_{Y^{\delta}<p \leq Y}^{\frac{16}{135}} S\left(\mathcal{A}_{p}, p\right) \\
= & \frac{C(n)}{\log N} \sum_{Y^{\delta}<p \leq Y Y^{\frac{16}{135}}} S\left(\mathcal{B}_{p}, p\right)+O\left(\frac{\delta C(n) Y}{\log ^{2} N}\right) \\
= & \frac{C(n) Y}{\log N} \sum_{Y^{\delta}<p \leq Y \frac{16}{135}} \frac{1}{p \log p} w\left(\frac{\log (Y / p)}{\log p}\right)+O\left(\frac{\delta C(n) Y}{\log ^{2} N}\right)
\end{aligned}
$$




$$
\begin{aligned}
& =\frac{C(n) Y}{\log Y \log N} \int_{\delta}^{\frac{16}{135}} \frac{1}{t^{2}} w\left(\frac{1-t}{t}\right) d t+O\left(\frac{\delta C(n) Y}{\log ^{2} N}\right) \\
& =\frac{C(n) Y}{\log Y \log N} \int_{\frac{135}{16}}^{\frac{1}{\delta}} w(u-1) d u+O\left(\frac{\delta C(n) Y}{\log ^{2} N}\right) \\
& =\frac{C(n) Y}{\log Y \log N}\left\{\frac{1}{\delta} w\left(\frac{1}{\delta}\right)-\frac{135}{16} w\left(\frac{135}{16}\right)\right\}+O\left(\frac{\delta C(n) Y}{\log ^{2} N}\right) \\
& =\frac{e^{-\gamma}}{\delta} \cdot \frac{C(n) Y}{\log Y \log N}-\frac{135}{16} w\left(\frac{135}{16}\right) \cdot \frac{C(n) Y}{\log Y \log N}+O\left(\frac{\delta C(n) Y}{\log ^{2} N}\right),
\end{aligned}
$$

since

$$
w\left(\frac{1}{\delta}\right)=e^{-\gamma}+O\left(\varepsilon^{2}\right)
$$

(see Lemma 12 on p. 179 of [15]). Hence,

$$
S\left(\mathcal{A}, Y^{\frac{16}{135}}\right)=\frac{135}{16} w\left(\frac{135}{16}\right) \cdot \frac{C(n) Y}{\log Y \log N}+O\left(\frac{\delta C(n) Y}{\log ^{2} N}\right) .
$$

By Lemma 20, we get

$$
\begin{aligned}
S\left(\mathcal{A}, Y^{\frac{16}{135}}\right) & \geq \frac{135}{16} \cdot 0.5612 \cdot \frac{C(n) Y}{\log Y \log N}+O\left(\frac{\delta C(n) Y}{\log ^{2} N}\right) \\
& \geq 4.735124 \cdot \frac{C(n) Y}{\log Y \log N} .
\end{aligned}
$$

The proof of Lemma 22 is complete.

Lemma 23. We have

$$
\sum_{Y^{\frac{16}{135}}<p \leq(2 Y)^{\frac{1}{2}}} S\left(\mathcal{A}_{p}, Y^{\frac{16}{135}}\right) \leq 6.822470 \cdot \frac{C(n) Y}{\log Y \log N} .
$$

Proof. Buchstab's identity yields

$$
\begin{aligned}
& \quad \sum_{Y^{\frac{16}{135}}<p \leq(2 Y)^{\frac{1}{2}}} S\left(\mathcal{A}_{p}, Y^{\frac{16}{135}}\right) \\
& =\sum_{Y^{\frac{16}{135}}<p \leq(2 Y)^{\frac{1}{2}}} S\left(\mathcal{A}_{p}, Y^{\delta}\right)-\sum_{Y^{\frac{16}{135}<p \leq(2 Y)^{\frac{1}{2}}}} \sum_{Y^{\delta}<q<Y^{\frac{16}{135}}} S\left(\mathcal{A}_{p q}, q\right) .
\end{aligned}
$$

Using Lemma 17, in the same way as in Lemma 22, we have 


$$
\begin{aligned}
& \sum_{Y^{\frac{16}{135}<p \leq(2 Y)^{\frac{1}{2}}} S\left(\mathcal{A}_{p}, Y^{\delta}\right)=} \sum_{Y^{\frac{16}{135}<p \leq(2 Y)^{\frac{1}{2}}} \frac{e^{-\gamma}}{\delta} \cdot \frac{1}{p} \cdot \frac{C(n) Y}{\log Y \log N}} \\
&+O\left(\frac{\delta C(n) Y}{\log ^{2} N}\right) \\
&= \frac{e^{-\gamma}}{\delta} \cdot \frac{C(n) Y}{\log Y \log N} \int_{\frac{16}{135}}^{\frac{1}{2}} \frac{d t}{t}+O\left(\frac{\delta C(n) Y}{\log ^{2} N}\right) .
\end{aligned}
$$

Using Lemmas 19 and 21, in the same way as in Lemma 22, we have

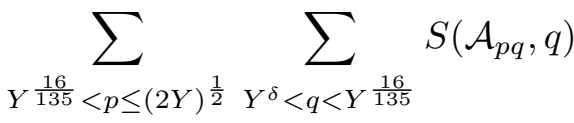

$$
\begin{aligned}
& =\frac{C(n)}{\log N} \sum_{Y^{\frac{16}{135}}<p \leq(2 Y)^{\frac{1}{2}}} \sum_{Y^{\delta}<q \leq Y} S_{\frac{16}{135}} S\left(\mathcal{B}_{p q}, q\right)+O\left(\frac{\delta C(n) Y}{\log ^{2} N}\right) \\
& =\frac{C(n) Y}{\log N} \sum_{Y^{\frac{16}{135}}<p \leq(2 Y)^{\frac{1}{2}}} \sum_{Y^{\delta}<q \leq Y} \frac{1}{\frac{16}{135}} \frac{1}{p q \log q} w\left(\frac{\log (Y /(p q))}{\log q}\right) \\
& +O\left(\frac{\delta C(n) Y}{\log ^{2} N}\right) \\
& =\frac{C(n) Y}{\log Y \log N} \int_{\frac{16}{135}}^{\frac{1}{2}} \frac{d t}{t} \int_{\delta}^{\frac{16}{135}} \frac{1}{u^{2}} w\left(\frac{1-t-u}{u}\right) d u+O\left(\frac{\delta C(n) Y}{\log ^{2} N}\right) \\
& =\frac{C(n) Y}{\log Y \log N} \int_{\frac{16}{135}}^{\frac{1}{2}} \frac{d t}{t(1-t)} \int_{\frac{135}{16}(1-t)}^{\frac{1}{\delta}(1-t)} w(r-1) d r+O\left(\frac{\delta C(n) Y}{\log ^{2} N}\right) \\
& =\frac{1}{\delta} \cdot \frac{C(n) Y}{\log Y \log N} \int_{\frac{16}{135}}^{\frac{1}{2}} \frac{1}{t} w\left(\frac{1}{\delta}(1-t)\right) d t \\
& -\frac{135}{16} \cdot \frac{C(n) Y}{\log Y \log N} \int_{\frac{16}{135}}^{\frac{1}{2}} \frac{1}{t} w\left(\frac{135}{16}(1-t)\right) d t+O\left(\frac{\delta C(n) Y}{\log ^{2} N}\right) \\
& =\frac{e^{-\gamma}}{\delta} \cdot \frac{C(n) Y}{\log Y \log N} \int_{\frac{16}{135}}^{\frac{1}{2}} \frac{d t}{t}-\frac{135}{16} \cdot \frac{C(n) Y}{\log Y \log N} \int_{\frac{16}{135}}^{\frac{1}{2}} \frac{1}{t} w\left(\frac{135}{16}(1-t)\right) d t \\
& +O\left(\frac{\delta C(n) Y}{\log ^{2} N}\right) \text {. }
\end{aligned}
$$


Gathering together the above discussion and applying Lemma 20, we have

$$
\begin{aligned}
\sum_{Y^{\frac{16}{135}<p \leq(2 Y)^{\frac{1}{2}}}} S\left(\mathcal{A}_{p}, Y^{\frac{16}{135}}\right) \\
=\frac{135}{16} \cdot \frac{C(n) Y}{\log Y \log N} \int_{\frac{16}{135}}^{\frac{1}{2}} \frac{1}{t} w\left(\frac{135}{16}(1-t)\right) d t+O\left(\frac{\delta C(n) Y}{\log ^{2} N}\right) \\
\leq 0.5617 \cdot \frac{135}{16} \cdot \log \left(\frac{135}{32}\right) \cdot \frac{C(n) Y}{\log Y \log N}+O\left(\frac{\delta C(n) Y}{\log ^{2} N}\right) \\
\leq 6.822470 \cdot \frac{C(n) Y}{\log Y \log N} .
\end{aligned}
$$

The proof of Lemma 23 is complete.

We now set

$$
\begin{aligned}
\Omega & \sum_{Y^{\frac{16}{135}}<p \leq(2 Y)^{\frac{1}{2}}} \sum_{Y^{\frac{16}{135}}<q<\min \left(p,\left(\frac{2 Y}{p}\right)^{\frac{1}{2}}\right)} S\left(\mathcal{A}_{p q}, q\right) \\
& \geq \sum_{i=1}^{69} \sum_{(p, q) \in D_{i}} S\left(\mathcal{A}_{p q}, q\right)=\sum_{i=1}^{69} \Omega_{i},
\end{aligned}
$$

where

$$
\begin{aligned}
& D_{1}=\left\{(p, q): Y^{\frac{16}{135}}<p \leq Y^{\frac{89}{462}}, Y^{\frac{16}{135}}<q<p\right\}, \\
& D_{2}=\left\{(p, q): Y^{\frac{89}{462}}<p \leq Y^{\frac{41}{180}}, Y^{\frac{16}{135}}<q<p^{-\frac{29}{48}} Y^{\frac{89}{288}}\right\}, \\
& D_{3}=\left\{(p, q): Y^{\frac{41}{180}}<p \leq Y^{\frac{8}{33}}, Y^{\frac{16}{135}}<q<p^{-\frac{29}{48}} Y^{\frac{89}{288}}\right\}, \\
& D_{4}=\left\{(p, q): Y^{\frac{41}{180}}<p \leq Y^{\frac{8}{33}}, p^{-1} Y^{\frac{41}{90}}<q<p\right\}, \\
& D_{5}=\left\{(p, q): Y^{\frac{8}{33}}<p \leq Y^{\frac{37}{150}}, Y^{\frac{16}{135}}<q<p^{-\frac{29}{48}} Y^{\frac{89}{288}}\right\}, \\
& D_{6}=\left\{(p, q): Y^{\frac{8}{33}}<p \leq Y^{\frac{37}{150}}, p^{-1} Y^{\frac{41}{90}}<q<p^{-1} Y^{\frac{16}{33}}\right\}, \\
& D_{7}=\left\{(p, q): Y^{\frac{37}{150}}<p \leq Y^{\frac{17}{63}}, Y^{\frac{16}{135}}<q<p^{-\frac{29}{48}} Y^{\frac{89}{288}}\right\}, \\
& D_{8}=\left\{(p, q): Y^{\frac{37}{150}}<p \leq Y^{\frac{17}{63}}, p^{-1} Y^{\frac{771}{1566}}<q<p^{-1} Y^{\frac{16}{33}}\right\}, \\
& D_{9}=\left\{(p, q): Y^{\frac{17}{63}}<p \leq Y^{\frac{17}{57}}, Y^{\frac{16}{135}}<q<p^{-\frac{29}{48}} Y^{\frac{89}{288}}\right\}, \\
& D_{10}=\left\{(p, q): Y^{\frac{17}{63}}<p \leq Y^{\frac{17}{57}}, p^{-1} Y^{\frac{701}{1566}}<q<p^{-1} Y^{\frac{16}{33}}\right\}, \\
& D_{11}=\left\{(p, q): Y^{\frac{17}{63}}<p \leq Y^{\frac{17}{57}}, p^{-\frac{6}{5}} Y^{\frac{17}{30}}<q<p^{-\frac{1}{8}} Y^{\frac{7}{24}}\right\}, \\
& D_{12}=\left\{(p, q): Y^{\frac{17}{57}}<p \leq Y^{\frac{31}{99}}, Y^{\frac{16}{135}}<q<p^{-\frac{29}{48}} Y^{\frac{89}{288}}\right\}, \\
& D_{13}=\left\{(p, q): Y^{\frac{17}{57}}<p \leq Y^{\frac{31}{99}}, p^{-1} Y^{\frac{701}{1566}}<q<p^{-1} Y^{\frac{16}{33}}\right\}, \\
& D_{14}=\left\{(p, q): Y^{\frac{17}{57}}<p \leq Y^{\frac{31}{99}}, p^{-\frac{12}{11}} Y^{\frac{17}{33}}<q<p^{-\frac{1}{8}} Y^{\frac{7}{24}}\right\},
\end{aligned}
$$




$$
\begin{aligned}
& D_{15}=\left\{(p, q): Y^{\frac{31}{99}}<p \leq Y^{\frac{17}{54}}, Y^{\frac{16}{135}}<q<p^{-\frac{29}{48}} Y^{\frac{89}{288}}\right\} \text {, } \\
& D_{16}=\left\{(p, q): Y^{\frac{31}{99}}<p \leq Y^{\frac{17}{54}}, p^{-1} Y^{\frac{701}{1566}}<q<p^{-\frac{58}{67}} Y^{\frac{89}{201}}\right\} \text {, } \\
& D_{17}=\left\{(p, q): Y^{\frac{31}{99}}<p \leq Y^{\frac{17}{54}}, p^{-\frac{12}{11}} Y^{\frac{17}{33}}<q<p^{-\frac{1}{8}} Y^{\frac{7}{24}}\right\} \text {, } \\
& D_{18}=\left\{(p, q): Y^{\frac{17}{54}}<p \leq Y^{\frac{221}{693}}, p^{-1} Y^{\frac{701}{1566}}<q<p^{-\frac{58}{67}} Y^{\frac{89}{201}}\right\} \text {, } \\
& D_{19}=\left\{(p, q): Y^{\frac{17}{54}}<p \leq Y^{\frac{221}{693}}, Y^{\frac{17}{99}}<q<p^{-\frac{1}{8}} Y^{\frac{7}{24}}\right\} \text {, } \\
& D_{20}=\left\{(p, q): Y^{\frac{221}{693}}<p \leq Y^{\frac{859}{2610}}, p^{-1} Y^{\frac{701}{1566}}<q<p^{-\frac{58}{67}} Y^{\frac{89}{201}}\right\} \text {, } \\
& D_{21}=\left\{(p, q): Y^{\frac{221}{693}}<p \leq Y^{\frac{859}{2610}}, Y^{\frac{17}{99}}<q<p^{-1} Y^{\frac{113}{198}}\right\} \text {, } \\
& D_{22}=\left\{(p, q): Y^{\frac{859}{2610}}<p \leq Y^{\frac{109}{330}}, Y^{\frac{16}{135}}<q<p^{-\frac{58}{67}} Y^{\frac{89}{201}}\right\} \text {, } \\
& D_{23}=\left\{(p, q): Y^{\frac{859}{2610}}<p \leq Y^{\frac{109}{330}}, Y^{\frac{17}{99}}<q<p^{-1} Y^{\frac{113}{198}}\right\} \text {, } \\
& D_{24}=\left\{(p, q): Y^{\frac{109}{330}}<p \leq Y^{\frac{151}{450}}, Y^{\frac{16}{135}}<q<p^{-\frac{58}{67}} Y^{\frac{89}{201}}\right\} \text {, } \\
& D_{25}=\left\{(p, q): Y^{\frac{109}{330}}<p \leq Y^{\frac{151}{450}}, Y^{\frac{17}{99}}<q<p^{-6} Y^{\frac{20}{9}}\right\} \text {, } \\
& D_{26}=\left\{(p, q): Y^{\frac{151}{450}}<p \leq Y^{\frac{34}{99}}, Y^{\frac{16}{135}}<q<p^{-\frac{58}{67}} Y^{\frac{89}{201}}\right\} \text {, } \\
& D_{27}=\left\{(p, q): Y^{\frac{151}{450}}<p \leq Y^{\frac{34}{99}}, Y^{\frac{17}{99}}<q<p^{\frac{1}{4}} Y^{\frac{1}{8}}\right\} \text {, } \\
& D_{28}=\left\{(p, q): Y^{\frac{151}{450}}<p \leq Y^{\frac{34}{99}}, p^{-1} Y^{\frac{5}{9}}<q<p^{\frac{1}{3}} Y^{\frac{7}{54}}\right\} \text {, } \\
& D_{29}=\left\{(p, q): Y^{\frac{34}{99}}<p \leq Y^{\frac{1649}{4752}}, Y^{\frac{16}{135}}<q<p^{-\frac{58}{67}} Y^{\frac{89}{201}}\right\} \text {, } \\
& D_{30}=\left\{(p, q): Y^{\frac{34}{99}}<p \leq Y^{\frac{1649}{4752}}, p^{-2} Y^{\frac{85}{99}}<q<p^{-\frac{58}{49}} Y^{\frac{85}{147}}\right\} \text {, } \\
& D_{31}=\left\{(p, q): Y^{\frac{34}{99}}<p \leq Y^{\frac{1649}{4752}}, p^{-\frac{58}{49}} Y^{\frac{85}{147}}<q<p^{\frac{1}{4}} Y^{\frac{1}{8}}\right\}, \\
& D_{32}=\left\{(p, q): Y^{\frac{1649}{4752}}<p \leq Y^{\frac{25}{72}}, Y^{\frac{16}{135}}<q<p^{-\frac{58}{67}} Y^{\frac{89}{201}}\right\} \text {, } \\
& D_{33}=\left\{(p, q): Y^{\frac{1649}{4752}}<p \leq Y^{\frac{25}{72}}, p^{-\frac{70}{59}} Y^{\frac{34}{59}}<q<p^{-\frac{58}{49}} Y^{\frac{85}{147}}\right\} \text {, } \\
& D_{34}=\left\{(p, q): Y^{\frac{1649}{4752}}<p \leq Y^{\frac{25}{72}}, p^{-\frac{58}{49}} Y^{\frac{85}{147}}<q<p^{\frac{1}{4}} Y^{\frac{1}{8}}\right\} \text {, } \\
& D_{35}=\left\{(p, q): Y^{\frac{25}{72}}<p \leq Y^{\frac{10}{27}}, Y^{\frac{16}{135}}<q<p^{-\frac{58}{67}} Y^{\frac{89}{201}}\right\} \text {, } \\
& D_{36}=\left\{(p, q): Y^{\frac{25}{72}}<p \leq Y^{\frac{10}{27}}, p^{-\frac{70}{59}} Y^{\frac{34}{59}}<q<p^{-\frac{58}{49}} Y^{\frac{85}{147}}\right\} \text {, } \\
& D_{37}=\left\{(p, q): Y^{\frac{25}{72}}<p \leq Y^{\frac{10}{27}}, p^{-\frac{58}{49}} Y^{\frac{85}{147}}<q<p^{-\frac{23}{15}} Y^{\frac{7}{9}}\right\} \text {, } \\
& D_{38}=\left\{(p, q): Y^{\frac{10}{27}}<p \leq Y^{\frac{617}{1620}}, p^{-\frac{70}{59}} Y^{\frac{34}{59}}<q<p^{-\frac{58}{49}} Y^{\frac{85}{147}}\right\} \text {, } \\
& D_{39}=\left\{(p, q): Y^{\frac{10}{27}}<p \leq Y^{\frac{617}{1620}}, p^{-\frac{58}{49}} Y^{\frac{85}{147}}<q<p^{-1} Y^{\frac{47}{81}}\right\} \text {, } \\
& D_{40}=\left\{(p, q): Y^{\frac{617}{1620}}<p \leq Y^{\frac{1823}{4725}}, p^{-\frac{70}{59}} Y^{\frac{34}{59}}<q<p^{-\frac{58}{49}} Y^{\frac{85}{147}}\right\} \text {, } \\
& D_{41}=\left\{(p, q): Y^{\frac{617}{1620}}<p \leq Y^{\frac{1823}{4725}}, p^{-\frac{58}{49}} Y^{\frac{85}{147}}<q<p^{-\frac{29}{19}} Y^{\frac{89}{114}}\right\} \text {, } \\
& D_{42}=\left\{(p, q): Y^{\frac{1823}{4725}}<p \leq Y^{\frac{3041}{7830}}, Y^{\frac{16}{135}}<q<p^{-\frac{58}{49}} Y^{\frac{85}{147}}\right\} \text {, } \\
& D_{43}=\left\{(p, q): Y^{\frac{1823}{4725}}<p \leq Y^{\frac{3041}{7830}}, p^{-\frac{58}{49}} Y^{\frac{85}{147}}<q<p^{-\frac{29}{19}} Y^{\frac{89}{114}}\right\} \text {, } \\
& D_{44}=\left\{(p, q): Y^{\frac{3041}{7830}}<p \leq Y^{\frac{11}{27}}, Y^{\frac{16}{135}}<q<p^{-\frac{29}{19}} Y^{\frac{89}{114}}\right\} \text {, } \\
& D_{45}=\left\{(p, q): Y^{\frac{11}{27}}<p \leq Y^{\frac{34}{81}}, Y^{\frac{16}{135}}<q<p^{-\frac{29}{19}} Y^{\frac{89}{114}}\right\}
\end{aligned}
$$




$$
\begin{aligned}
& D_{46}=\left\{(p, q): Y^{\frac{11}{27}}<p \leq Y^{\frac{34}{81}}, p^{-\frac{29}{19}} Y^{\frac{89}{114}}<q<p^{-\frac{35}{23}} Y^{\frac{18}{23}}\right\} \text {, } \\
& D_{47}=\left\{(p, q): Y^{\frac{11}{27}}<p \leq Y^{\frac{34}{81}}, p^{-\frac{23}{8}} Y^{\frac{17}{12}}<q<p^{-\frac{1}{4}} Y^{\frac{25}{72}}\right\} \text {, } \\
& D_{48}=\left\{(p, q): Y^{\frac{34}{81}}<p \leq Y^{\frac{49}{114}}, Y^{\frac{16}{135}}<q<p^{-\frac{29}{19}} Y^{\frac{89}{114}}\right\} \text {, } \\
& D_{49}=\left\{(p, q): Y^{\frac{34}{81}}<p \leq Y^{\frac{49}{114}}, p^{-\frac{29}{19}} Y^{\frac{89}{114}}<q<p^{-\frac{35}{23}} Y^{\frac{18}{23}}\right\} \text {, } \\
& D_{50}=\left\{(p, q): Y^{\frac{34}{81}}<p \leq Y^{\frac{49}{114}}, p^{-\frac{29}{10}} Y^{\frac{17}{12}}<q<p^{-\frac{1}{4}} Y^{\frac{25}{72}}\right\} \text {, } \\
& D_{51}=\left\{(p, q): Y^{\frac{49}{114}}<p \leq Y^{\frac{3397}{7830}}, Y^{\frac{16}{135}}<q<p^{-\frac{29}{19}} Y^{\frac{89}{114}}\right\} \text {, } \\
& D_{52}=\left\{(p, q): Y^{\frac{49}{114}}<p \leq Y^{\frac{3397}{7830}}, p^{-\frac{29}{10}} Y^{\frac{17}{12}}<q<p^{\frac{1}{7}} Y^{\frac{4}{21}}\right\}, \\
& D_{53}=\left\{(p, q): Y^{\frac{3397}{7830}}<p \leq Y^{\frac{4}{9}}, p^{-\frac{35}{12}} Y^{\frac{17}{12}}<q<p^{-\frac{29}{10}} Y^{\frac{17}{12}}\right\} \text {, } \\
& D_{54}=\left\{(p, q): Y^{\frac{3397}{7830}}<p \leq Y^{\frac{4}{9}}, p^{-\frac{29}{10}} Y^{\frac{17}{12}}<q<p^{\frac{1}{7}} Y^{\frac{4}{21}}\right\} \text {, } \\
& D_{55}=\left\{(p, q): Y^{\frac{4}{9}}<p \leq Y^{\frac{701}{1566}}, p^{-\frac{29}{10}} Y^{\frac{17}{12}}<q<p^{-\frac{1}{5}} Y^{\frac{3}{10}}\right\}, \\
& D_{56}=\left\{(p, q): Y^{\frac{4}{9}}<p \leq Y^{\frac{701}{1566}}, p^{-\frac{6}{5}} Y^{\frac{34}{45}}<q<p^{\frac{1}{7}} Y^{\frac{4}{21}}\right\} \text {, } \\
& D_{57}=\left\{(p, q): Y^{\frac{701}{1566}}<p \leq Y^{\frac{41}{90}}, Y^{\frac{16}{135}}<q<p^{-\frac{1}{5}} Y^{\frac{3}{10}}\right\} \text {, } \\
& D_{58}=\left\{(p, q): Y^{\frac{701}{1566}}<p \leq Y^{\frac{41}{90}}, p^{-\frac{6}{5}} Y^{\frac{34}{45}}<q<p^{\frac{1}{7}} Y^{\frac{4}{21}}\right\} \text {, } \\
& D_{59}=\left\{(p, q): Y^{\frac{41}{90}}<p \leq Y^{\frac{17}{36}}, Y^{\frac{16}{135}}<q<p^{\frac{1}{7}} Y^{\frac{4}{21}}\right\} \text {, } \\
& D_{60}=\left\{(p, q): Y^{\frac{17}{36}}<p \leq Y^{\frac{16}{33}}, Y^{\frac{16}{135}}<q<p^{-1} Y^{\frac{46}{63}}\right\} \text {, } \\
& D_{61}=\left\{(p, q): Y^{\frac{16}{33}}<p \leq Y^{\frac{307}{630}}, Y^{\frac{16}{135}}<q<p^{-\frac{58}{9}} Y^{\frac{89}{27}}\right\} \text {, } \\
& D_{62}=\left\{(p, q): Y^{\frac{16}{33}}<p \leq Y^{\frac{307}{630}}, Y^{\frac{17}{99}}<q<p^{-1} Y^{\frac{46}{63}}\right\} \text {, } \\
& D_{63}=\left\{(p, q): Y^{\frac{307}{630}}<p \leq Y^{\frac{211}{432}}, Y^{\frac{16}{135}}<q<p^{-\frac{58}{9}} Y^{\frac{89}{27}}\right\} \text {, } \\
& D_{64}=\left\{(p, q): Y^{\frac{307}{630}}<p \leq Y^{\frac{211}{432}}, Y^{\frac{17}{99}}<q<p^{-6} Y^{\frac{19}{6}}\right\} \text {, } \\
& D_{65}=\left\{(p, q): Y^{\frac{211}{432}}<p \leq Y^{\frac{281}{570}}, Y^{\frac{16}{135}}<q<p^{-\frac{58}{9}} Y^{\frac{89}{27}}\right\} \text {, } \\
& D_{66}=\left\{(p, q): Y^{\frac{211}{432}}<p \leq Y^{\frac{281}{570}}, p^{-\frac{58}{9}} Y^{\frac{89}{27}}<q<p^{-\frac{70}{11}} Y^{\frac{36}{11}}\right\} \text {, } \\
& D_{67}=\left\{(p, q): Y^{\frac{211}{432}}<p \leq Y^{\frac{281}{570}}, Y^{\frac{17}{99}}<q<p^{-6} Y^{\frac{19}{6}}\right\} \text {, } \\
& D_{68}=\left\{(p, q): Y^{\frac{281}{570}}<p \leq Y^{\frac{429}{870}}, Y^{\frac{16}{135}}<q<p^{-\frac{58}{9}} Y^{\frac{89}{27}}\right\} \text {, } \\
& D_{69}=\left\{(p, q): Y^{\frac{429}{870}}<p \leq Y^{\frac{1}{2}}, Y^{\frac{17}{99}}<q<p^{-1} Y^{\frac{40}{57}}\right\} \text {. }
\end{aligned}
$$

\section{Bounds of the sieve functions}

LEMMA 24. We have

$$
\begin{aligned}
\Omega_{11}+ & \Omega_{14}+\Omega_{17}+\Omega_{19}+\Omega_{21}+\Omega_{23}+\Omega_{25}+\Omega_{27}+\Omega_{28}+\Omega_{31} \\
& +\Omega_{34}+\Omega_{37}+\Omega_{39}+\Omega_{41}+\Omega_{43}+\Omega_{44}+\Omega_{45}+\Omega_{48}+\Omega_{51} \\
& \geq 0.422726 \cdot \frac{C(n) Y}{\log Y \log N} .
\end{aligned}
$$


Proof. We have

$$
\Omega_{11}=\sum_{Y^{\frac{17}{63}}<p \leq Y^{\frac{17}{57}}} \sum_{p^{-\frac{6}{5}} Y^{\frac{17}{30}}<q<p^{-\frac{1}{8}} Y^{\frac{7}{24}}} S\left(\mathcal{A}_{p q}, q\right)=\sum_{p q r+p_{1}=n} 1,
$$

where $p q r \leq 2 Y, N-Y<p_{1} \leq N, Y^{\frac{17}{63}}<p \leq Y^{\frac{17}{57}}, p^{-\frac{6}{5}} Y^{\frac{17}{30}}<q<p^{-\frac{1}{8}} Y^{\frac{7}{24}}$, and the least prime factor of $r$ is greater than $q$.

Let $h=q$ and $k=r$. By Lemma 8 with region (i), (8) holds. Then Lemmas 18 and 21 yield

$$
\begin{aligned}
& \Omega_{11}=\frac{C(n)}{\log N} \sum_{Y^{\frac{17}{63}}<p \leq Y^{\frac{17}{57}}} \sum_{p^{-\frac{6}{5}} \sum_{Y^{\frac{17}{30}}<q<p^{-\frac{1}{8}}}} S\left(\mathcal{B}_{p q}^{\frac{7}{24}}, q\right)+O\left(\frac{\varepsilon C(n) Y}{\log ^{2} N}\right)
\end{aligned}
$$

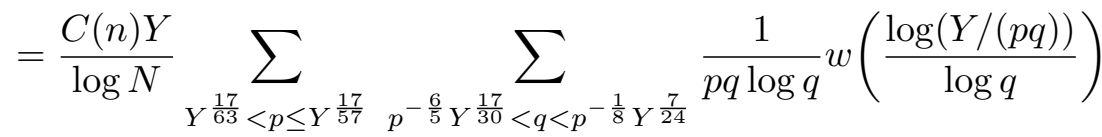

$$
\begin{aligned}
& +O\left(\frac{\varepsilon C(n) Y}{\log ^{2} N}\right) \\
& =\frac{C(n) Y}{\log Y \log N} \int_{\frac{17}{63}}^{\frac{17}{57}} \frac{d t}{t} \int_{\frac{17}{30}-\frac{6}{5} t}^{\frac{7}{24}-\frac{t}{8}} \frac{1}{u^{2}} w\left(\frac{1-t-u}{u}\right) d u+O\left(\frac{\varepsilon C(n) Y}{\log ^{2} N}\right) \\
& =\frac{C(n) Y}{\log Y \log N}\left(\int_{\frac{17}{63}}^{\frac{17}{57}} \frac{d t}{t} \int_{\frac{17}{30}-\frac{6}{5} t}^{\frac{1}{3}(1-t)} \frac{1}{u(1-t-u)}\left(1+\log \left(\frac{1-t}{u}-2\right)\right) d u\right. \\
& \left.+\int_{\frac{17}{63}}^{\frac{17}{57}} \frac{d t}{t} \int_{\frac{1}{3}(1-t)}^{\frac{7}{24}-\frac{t}{8}} \frac{d u}{u(1-t-u)}+O(\varepsilon)\right) \\
& \geq(0.012655+0.015106) \cdot \frac{C(n) Y}{\log Y \log N} \\
& =0.027761 \cdot \frac{C(n) Y}{\log Y \log N} .
\end{aligned}
$$

Using the above discussion and Lemma 20, we have

$$
\begin{gathered}
\Omega_{14}+\Omega_{17}+\Omega_{19}+\Omega_{21}+\Omega_{23}+\Omega_{25}+\Omega_{27}+\Omega_{28}+\Omega_{31} \\
+\Omega_{34}+\Omega_{37}+\Omega_{39}+\Omega_{41}+\Omega_{43}+\Omega_{44}+\Omega_{45}+\Omega_{48}+\Omega_{51} \\
=\frac{C(n) Y}{\log Y \log N}\left(\int_{\frac{17}{57}}^{\frac{17}{54}} \frac{d t}{t} \int_{\frac{17}{33}-\frac{12}{11} t}^{\frac{7}{24}-\frac{t}{8}} \frac{1}{u^{2}} w\left(\frac{1-t-u}{u}\right) d u\right. \\
\quad+\int_{\frac{17}{54}}^{\frac{221}{693}} \frac{d t}{t} \int_{\frac{17}{99}}^{\frac{7}{24}-\frac{t}{8}} \frac{1}{u^{2}} w\left(\frac{1-t-u}{u}\right) d u+\int_{\frac{221}{693}}^{\frac{109}{330}} \frac{d t}{t} \int_{\frac{17}{99}}^{\frac{113}{198}-t} \frac{1}{u^{2}} w\left(\frac{1-t-u}{u}\right) d u
\end{gathered}
$$




$$
\begin{aligned}
& +\int_{\frac{109}{330}}^{\frac{151}{450}} \frac{d t}{t} \int_{\frac{17}{99}}^{\frac{20}{9}-6 t} \frac{1}{u^{2}} w\left(\frac{1-t-u}{u}\right) d u+\int_{\frac{151}{450}}^{\frac{34}{99}} \frac{d t}{t} \int_{\frac{17}{99}}^{\frac{1}{8}+\frac{t}{4}} \frac{1}{u^{2}} w\left(\frac{1-t-u}{u}\right) d u \\
& +\int_{\frac{151}{450}}^{\frac{34}{99}} \frac{d t}{t} \int_{\frac{5}{9}-t}^{\frac{7}{54}+\frac{t}{3}} \frac{1}{u^{2}} w\left(\frac{1-t-u}{u}\right) d u+\int_{\frac{34}{99}}^{\frac{25}{72}} \frac{d t}{t} \int_{\frac{85}{147}-\frac{58}{49} t}^{\frac{1}{8}+\frac{t}{4}} \frac{1}{u^{2}} w\left(\frac{1-t-u}{u}\right) d u \\
& +\int_{\frac{25}{72}}^{\frac{10}{27}} \frac{d t}{t} \int_{\frac{85}{147}-\frac{58}{49} t}^{\frac{7}{9}-\frac{23}{15} t} \frac{1}{u^{2}} w\left(\frac{1-t-u}{u}\right) d u+\int_{\frac{10}{27}}^{\frac{617}{1620}} \frac{d t}{t} \int_{\frac{85}{147}-\frac{58}{49} t}^{\frac{47}{81}-t} \frac{1}{u^{2}} w\left(\frac{1-t-u}{u}\right) d u \\
& +\int_{\frac{617}{1620}}^{\frac{3041}{7830}} \frac{d t}{t} \int_{\frac{85}{147}-\frac{58}{49} t}^{\frac{89}{114}-\frac{29}{19} t} \frac{1}{u^{2}} w\left(\frac{1-t-u}{u}\right) d u \\
& \left.+\int_{\frac{3041}{7830}}^{\frac{3397}{7830}} \frac{d t}{t} \int_{\frac{16}{135}}^{\frac{89}{114}-\frac{29}{19} t} \frac{1}{u^{2}} w\left(\frac{1-t-u}{u}\right) d u+O(\varepsilon)\right) \\
& \geq \frac{C(n) Y}{\log Y \log N}\left(\int_{\frac{17}{57}}^{\frac{17}{54}} \frac{d t}{t} \int_{\frac{17}{33}-\frac{12}{11} t}^{\frac{1}{3}(1-t)} \frac{1}{u(1-t-u)}\left(1+\log \left(\frac{1-t}{u}-2\right)\right) d u\right. \\
& +\int_{\frac{17}{57}}^{\frac{17}{54}} \frac{d t}{t} \int_{\frac{1}{3}(1-t)}^{\frac{7}{24}-\frac{t}{8}} \frac{d u}{u(1-t-u)} \\
& +\int_{\frac{17}{54}}^{\frac{221}{693}} \frac{d t}{t} \int_{\frac{17}{99}}^{\frac{1}{3}(1-t)} \frac{1}{u(1-t-u)}\left(1+\log \left(\frac{1-t}{u}-2\right)\right) d u \\
& +\int_{\frac{17}{54}}^{\frac{221}{693}} \frac{d t}{t} \int_{\frac{1}{3}(1-t)}^{\frac{7}{24}-\frac{t}{8}} \frac{d u}{u(1-t-u)} \\
& +\int_{\frac{221}{693}}^{\frac{109}{330}} \frac{d t}{t} \int_{\frac{17}{99}}^{\frac{1}{3}(1-t)} \frac{1}{u(1-t-u)}\left(1+\log \left(\frac{1-t}{u}-2\right)\right) d u \\
& +\int_{\frac{221}{693}}^{\frac{109}{330}} \frac{d t}{t} \int_{\frac{1}{3}(1-t)}^{\frac{113}{198}-t} \frac{d u}{u(1-t-u)} \\
& +\int_{\frac{109}{330}}^{\frac{1}{3}} \frac{d t}{t} \int_{\frac{17}{99}}^{\frac{1}{3}(1-t)} \frac{1}{u(1-t-u)}\left(1+\log \left(\frac{1-t}{u}-2\right)\right) d u
\end{aligned}
$$




$$
\begin{aligned}
& +\int_{\frac{109}{330}}^{\frac{1}{3}} \frac{d t}{t} \int_{\frac{1}{3}(1-t)}^{\frac{20}{9}-6 t} \frac{d u}{u(1-t-u)} \\
& +\int_{\frac{1}{3}}^{\frac{151}{450}} \frac{d t}{t} \int_{\frac{17}{99}}^{\frac{20}{9}-6 t} \frac{1}{u(1-t-u)}\left(1+\log \left(\frac{1-t}{u}-2\right)\right) d u \\
& +\int_{\frac{151}{450}}^{\frac{34}{99}} \frac{d t}{t} \int_{\frac{17}{99}}^{\frac{1}{8}+\frac{t}{4}} \frac{1}{u(1-t-u)}\left(1+\log \left(\frac{1-t}{u}-2\right)\right) d u \\
& +\int_{\frac{151}{450}}^{\frac{34}{99}} \frac{d t}{t} \int_{\frac{5}{9}-t}^{\frac{1}{3}(1-t)} \frac{1}{u(1-t-u)}\left(1+\log \left(\frac{1-t}{u}-2\right)\right) d u \\
& +\int_{\frac{151}{450}}^{\frac{34}{99}} \frac{d t}{t} \int_{\frac{1}{3}(1-t)}^{\frac{7}{54}+\frac{t}{3}} \frac{d u}{u(1-t-u)} \\
& +\int_{\frac{34}{99}}^{\frac{25}{72}} \frac{d t}{t} \int_{\frac{85}{147}-\frac{58}{49} t}^{\frac{1}{8}+\frac{t}{4}} \frac{1}{u(1-t-u)}\left(1+\log \left(\frac{1-t}{u}-2\right)\right) d u \\
& +\int_{\frac{25}{72}}^{\frac{193}{549}} \frac{d t}{t} \int_{\frac{85}{147}-\frac{58}{49} t}^{\frac{1}{3}(1-t)} \frac{1}{u(1-t-u)}\left(1+\log \left(\frac{1-t}{u}-2\right)\right) d u \\
& +\int_{\frac{25}{72}}^{\frac{193}{549}} \frac{d t}{t} \int_{\frac{1}{3}(1-t)}^{\frac{7}{9}-\frac{23}{15} t} \frac{d u}{u(1-t-u)} \\
& +0.5607 \int_{\frac{193}{549}}^{\frac{10}{27}} \frac{d t}{t} \int_{\frac{85}{147}-\frac{58}{49} t}^{\frac{1}{4}(1-t)} \frac{d u}{u^{2}} \\
& +\int_{\frac{193}{549}}^{\frac{10}{27}} \frac{d t}{t} \int_{\frac{1}{4}(1-t)}^{\frac{1}{3}(1-t)} \frac{1}{u(1-t-u)}\left(1+\log \left(\frac{1-t}{u}-2\right)\right) d u \\
& +\int_{\frac{193}{549}}^{\frac{10}{27}} \frac{d t}{t} \int_{\frac{1}{3}(1-t)}^{\frac{7}{9}-\frac{23}{15} t} \frac{d u}{u(1-t-u)} \\
& +0.5607 \int_{\frac{10}{27}}^{\frac{617}{1620}} \frac{d t}{t} \int_{\frac{85}{147}-\frac{58}{49} t}^{\frac{1}{4}(1-t)} \frac{d u}{u^{2}}
\end{aligned}
$$




$$
\begin{aligned}
& +\int_{\frac{10}{27}}^{\frac{617}{1620}} \frac{d t}{t} \int_{\frac{1}{4}(1-t)}^{\frac{47}{81}-t} \frac{1}{u(1-t-u)}\left(1+\log \left(\frac{1-t}{u}-2\right)\right) d u \\
& +0.5607 \int_{\frac{617}{1620}}^{\frac{3041}{7830}} \frac{d t}{t} \int_{\frac{85}{147}-\frac{58}{49} t}^{\frac{1}{4}(1-t)} \frac{d u}{u^{2}} \\
& +\int_{\frac{617}{1620}}^{\frac{3041}{7830}} \frac{d t}{t} \int_{\frac{1}{4}(1-t)}^{\frac{89}{114}-\frac{29}{19} t} \frac{1}{u(1-t-u)}\left(1+\log \left(\frac{1-t}{u}-2\right)\right) d u \\
& +0.5607 \int_{\frac{3041}{7830}}^{\frac{121}{291}} \frac{d t}{t} \int_{\frac{16}{135}}^{\frac{1}{4}(1-t)} \frac{d u}{u^{2}} \\
& +\int_{\frac{3041}{7830}}^{\frac{121}{291}} \frac{d t}{t} \int_{\frac{1}{4}(1-t)}^{\frac{89}{114}-\frac{29}{19} t} \frac{1}{u(1-t-u)}\left(1+\log \left(\frac{1-t}{u}-2\right)\right) d u \\
& \left.+0.5607 \int_{\frac{121}{291}}^{\frac{3397}{7830}} \frac{d t}{t} \int_{\frac{16}{135}}^{\frac{89}{114}-\frac{29}{19} t} \frac{d u}{u^{2}}+O(\varepsilon)\right) \\
& \geq(0.035949+0.010981+0.010207+0.002956 \\
& +0.026780+0.007110+0.006712+0.000780 \\
& +0.004363+0.013697+0.001020+0.005290 \\
& +0.007124+0.009992+0.003228+0.010892 \\
& +0.045105+0.006377+0.016938+0.023556 \\
& +0.017909+0.014602+0.066888+0.026127 \\
& +0.020382) \cdot \frac{C(n) Y}{\log Y \log N} \\
& =0.394965 \cdot \frac{C(n) Y}{\log Y \log N} \text {. }
\end{aligned}
$$

The proof of Lemma 24 is complete.

LEMma 25. We have

$$
\begin{aligned}
\Phi= & \Omega_{47}+\Omega_{50}+\Omega_{52}+\Omega_{54}+\Omega_{55}+\Omega_{56}+\Omega_{57}+\Omega_{58}+\Omega_{59} \\
& +\Omega_{60}+\Omega_{61}+\Omega_{62}+\Omega_{63}+\Omega_{64}+\Omega_{65}+\Omega_{67}+\Omega_{68}+\Omega_{69} \\
\geq & 0.347181 \cdot \frac{C(n) Y}{\log Y \log N} .
\end{aligned}
$$


Pr o of. On applying Lemmas 10, 18, 20 and 21, in the same way as in Lemma 24, we have

$$
\begin{aligned}
& \Phi=\frac{C(n) Y}{\log Y \log N}\left(\int_{\frac{11}{27}}^{\frac{34}{81}} \frac{d t}{t} \int_{\frac{17}{12}-\frac{23}{8} t}^{\frac{25}{72}-\frac{t}{4}} \frac{1}{u^{2}} w\left(\frac{1-t-u}{u}\right) d u\right. \\
& +\int_{\frac{34}{81}}^{\frac{49}{114}} \frac{d t}{t} \int_{\frac{17}{12}-\frac{29}{10} t}^{\frac{25}{72}-\frac{t}{4}} \frac{1}{u^{2}} w\left(\frac{1-t-u}{u}\right) d u \\
& +\int_{\frac{49}{114}}^{\frac{4}{9}} \frac{d t}{t} \int_{\frac{17}{12}-\frac{29}{10} t}^{\frac{4}{21}+\frac{t}{7}} \frac{1}{u^{2}} w\left(\frac{1-t-u}{u}\right) d u \\
& +\int_{\frac{4}{9}}^{\frac{701}{1566}} \frac{d t}{t} \int_{\frac{17}{12}-\frac{29}{10} t}^{\frac{3}{10}-\frac{t}{5}} \frac{1}{u^{2}} w\left(\frac{1-t-u}{u}\right) d u \\
& +\int_{\frac{4}{9}}^{\frac{701}{1566}} \frac{d t}{t} \int_{\frac{34}{45}-\frac{6}{5} t}^{\frac{4}{21}+\frac{t}{7}} \frac{1}{u^{2}} w\left(\frac{1-t-u}{u}\right) d u \\
& +\int_{\frac{701}{1566}}^{\frac{41}{90}} \frac{d t}{t} \int_{\frac{16}{135}}^{\frac{3}{10}-\frac{t}{5}} \frac{1}{u^{2}} w\left(\frac{1-t-u}{u}\right) d u \\
& +\int_{\frac{701}{1566}}^{\frac{41}{90}} \frac{d t}{t} \int_{\frac{34}{45}-\frac{6}{5} t}^{\frac{4}{21}+\frac{t}{7}} \frac{1}{u^{2}} w\left(\frac{1-t-u}{u}\right) d u \\
& +\int_{\frac{41}{90}}^{\frac{17}{36}} \frac{d t}{t} \int_{\frac{16}{135}}^{\frac{4}{21}+\frac{t}{7}} \frac{1}{u^{2}} w\left(\frac{1-t-u}{u}\right) d u \\
& +\int_{\frac{17}{36}}^{\frac{16}{33}} \frac{d t}{t} \int_{\frac{16}{135}}^{\frac{46}{63}-t} \frac{1}{u^{2}} w\left(\frac{1-t-u}{u}\right) d u+\int_{\frac{16}{33}}^{\frac{307}{630}} \frac{d t}{t} \int_{\frac{16}{135}}^{\frac{89}{27}-\frac{58}{9} t} \frac{1}{u^{2}} w\left(\frac{1-t-u}{u}\right) d u \\
& +\int_{\frac{16}{33}}^{\frac{307}{630}} \frac{d t}{t} \int_{\frac{17}{99}}^{\frac{46}{63}-t} \frac{1}{u^{2}} w\left(\frac{1-t-u}{u}\right) d u+\int_{\frac{307}{630}}^{\frac{281}{570}} \frac{d t}{t} \int_{\frac{16}{135}}^{\frac{89}{27}-\frac{58}{9} t} \frac{1}{u^{2}} w\left(\frac{1-t-u}{u}\right) d u \\
& +\int_{\frac{307}{630}}^{\frac{281}{570}} \frac{d t}{t} \int_{\frac{17}{99}}^{\frac{19}{6}-6 t} \frac{1}{u^{2}} w\left(\frac{1-t-u}{u}\right) d u
\end{aligned}
$$




$$
\begin{aligned}
& +\int_{\frac{281}{570}}^{\frac{429}{870}} \frac{d t}{t} \int_{\frac{16}{135}}^{\frac{89}{27}-\frac{58}{9} t} \frac{1}{u^{2}} w\left(\frac{1-t-u}{u}\right) d u \\
& \left.+\int_{\frac{429}{870}}^{\frac{1}{2}} \frac{d t}{t} \int_{\frac{17}{99}}^{\frac{40}{57}-t} \frac{1}{u^{2}} w\left(\frac{1-t-u}{u}\right) d u+O(\varepsilon)\right) \\
& \geq \frac{C(n) Y}{\log Y \log N}\left(\int_{\frac{11}{27}}^{\frac{34}{81}} \frac{d t}{t} \int_{\frac{17}{12}-\frac{23}{8} t}^{\frac{25}{72}-\frac{t}{4}} \frac{d u}{u(1-t-u)}\right. \\
& +\int_{\frac{34}{81}}^{\frac{65}{154}} \frac{d t}{t} \int_{\frac{17}{12}-\frac{29}{10} t}^{\frac{25}{72}-\frac{t}{4}} \frac{d u}{u(1-t-u)} \\
& +\int_{\frac{65}{154}}^{\frac{49}{114}} \frac{d t}{t} \int_{\frac{17}{12}-\frac{29}{10} t}^{\frac{1}{3}(1-t)} \frac{1}{u(1-t-u)}\left(1+\log \left(\frac{1-t}{u}-2\right)\right) d u \\
& +\int_{\frac{65}{154}}^{\frac{49}{114}} \frac{d t}{t} \int_{\frac{1}{3}(1-t)}^{\frac{25}{72}-\frac{t}{4}} \frac{d u}{u(1-t-u)} \\
& +\int_{\frac{49}{114}}^{\frac{70}{159}} \frac{d t}{t} \int_{\frac{17}{12}-\frac{29}{10} t}^{\frac{1}{3}(1-t)} \frac{1}{u(1-t-u)}\left(1+\log \left(\frac{1-t}{u}-2\right)\right) d u \\
& +\int_{\frac{49}{114}}^{\frac{70}{159}} \frac{d t}{t} \int_{\frac{1}{3}(1-t)}^{\frac{4}{21}+\frac{t}{7}} \frac{d u}{u(1-t-u)} \\
& +0.5607 \int_{\frac{70}{159}}^{\frac{4}{9}} \frac{d t}{t} \int_{\frac{17}{12}-\frac{29}{10} t}^{\frac{1}{4}(1-t)} \frac{d u}{u^{2}} \\
& +\int_{\frac{70}{159}}^{\frac{4}{9}} \frac{d t}{t} \int_{\frac{1}{4}(1-t)}^{\frac{1}{3}(1-t)} \frac{1}{u(1-t-u)}\left(1+\log \left(\frac{1-t}{u}-2\right)\right) d u \\
& +\int_{\frac{70}{159}}^{\frac{4}{9}} \frac{d t}{t} \int_{\frac{1}{3}(1-t)}^{\frac{4}{21}+\frac{t}{7}} \frac{d u}{u(1-t-u)}+0.5607 \int_{\frac{4}{9}}^{\frac{701}{1566}} \frac{d t}{t} \int_{\frac{17}{12}-\frac{29}{10} t}^{\frac{1}{4}(1-t)} \frac{d u}{u^{2}} \\
& +\int_{\frac{4}{9}}^{\frac{701}{1566}} \frac{d t}{t} \int_{\frac{1}{4}(1-t)}^{\frac{1}{3}(1-t)} \frac{1}{u(1-t-u)}\left(1+\log \left(\frac{1-t}{u}-2\right)\right) d u
\end{aligned}
$$




$$
\begin{aligned}
& +\int_{\frac{4}{9}}^{\frac{701}{1566}} \frac{d t}{t} \int_{\frac{1}{3}(1-t)}^{\frac{3}{10}-\frac{t}{5}} \frac{d u}{u(1-t-u)}+\int_{\frac{4}{9}}^{\frac{701}{1566}} \frac{d t}{t} \int_{\frac{34}{45}-\frac{6}{5} t}^{\frac{4}{21}+\frac{t}{7}} \frac{d u}{u(1-t-u)} \\
& +0.5607 \int_{\frac{701}{1566}}^{\frac{41}{90}} \frac{d t}{t} \int_{\frac{16}{135}}^{\frac{1}{4}(1-t)} \frac{d u}{u^{2}} \\
& +\int_{\frac{701}{1566}}^{\frac{41}{90}} \frac{d t}{t} \int_{\frac{1}{4}(1-t)}^{\frac{1}{3}(1-t)} \frac{1}{u(1-t-u)}\left(1+\log \left(\frac{1-t}{u}-2\right)\right) d u \\
& +\int_{\frac{701}{1566}}^{\frac{41}{90}} \frac{d t}{t} \int_{\frac{1}{3}(1-t)}^{\frac{3}{10}-\frac{t}{5}} \frac{d u}{u(1-t-u)}+\int_{\frac{701}{1566}}^{\frac{41}{90}} \frac{d t}{t} \int_{\frac{34}{45}-\frac{6}{5} t}^{\frac{4}{21}+\frac{t}{7}} \frac{d u}{u(1-t-u)} \\
& +0.5607 \int_{\frac{41}{90}}^{\frac{17}{36}} \frac{d t}{t} \int_{\frac{16}{135}}^{\frac{1}{4}(1-t)} \frac{d u}{u^{2}} \\
& +\int_{\frac{41}{90}}^{\frac{17}{36}} \frac{d t}{t} \int_{\frac{1}{4}(1-t)}^{\frac{1}{3}(1-t)} \frac{1}{u(1-t-u)}\left(1+\log \left(\frac{1-t}{u}-2\right)\right) d u \\
& +\int_{\frac{41}{90}}^{\frac{17}{36}} \frac{d t}{t} \int_{\frac{1}{3}(1-t)}^{\frac{4}{21}+\frac{t}{7}} \frac{d u}{u(1-t-u)}+0.5607 \int_{\frac{17}{36}}^{\frac{16}{33}} \frac{d t}{t} \int_{\frac{16}{135}}^{\frac{1}{4}(1-t)} \frac{d u}{u^{2}} \\
& +\int_{\frac{17}{36}}^{\frac{16}{33}} \frac{d t}{t} \int_{\frac{1}{4}(1-t)}^{\frac{1}{3}(1-t)} \frac{1}{u(1-t-u)}\left(1+\log \left(\frac{1-t}{u}-2\right)\right) d u \\
& +\int_{\frac{17}{36}}^{\frac{16}{33}} \frac{d t}{t} \int_{\frac{1}{3}(1-t)}^{\frac{46}{63}-t} \frac{d u}{u(1-t-u)}+0.5607 \int_{\frac{16}{33}}^{\frac{307}{630}} \frac{d t}{t} \int_{\frac{16}{135}}^{\frac{1}{4}(1-t)} \frac{d u}{u^{2}} \\
& +\int_{\frac{16}{33}}^{\frac{307}{630}} \frac{d t}{t} \int_{\frac{1}{4}(1-t)}^{\frac{89}{27}-\frac{58}{9} t} \frac{1}{u(1-t-u)}\left(1+\log \left(\frac{1-t}{u}-2\right)\right) d u \\
& +\int_{\frac{16}{33}}^{\frac{307}{630}} \frac{d t}{t} \int_{\frac{17}{99}}^{\frac{46}{63}-t} \frac{d u}{u(1-t-u)}+0.5607 \int_{\frac{307}{630}}^{\frac{329}{669}} \frac{d t}{t} \int_{\frac{16}{135}}^{\frac{1}{4}(1-t)} \frac{d u}{u^{2}} \\
& +\int_{\frac{307}{630}}^{\frac{329}{669}} \frac{d t}{t} \int_{\frac{1}{4}(1-t)}^{\frac{89}{27}-\frac{58}{9} t} \frac{1}{u(1-t-u)}\left(1+\log \left(\frac{1-t}{u}-2\right)\right) d u
\end{aligned}
$$




$$
\begin{aligned}
& +0.5607 \int_{\frac{329}{669}}^{\frac{281}{570}} \frac{d t}{t} \int_{\frac{16}{135}}^{\frac{89}{27}-\frac{58}{9} t} \frac{d u}{u^{2}}+\int_{\frac{307}{630}}^{\frac{281}{570}} \frac{d t}{t} \int_{\frac{17}{99}}^{\frac{19}{6}-6 t} \frac{d u}{u(1-t-u)} \\
& \left.+0.5607 \int_{\frac{281}{570}}^{\frac{429}{870}} \frac{d t}{t} \int_{\frac{16}{135}}^{\frac{89}{27}-\frac{58}{9} t} \frac{d u}{u^{2}}+\int_{\frac{429}{870}}^{\frac{1}{2}} \frac{d t}{t} \int_{\frac{17}{99}}^{\frac{40}{57}-t} \frac{d u}{u(1-t-u)}+O(\varepsilon)\right) \\
& \geq(0.005888+0.003229+0.002778+0.011651 \\
& +0.015140+0.020407+0.001606+0.009394 \\
& +0.008706+0.003624+0.007141+0.002659 \\
& +0.003233+0.011243+0.017671+0.006828 \\
& +0.009875+0.019672+0.037042+0.040808 \\
& +0.011345+0.027966+0.031531+0.001851 \\
& +0.004715+0.005786+0.003084+0.003848 \\
& +0.000433+0.010176+0.000003+0.007848) \cdot \frac{C(n) Y}{\log Y \log N} \\
& =0.347181 \cdot \frac{C(n) Y}{\log Y \log N} .
\end{aligned}
$$

The proof of Lemma 25 is complete.

Lemma 26. We have

$$
\begin{aligned}
\Omega_{4}+\Omega_{6}+\Omega_{8}+ & \Omega_{10}+\Omega_{13}+\Omega_{16}+\Omega_{18}+\Omega_{20} \\
& +\Omega_{22}+\Omega_{24}+\Omega_{26}+\Omega_{29}+\Omega_{32}+\Omega_{35} \\
\geq & 0.298946 \cdot \frac{C(n) Y}{\log Y \log N} .
\end{aligned}
$$

Proof. We have

$$
\begin{aligned}
\Omega_{4}= & \sum_{Y^{\frac{41}{180}<p \leq Y^{\frac{8}{33}}}} \sum_{p^{-1} Y^{\frac{41}{90}}<q<p} S\left(\mathcal{A}_{p q}, q\right) \\
= & \sum_{Y^{\frac{41}{180}<p \leq Y^{\frac{8}{33}}}} \sum_{p^{-1} Y^{\frac{41}{90}}<q<p} S\left(\mathcal{A}_{p q}, Y^{\delta}\right) \\
& -\sum_{Y^{\frac{41}{180}<p \leq Y^{33}}} \sum_{p^{-1} Y^{\frac{41}{90}}<q<p} \sum_{Y^{\delta}<r<Y^{\frac{16}{135}}} S\left(\mathcal{A}_{p q r}, r\right) \\
& -\sum_{Y^{\frac{41}{180}}<p \leq Y^{\frac{8}{33}}} \sum_{p^{-1} Y^{\frac{41}{90}}<q<p} S\left(\mathcal{Y}^{\frac{16}{135}}<r<\min \left(q,\left(\frac{2 Y}{p q}\right)^{\frac{1}{2}}\right)\right. \\
= & \Phi_{1}-\Phi_{2}-\Phi_{3} .
\end{aligned}
$$


Let $z=Y^{\delta}$ and $D=D(p, q)=Y^{\frac{19}{36}} /(p q)$. Applying Iwaniec's sieve method, we have

$$
\begin{aligned}
\Phi_{1} \leq & \frac{C(n) Y}{\log z \log N} \sum_{Y^{\frac{41}{180}<p \leq Y^{\frac{8}{33}}}} \sum_{p^{-1} Y^{\frac{41}{90}}<q<p} \frac{1}{p q} F\left(\frac{\log D}{\log z}\right) \\
& +\sum_{Y^{\frac{41}{180}<p \leq Y^{\frac{8}{33}}}} \sum_{p^{-1} Y^{\frac{41}{90}}<q<p} \sum_{r<Y^{\frac{19}{36}} /(p q)} a(r) \widetilde{r}(\mathcal{A}, p q r)+O\left(\frac{\delta C(n) Y}{\log ^{2} N}\right) .
\end{aligned}
$$

Let $m=p q r$. Then Lemma 17 yields

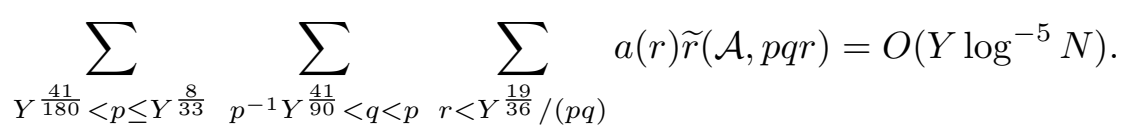

Hence,

$$
\begin{aligned}
\Phi_{1} & \leq \frac{e^{-\gamma}}{\delta} \cdot \frac{C(n) Y}{\log Y \log N} \sum_{Y^{\frac{41}{180}<p \leq Y^{\frac{8}{33}}}} \sum_{p^{-1} Y^{\frac{41}{90}}<q<p} \frac{1}{p q}+O\left(\frac{\delta C(n) Y}{\log ^{2} N}\right) \\
& =\frac{C(n)}{\log N} \sum_{Y^{\frac{41}{180}<p \leq Y^{\frac{8}{33}}}} \sum_{p^{-1} Y^{\frac{41}{90}}<q<p} S\left(\mathcal{B}_{p q}, Y^{\delta}\right)+O\left(\frac{\delta C(n) Y}{\log ^{2} N}\right) .
\end{aligned}
$$

In the same way, we can get the lower bound

$$
\Phi_{1} \geq \frac{C(n)}{\log N} \sum_{Y^{\frac{41}{180}}<p \leq Y^{\frac{8}{33}}} \sum_{p^{-1} Y^{\frac{41}{90}}<q<p} S\left(\mathcal{B}_{p q}, Y^{\delta}\right)+O\left(\frac{\delta C(n) Y}{\log ^{2} N}\right) .
$$

Thus we have the asymptotic formula

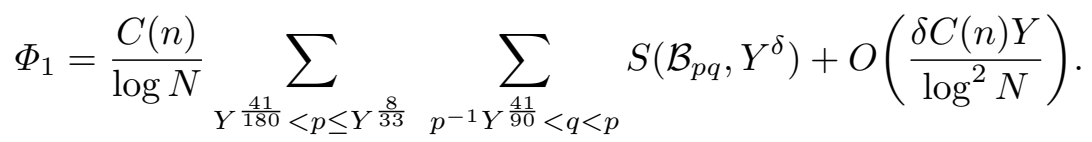

Using Lemma 19, we have

$$
\begin{aligned}
\Phi_{2}= & \frac{C(n)}{\log N} \sum_{Y^{\frac{41}{180}<p \leq Y^{\frac{8}{33}}}} \sum_{p^{-1} Y^{\frac{41}{90}}<q<p} \sum_{Y^{\delta}<r<Y^{\frac{16}{135}}} S\left(\mathcal{B}_{p q r}, r\right) \\
& +O\left(\frac{\delta C(n) Y}{\log ^{2} N}\right) .
\end{aligned}
$$

By Lemma 13 with region (i), (8) holds. Then Lemma 18 yields

$$
\begin{aligned}
\Phi_{3}= & \frac{C(n)}{\log N} \sum_{Y^{\frac{41}{180}<p \leq Y}} \sum_{p^{\frac{8}{33}}} \sum_{p^{-1} Y^{\frac{41}{90}}<q<p} \sum_{Y^{\frac{16}{135}}<r<\min \left(q,\left(\frac{2 Y}{p q}\right)^{\frac{1}{2}}\right)} S\left(\mathcal{B}_{p q r}, r\right) \\
& +O\left(\frac{\delta C(n) Y}{\log ^{2} N}\right) .
\end{aligned}
$$


Gathering together (53)-(56), we have

$$
\begin{aligned}
\Omega_{4}= & \frac{C(n)}{\log N} \sum_{Y^{\frac{41}{180}}<p \leq Y} \sum_{Y^{\frac{8}{33}}} S\left(\mathcal{B}_{p q}, q\right)+O\left(\frac{\delta C(n) Y}{\log ^{2} N}\right) \\
= & \frac{C(n) Y}{\log Y \log N} \int_{\frac{41}{180}}^{\frac{8}{33}} \frac{d t}{t} \int_{\frac{41}{90}-t}^{t} \frac{1}{u^{2}} w\left(\frac{1-t-u}{u}\right) d u+O\left(\frac{\delta C(n) Y}{\log ^{2} N}\right) \\
= & \frac{C(n) Y}{\log Y \log N} \int_{\frac{41}{180}}^{\frac{8}{33}} \frac{d t}{t} \int_{\frac{41}{90}-t}^{t} \frac{1}{u(1-t-u)}\left(1+\log \left(\frac{1-t}{u}-2\right)\right) d u \\
& +O\left(\frac{\delta C(n) Y}{\log ^{2} N}\right) \\
\geq & 0.009636 \cdot \frac{C(n)}{\log Y \log N} .
\end{aligned}
$$

In the same way, it can be shown that

$$
\begin{aligned}
\Omega_{6}+ & \Omega_{8}+\Omega_{10}+\Omega_{13}+\Omega_{16}+\Omega_{18}+\Omega_{20}+\Omega_{22}+\Omega_{24}+\Omega_{26}+\Omega_{29}+\Omega_{32}+\Omega_{35} \\
= & \frac{C(n) Y}{\log Y \log N}\left(\int_{\frac{8}{33}}^{\frac{37}{150}} \frac{d t}{t} \int_{\frac{41}{90}-t}^{\frac{16}{33}-t} \frac{1}{u^{2}} w\left(\frac{1-t-u}{u}\right) d u\right. \\
& +\int_{\frac{37}{150}}^{\frac{31}{99}} \frac{d t}{t} \int_{\frac{701}{1566}-t}^{\frac{16}{33}-t} \frac{1}{u^{2}} w\left(\frac{1-t-u}{u}\right) d u \\
& +\int_{\frac{31}{99}}^{\frac{859}{2610}} \frac{d t}{t} \int_{\frac{89}{201}-\frac{58}{67} t}^{\frac{701}{1566}-t} \frac{1}{u^{2}} w\left(\frac{1-t-u}{u}\right) d u \\
& \left.+\int_{\frac{859}{2610}}^{\frac{10}{27}} \frac{d t}{\frac{89}{201}-\frac{58}{67} t} \frac{1}{u^{2}} w\left(\frac{1-t-u}{u}\right) d u+O(\delta)\right) \\
\geq & \frac{C(n) Y}{\log Y \log N}\left(\int_{\frac{8}{135}}^{\frac{37}{150}} \frac{d t}{t} \int_{\frac{41}{90}-t}^{\frac{16}{33}-t} \frac{1}{u(1-t-u)}\left(1+\log \left(\frac{1-t}{u}-2\right)\right) d u\right. \\
& +\int_{\frac{37}{150}}^{\frac{619}{2349}} \frac{d t}{t} \int_{\frac{701}{1566}-t}^{\frac{16}{33}-t} \frac{1}{u(1-t-u)}\left(1+\log \left(\frac{1-t}{u}-2\right)\right) d u \\
&
\end{aligned}
$$




$$
\begin{aligned}
& +0.5607 \int_{\frac{619}{2349}}^{\frac{31}{99}} \frac{d t}{t} \int_{\frac{701}{1566}-t}^{\frac{1}{4}(1-t)} \frac{d u}{u^{2}} \\
& +\int_{\frac{619}{2349}}^{\frac{31}{99}} \frac{d t}{t} \int_{\frac{1}{4}(1-t)}^{\frac{16}{33}-t} \frac{1}{u(1-t-u)}\left(1+\log \left(\frac{1-t}{u}-2\right)\right) d u \\
& +0.5607 \int_{\frac{31}{2610}}^{\frac{85}{99}} \frac{d t}{t} \int_{\frac{89}{201}-\frac{58}{67} t}^{\frac{701}{1566}-t} \frac{d u}{u^{2}} \\
& \left.+0.5607 \int_{\frac{859}{27}}^{\frac{10}{2610}} \frac{d t}{t} \int_{\frac{16}{201}}^{\frac{89}{201}-\frac{58}{67} t} \frac{d u}{u^{2}}+O(\delta)\right) \\
& +0.08530+0.031194+0.066046+0.050732+0.051408 \\
& =0.289310 \cdot \frac{C(n) Y}{\log Y \log N} .
\end{aligned}
$$

The proof of Lemma 26 is complete.

Lemma 27. We have

$$
\begin{aligned}
\Phi & =\Omega_{30}+\Omega_{33}+\Omega_{36}+\Omega_{38}+\Omega_{40}+\Omega_{42}+\Omega_{46}+\Omega_{49}+\Omega_{53}+\Omega_{66} \\
& \geq 0.024582 \cdot \frac{C(n) Y}{\log Y \log N} .
\end{aligned}
$$

Pr o of. On applying Lemmas 11, 12, 18, 20 and 21, in the same way as in Lemma 24, we have

$$
\begin{aligned}
\Phi= & \frac{C(n) Y}{\log Y \log N}\left(\int_{\frac{34}{99}}^{\frac{1649}{4752}} \frac{d t}{t} \int_{\frac{85}{99}-2 t}^{\frac{85}{147}-\frac{58}{49} t} \frac{1}{u^{2}} w\left(\frac{1-t-u}{u}\right) d u\right. \\
& +\int_{\frac{1649}{4752}}^{\frac{1823}{4725}} \frac{d t}{t} \int_{\frac{85}{147}-\frac{58}{49} t}^{\frac{34}{4^{2}}-\frac{70}{59} t} \frac{1}{u^{2}} w\left(\frac{1-t-u}{u}\right) d u \\
& +\int_{\frac{1823}{7830}}^{\frac{3041}{4725}} \frac{d t}{t} \int_{\frac{16}{147}}^{\frac{58}{49} t} \frac{1}{u^{2}} w\left(\frac{1-t-u}{u}\right) d u \\
& +\int_{\frac{11}{114}}^{\frac{49}{114}} \frac{d t}{t} \int_{\frac{89}{114}-\frac{29}{19} t}^{\frac{18}{23} t} \frac{1}{u^{2}} w\left(\frac{1-t-u}{u}\right) d u
\end{aligned}
$$




$$
\begin{aligned}
& +\int_{\frac{3397}{7830}}^{\frac{4}{9}} \frac{d t}{t} \int_{\frac{17}{12}-\frac{35}{12} t}^{\frac{17}{12}-\frac{29}{10} t} \frac{1}{u^{2}} w\left(\frac{1-t-u}{u}\right) d u \\
& \left.+\int_{\frac{211}{432}}^{\frac{281}{570}} \frac{d t}{t} \int_{\frac{89}{27}-\frac{58}{9} t}^{\frac{36}{11}-\frac{70}{11} t} \frac{1}{u^{2}} w\left(\frac{1-t-u}{u}\right) d u+O(\varepsilon)\right) \\
& \geq \frac{C(n) Y}{\log Y \log N}\left(\int_{\frac{34}{99}}^{\frac{1649}{4752}} \frac{d t}{t} \int_{\frac{85}{99}-2 t}^{\frac{85}{147}-\frac{58}{49} t} \frac{1}{u(1-t-u)}\left(1+\log \left(\frac{1-t}{u}-2\right)\right) d u\right. \\
& +\int_{\frac{1649}{4752}}^{\frac{77}{221}} \frac{d t}{t} \int_{\frac{34}{59}-\frac{70}{59} t}^{\frac{85}{147}-\frac{58}{49} t} \frac{1}{u(1-t-u)}\left(1+\log \left(\frac{1-t}{u}-2\right)\right) d u \\
& +0.5607 \int_{\frac{77}{221}}^{\frac{193}{549}} \frac{d t}{t} \int_{\frac{34}{59}-\frac{70}{59} t}^{\frac{1}{4}(1-t)} \frac{d u}{u^{2}} \\
& +\int_{\frac{77}{221}}^{\frac{193}{549}} \frac{d t}{t} \int_{\frac{1}{4}(1-t)}^{\frac{85}{147}-\frac{58}{49} t} \frac{1}{u(1-t-u)}\left(1+\log \left(\frac{1-t}{u}-2\right)\right) d u \\
& +0.5607 \int_{\frac{193}{549}}^{\frac{1823}{4725}} \frac{d t}{t} \int_{\frac{34}{59}-\frac{70}{59} t}^{\frac{85}{147}-\frac{58}{49} t} \frac{d u}{u^{2}}+0.5607 \int_{\frac{1823}{4725}}^{\frac{3041}{7830}} \frac{d t}{t} \int_{\frac{16}{135}}^{\frac{85}{147}-\frac{58}{49} t} \frac{d u}{u^{2}} \\
& +\int_{\frac{11}{27}}^{\frac{121}{291}} \frac{d t}{t} \int_{\frac{89}{114}-\frac{29}{19} t}^{\frac{18}{23}-\frac{35}{23} t} \frac{1}{u(1-t-u)}\left(1+\log \left(\frac{1-t}{u}-2\right)\right) d u \\
& +0.5607 \int_{\frac{121}{291}}^{\frac{49}{117}} \frac{d t}{t} \int_{\frac{89}{114}-\frac{29}{19} t}^{\frac{1}{4}(1-t)} \frac{d u}{u^{2}} \\
& +\int_{\frac{121}{291}}^{\frac{49}{117}} \frac{d t}{t} \int_{\frac{1}{4}(1-t)}^{\frac{18}{23}-\frac{35}{23} t} \frac{1}{u(1-t-u)}\left(1+\log \left(\frac{1-t}{u}-2\right)\right) d u \\
& +0.5607 \int_{\frac{49}{117}}^{\frac{49}{114}} \frac{d t}{t} \int_{\frac{89}{114}-\frac{29}{19} t}^{\frac{18}{23}-\frac{35}{23} t} \frac{d u}{u^{2}} \\
& +\int_{\frac{3397}{7830}}^{\frac{7}{16}} \frac{d t}{t} \int_{\frac{17}{12}-\frac{35}{12} t}^{\frac{17}{12}-\frac{29}{10} t} \frac{1}{u(1-t-u)}\left(1+\log \left(\frac{1-t}{u}-2\right)\right) d u
\end{aligned}
$$




$$
\begin{aligned}
& +0.5607 \int_{\frac{7}{16}}^{\frac{70}{159}} \frac{d t}{t} \int_{\frac{17}{12}-\frac{35}{12} t}^{\frac{1}{4}(1-t)} \frac{d u}{u^{2}} \\
& +\int_{\frac{7}{16}}^{\frac{70}{159}} \frac{d t}{t} \int_{\frac{1}{4}(1-t)}^{\frac{17}{12}-\frac{29}{10} t} \frac{1}{u(1-t-u)}\left(1+\log \left(\frac{1-t}{u}-2\right)\right) d u \\
& +0.5607 \int_{\frac{70}{159}}^{\frac{4}{9}} \frac{d t}{t} \int_{\frac{17}{12}-\frac{35}{12} t}^{\frac{17}{12}-\frac{29}{10} t} \frac{d u}{u^{2}} \\
& +\int_{\frac{211}{432}}^{\frac{329}{669}} \frac{d t}{t} \int_{\frac{89}{27}-\frac{58}{9} t}^{\frac{36}{11}-\frac{70}{11} t} \frac{1}{u(1-t-u)}\left(1+\log \left(\frac{1-t}{u}-2\right)\right) d u \\
& +0.5607 \int_{\frac{329}{669}}^{\frac{281}{570}} \frac{d t}{t} \int_{\frac{89}{27}-\frac{58}{9} t}^{\frac{1}{4}(1-t)} \frac{d u}{u^{2}} \\
& \left.+\int_{\frac{329}{669}}^{\frac{281}{570}} \frac{d t}{t} \int_{\frac{1}{4}(1-t)}^{\frac{36}{11}-\frac{70}{11} t} \frac{1}{u(1-t-u)}\left(1+\log \left(\frac{1-t}{u}-2\right)\right) d u+O(\varepsilon)\right) \\
& \geq(0.000303+0.000244+0.000282+0.000276+0.008036 \\
& +0.000393+0.001843+0.000369+0.000357+0.003087 \\
& +0.001545+0.000679+0.000634+0.002319+0.002910 \\
& +0.000330+0.000975) \cdot \frac{C(n) Y}{\log Y \log N} \\
& =0.024582 \cdot \frac{C(n) Y}{\log Y \log N} \text {. }
\end{aligned}
$$

The proof of Lemma 27 is complete.

Lemma 28. We have

$$
\begin{aligned}
\Phi & =\Omega_{1}+\Omega_{2}+\Omega_{3}+\Omega_{5}+\Omega_{7}+\Omega_{9}+\Omega_{12}+\Omega_{15} \\
& \geq 1.010623 \cdot \frac{C(n) Y}{\log Y \log N} .
\end{aligned}
$$

Pr o of. The main idea in this lemma is adopted from [21]. We have

$$
\begin{aligned}
\Omega_{1} & \geq \sum_{Y^{\frac{16}{135}}<p \leq Y^{\frac{89}{462}}-10^{-8}} \sum_{Y^{\frac{16}{135}<q<p}} S\left(\mathcal{A}_{p q}, q\right) \\
& =\sum_{Y^{\frac{16}{135}}<p \leq Y^{\frac{89}{462}-10^{-8}}} \sum_{Y^{\frac{16}{135}<q<p}} S\left(\mathcal{A}_{p q}, Y^{\delta}\right)
\end{aligned}
$$




$$
\begin{aligned}
& -\sum_{Y^{\frac{16}{135}}<p \leq Y^{\frac{89}{462}-10^{-8}}} \sum_{Y^{\frac{16}{135}<q<p}} \sum_{Y^{\delta}<r<Y^{\frac{16}{135}}} S\left(\mathcal{A}_{p q r}, r\right) \\
& -\sum_{Y^{\frac{16}{135}}<p \leq Y^{\frac{89}{462}}-10^{-8}} \sum_{Y^{\frac{16}{135}<q<p}} \sum_{Y^{\frac{16}{135}}<r<\min \left(q,\left(\frac{2 Y}{p q}\right)^{\frac{1}{2}}\right)} S\left(\mathcal{A}_{p q r}, r\right) \\
& =\Phi_{1}-\Phi_{2}-\Phi_{3} \text {. }
\end{aligned}
$$

Let $z=Y^{\delta}$ and $D(p, q)=Y^{\frac{19}{36}} /(p q)$. Using Iwaniec's sieve method, in the same way as in Lemma 26, we have

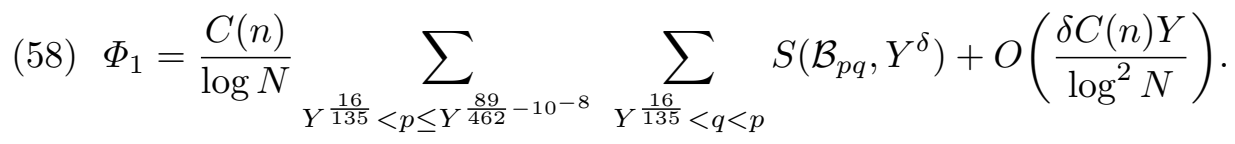

Lemma 19 yields

(59) $\Phi_{2}$

$$
=\frac{C(n)}{\log N} \sum_{Y^{\frac{16}{135}<p \leq Y \frac{89}{462}-10^{-8}}} \sum_{Y^{\frac{16}{135}<q<p}} \sum_{Y^{\delta}<r<Y^{\frac{16}{135}}} S\left(\mathcal{B}_{p q r}, r\right)+O\left(\frac{\delta C(n) Y}{\log ^{2} N}\right) .
$$

Hence,

$$
\begin{aligned}
(60) \Phi_{1}- & \Phi_{2} \\
= & \frac{C(n)}{\log N} \sum_{Y \frac{16}{135}<p \leq Y \frac{89}{462}-10^{-8}} \sum_{Y Y^{\frac{16}{135}<q<p}} S\left(\mathcal{B}_{p q}, Y^{\frac{16}{135}}\right) \\
& +O\left(\frac{\delta C(n) Y}{\log ^{2} N}\right) \\
= & \frac{C(n) Y}{\log Y \log N} \cdot \frac{135}{16} \int_{\frac{16}{135}}^{\frac{89}{462}-10^{-8}} \frac{d t}{t} \int_{\frac{16}{135}}^{t} \frac{1}{u} w\left(\frac{135}{16}(1-t-u)\right) d u \\
& \left.+O\left(\frac{\delta C(n) Y}{\log 2}\right){ }^{2}\right) \\
\geq & \frac{C(n) Y}{\log Y \log N}\left(\frac{135}{16} \int_{\frac{16}{135}}^{\frac{89}{462}} \frac{d t}{t} \int_{\frac{16}{135}}^{t} \frac{1}{u} w\left(\frac{135}{16}(1-t-u)\right) d u-10^{-6}\right) \\
\geq & 0.558649 \cdot \frac{C(n) Y}{\log Y \log N} .
\end{aligned}
$$


Note that if $q<(2 Y / p)^{\frac{1}{3}}$, then $q<(2 Y /(p q))^{\frac{1}{2}}$. We have

$$
\begin{aligned}
(61) \Phi_{3} \leq & \sum_{Y^{\frac{16}{135}}<p \leq Y^{\frac{89}{462}-10^{-8}}} \sum_{Y^{\frac{16}{135}<q<p}} \sum_{Y^{\frac{16}{135}<r<q}} S\left(\mathcal{A}_{p q r}, Y^{\frac{16}{135}}\right) \\
= & \sum_{Y^{\frac{16}{135}}<p \leq Y^{\frac{89}{462}-10^{-8}}} \sum_{Y^{\frac{16}{135}<q<p}} \sum_{Y^{\frac{16}{135}<r<q}} S\left(\mathcal{A}_{p q r}, Y^{\delta}\right) \\
& -\sum_{Y^{\frac{16}{135}}<p \leq Y^{\frac{89}{462}-10^{-8}}} \sum_{Y^{\frac{16}{135}<q<p}} \sum_{Y^{\frac{16}{135}<r<q}} \sum_{Y^{\delta}<s<Y^{\frac{16}{135}}} S\left(\mathcal{A}_{p q r s}, s\right) \\
= & \Phi_{4}-\Phi_{5} .
\end{aligned}
$$

Let $z=Y^{\delta}$ and $D(p, q, r)=Y^{10^{-8}}$. Using Iwaniec's sieve method and Lemma 16, in the same way as in Lemma 26, we have

$$
\begin{aligned}
(62) \Phi_{4}= & \frac{C(n)}{\log N} \sum_{Y^{\frac{16}{135}}<p \leq Y}^{\frac{89}{462}-10^{-8}} \sum_{Y^{\frac{16}{135}<q<p}} \sum_{Y^{\frac{16}{135}<r<q}} S\left(\mathcal{B}_{p q r}, Y^{\delta}\right) \\
& +O\left(\frac{\delta C(n) Y}{\log ^{2} N}\right) .
\end{aligned}
$$

Lemma 19 yields

(63) $\Phi_{5}$

$$
\begin{aligned}
= & \frac{C(n)}{\log N} \sum_{Y^{\frac{16}{135}}<p \leq Y^{\frac{89}{462}-10^{-8}}} \sum_{Y^{\frac{16}{135}<q<p}} \sum_{Y^{\frac{16}{135}<r<q}} \sum_{Y^{\delta}<s<Y^{\frac{16}{135}}} S\left(\mathcal{B}_{\text {pqrs }}, s\right) \\
& +O\left(\frac{\delta C(n) Y}{\log ^{2} N}\right) .
\end{aligned}
$$

We therefore have

$$
\begin{aligned}
\Phi_{3} \leq & \frac{C(n)}{\log N} \sum_{Y \frac{16}{135}<p \leq Y} \sum_{\frac{89}{462}-10^{-8}} \sum_{Y\left(\frac{16}{135}<q<p\right.} \sum_{Y(n) Y} S\left(\mathcal{B}_{p q r}, Y^{\frac{16}{135}}\right) \\
& +O r<q \\
\leq & \frac{C(n) Y}{\log Y \log N} \cdot \frac{135}{16} \int_{\frac{16}{135}}^{\frac{89}{462}} \frac{d t}{t} \int_{\frac{16}{135}}^{t} \frac{d u}{u} \int_{\frac{16}{135}}^{u} \frac{1}{v} w\left(\frac{135}{16}(1-t-u-v)\right) d v \\
\leq & \frac{C(n) Y}{\log Y \log N}\left(0.5617 \cdot \frac{135}{16} \int_{\frac{16}{135}}^{\frac{71}{405}} \frac{d t}{t} \int_{\frac{16}{135}}^{t} \frac{d u}{u} \int_{\frac{16}{135}}^{u} \frac{d v}{v}\right.
\end{aligned}
$$




$$
\begin{aligned}
& +0.5617 \cdot \frac{135}{16} \int_{\frac{71}{405}}^{\frac{89}{462}} \frac{d t}{t} \int_{\frac{16}{135}}^{\frac{1}{2}\left(\frac{71}{135}-t\right)} \frac{d u}{u} \int_{\frac{16}{135}}^{u} \frac{d v}{v} \\
& \left.+0.5644 \cdot \frac{135}{16} \int_{\frac{71}{405}}^{\frac{89}{462}} \frac{d t}{t} \int_{\frac{1}{2}\left(\frac{71}{135}-t\right)}^{t} \frac{d u}{u} \int_{\frac{16}{135}}^{u} \frac{d v}{v}\right) \\
\leq & (0.047392+0.030099+0.013113) \cdot \frac{C(n) Y}{\log Y \log N} \\
= & 0.090604 \cdot \frac{C(n) Y}{\log Y \log N} .
\end{aligned}
$$

Hence,

$$
\Omega_{1} \geq 0.468045 \cdot \frac{C(n) Y}{\log Y \log N} .
$$

In the same way, it can be shown that

$$
\begin{aligned}
& \Omega_{2}+\Omega_{3}+\Omega_{5}+\Omega_{7}+\Omega_{9}+\Omega_{12}+\Omega_{15} \\
& \geq \frac{C(n) Y}{\log Y \log N}\left(\frac{135}{16} \int_{\frac{89}{462}}^{\frac{17}{54}} \frac{d t}{t} \int_{\frac{16}{135}}^{\frac{89}{288}-\frac{29}{48} t} \frac{1}{u} w\left(\frac{135}{16}(1-t-u)\right) d u\right. \\
& \left.-\frac{135}{16} \int_{\frac{89}{462}}^{\frac{17}{54}} \frac{d t}{t} \int_{\frac{16}{135}}^{\frac{89}{288}-\frac{29}{48} t} \frac{d u}{u} \int_{\frac{16}{135}}^{u} \frac{1}{v} w\left(\frac{135}{16}(1-t-u-v)\right) d v-10^{-6}\right) \\
& \geq \frac{C(n) Y}{\log Y \log N}\left(0.5612 \cdot \frac{135}{16} \int_{\frac{89}{462}}^{\frac{17}{54}} \frac{d t}{t} \int_{\frac{16}{135}}^{\frac{89}{288}-\frac{29}{48} t} \frac{d u}{u}\right. \\
& -0.5617 \cdot \frac{135}{16} \int_{\frac{89}{462}}^{\frac{13}{45}} \frac{d t}{t} \int_{\frac{16}{135}}^{\frac{1}{2}\left(\frac{71}{135}-t\right)} \frac{d u}{u} \int_{\frac{16}{135}}^{u} \frac{d v}{v} \\
& -0.5644 \cdot \frac{135}{16} \int_{\frac{89}{462}}^{\frac{13}{45}} \frac{d t}{t} \int_{\frac{1}{2}\left(\frac{71}{135}-t\right)}^{\frac{89}{288}-\frac{29}{48} t} \frac{d u}{u} \int_{\frac{16}{135}}^{u} \frac{d v}{v} \\
& \left.-0.5644 \cdot \frac{135}{16} \int_{\frac{13}{45}}^{\frac{17}{54}} \frac{d t}{t} \int_{\frac{16}{135}}^{\frac{89}{288}-\frac{29}{48} t} \frac{d u}{u} \int_{\frac{16}{135}}^{u} \frac{d v}{v}-10^{-6}\right) \\
& \geq(0.658048-0.044320-0.069979-0.001171) \cdot \frac{C(n) Y}{\log Y \log N} \\
& =0.542578 \cdot \frac{C(n) Y}{\log Y \log N} .
\end{aligned}
$$


Hence,

$$
\Phi \geq 1.010623 \cdot \frac{C(n) Y}{\log Y \log N} .
$$

The proof of Lemma 28 is complete.

11. Proof of the Theorem. From Lemmas 24-28, it follows that

$$
\Omega \geq 2.104057 \cdot \frac{C(n) Y}{\log Y \log N} .
$$

Then using Lemmas 22 and 23, we obtain

$$
T(n)=S\left(\mathcal{A},(2 Y)^{\frac{1}{2}}\right)+O\left(Y^{\frac{1}{2}}\right) \geq 0.011 \cdot \frac{C(n) Y}{\log Y \log N} .
$$

Now (44) holds and the proof of the Theorem is complete.

\section{References}

[1] P. X. Gallagher, A large sieve density estimate near $\sigma=1$, Invent. Math. 11 (1970), 329-339.

[2] D. R. Heath-Brown and H. Iwaniec, On the difference between consecutive primes, ibid. 55 (1979), 49-69.

[3] L. K. Hua, Some results in the additive prime number theory, Quart. J. Math. Oxford 9 (1938), 68-80.

[4] M. N. Huxley, On the difference between consecutive primes, Invent. Math. 15 (1972), 164-170.

[5] -, Large values of Dirichlet polynomials III, Acta Arith. 26 (1975), 435-444.

[6] H. Iwaniec, A new form of the error term in the linear sieve, ibid. 37 (1980), $307-320$

[7] C. H. Jia, Three primes theorem in a short interval (III), Sci. China Ser. A 34 (1991), 1039-1056.

[8] —, Three primes theorem in a short interval (V), Acta Math. Sinica (N.S.) 7 (1991), 135-170.

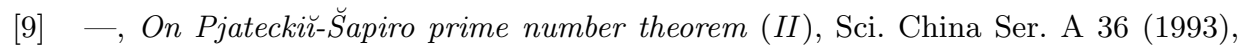
913-926.

[10] - , Goldbach numbers in short interval, Sci. China Ser. A 24 (1994), 1233-1259 (in Chinese); I. Sci. China Ser. A 38 (1995), 385-406; II. Sci. China Ser. A 38 (1995), $513-523$.

[11] - On the difference between consecutive primes, Sci. China Ser. A 38 (1995), $1163-1186$.

[12] H. Li, On the Goldbach numbers in short interval, Sci. China Ser. A, to appear.

[13] H. Mikawa, On the exceptional set in Goldbach's problem, Tsukuba J. Math. 16 (1992), 513-543.

[14] H. L. Montgomery, Topics in Multiplicative Number Theory, Lecture Notes in Math. 227, Springer, Berlin, 1971.

[15] Chengdong P an and Chengbiao P an, Goldbach Conjecture, Science Press, Beijing, 1981 (in Chinese). 
[16] Chengdong Pan and Chengbiao Pan, The Basis of Analytic Number Theory, Science Press, Beijing, 1991 (in Chinese).

[17] A. Perelli and J. Pintz, On the exceptional set for Goldbach's problem in short intervals, J. London Math. Soc. (2) 47 (1993), 41-49.

[18] K. Ramachandra, On the number of Goldbach numbers in small intervals, J. Indian Math. Soc. 37 (1973), 157-170.

[19] B. Saffari and R. C. Vaughan, On the fractional parts of $x / n$ and related sequences II, Ann. Inst. Fourier (Grenoble) 27 (1977), 1-30.

[20] P. Shiu, A Brun-Titchmarsh theorem for multiplicative functions, J. Reine Angew. Math. 313 (1980), 161-170.

[21] N. Watt, Short intervals almost all containing primes, Acta Arith. 72 (1995), 131167.

Institute of Mathematics

Academia Sinica

Beijing, 100080, China 